\title{
Black Holes in Higher Dimensions
}

\author{
Roberto Emparan \\ Institució Catalana de Recerca i Estudis Avançats (ICREA) \\ and \\ Departament de Física Fonamental, Universitat de Barcelona \\ Marti i Franquès 1, E-08028 Barcelona, Spain \\ email: emparan@ub.edu \\ Harvey S. Reall \\ Department of Applied Mathematics and Theoretical Physics \\ University of Cambridge, Centre for Mathematical Sciences \\ Wilberforce Road, Cambridge CB3 0WA \\ United Kingdom \\ email: hsr1000@cam.ac.uk
}

\section{Living Reviews in Relativity \\ ISSN 1433-8351}

Accepted on 7 July 2008

Published on 8 September 2008

\begin{abstract}
We review black-hole solutions of higher-dimensional vacuum gravity and higher-dimensional supergravity theories. The discussion of vacuum gravity is pedagogical, with detailed reviews of Myers-Perry solutions, black rings, and solution-generating techniques. We discuss blackhole solutions of maximal supergravity theories, including black holes in anti-de Sitter space. General results and open problems are discussed throughout.
\end{abstract}

This review is licensed under a Creative Commons Attribution-Non-Commercial-NoDerivs 2.0 Germany License. http://creativecommons.org/licenses/by-nc-nd/2.0/de/ 


\section{Imprint / Terms of Use}

Living Reviews in Relativity is a peer reviewed open access journal published by the Max Planck Institute for Gravitational Physics, Am Mühlenberg 1, 14476 Potsdam, Germany. ISSN 1433-8351.

This review is licensed under a Creative Commons Attribution-Non-Commercial-NoDerivs 2.0

Germany License: http://creativecommons.org/licenses/by-nc-nd/2.0/de/

Because a Living Reviews article can evolve over time, we recommend to cite the article as follows:

Roberto Emparan and Harvey S. Reall,

"Black Holes in Higher Dimensions",

Living Rev. Relativity, 11, (2008), 6. [Online Article]: cited [<date $>$ ],

http://www.livingreviews.org/lrr-2008-6

The date given as $<$ date $>$ then uniquely identifies the version of the article you are referring to.

\section{Article Revisions}

Living Reviews supports two different ways to keep its articles up-to-date:

Fast-track revision A fast-track revision provides the author with the opportunity to add short notices of current research results, trends and developments, or important publications to the article. A fast-track revision is refereed by the responsible subject editor. If an article has undergone a fast-track revision, a summary of changes will be listed here.

Major update A major update will include substantial changes and additions and is subject to full external refereeing. It is published with a new publication number.

For detailed documentation of an article's evolution, please refer always to the history document of the article's online version at http://www.livingreviews.org/lrr-2008-6. 


\section{Contents}

1 Introduction $\quad \mathbf{5}$

1.1 Why gravity is richer in $d>4 \ldots \ldots \ldots \ldots \ldots$

1.2 Why gravity is more difficult in $d>4 \ldots \ldots \ldots \ldots$. . . . . . . . . . . . . . . . . . . . . .

2 Scope and Organization of this Article $\quad 9$

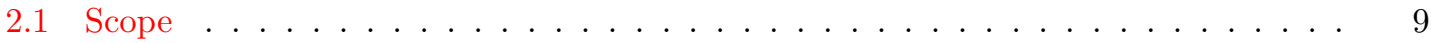

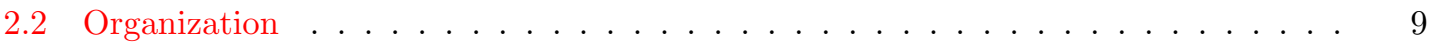

3 Basic Concepts and Solutions $\quad 10$

3.1 Conserved charges . . . . . . . . . . . . . . . . . . 10

3.2 The Schwarzschild-Tangherlini solution and black $p$-branes . . . . . . . . . . . 12

3.3 Stability of the static black hole . . . . . . . . . . . . . . . . . . 13

3.4 Gregory-Laflamme instability . . . . . . . . . . . . . . . . . . 13

4 Myers-Perry Solutions $\quad 15$

4.1 Rotation in a single plane . . . . . . . . . . . . . . . . . . . . . 15

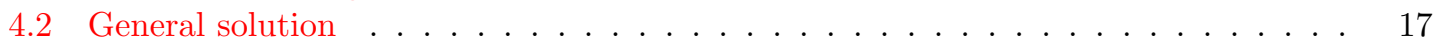

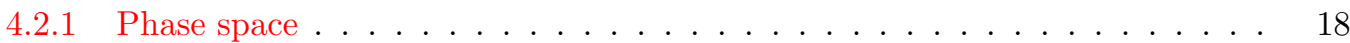

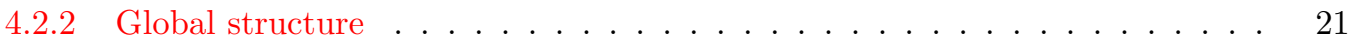

4.3 Symmetries . . . . . . . . . . . . . . . . . . . . . . . . 21

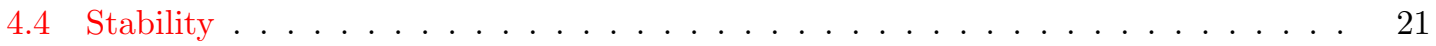

5 Vacuum Solutions in Five Dimensions 2

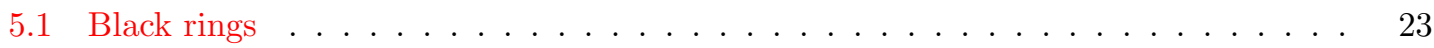

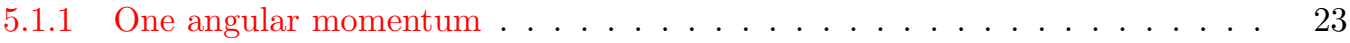

5.1 .2 Two angular momenta . . . . . . . . . . . . . . 26

5.2 Stationary axisymmetric solutions with $d-3$ rotational symmetries . . . . . . 30

5.2 .1 Weyl solutions . . . . . . . . . . . . . . . . . . . . . . . . . . .

5.2 .2 General axisymmetric class . . . . . . . . . . . . . . . . . . . . . . . . . . . . 32

5.3 Multiple-black-hole solutions . . . . . . . . . . . . . . . . . . . 39

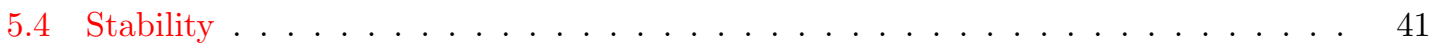

6 Vacuum Solutions in More Than Five Dimensions $\quad 43$

6.1 Approximate solutions from curved thin branes . . . . . . . . . . . . . . . . . . . . 43

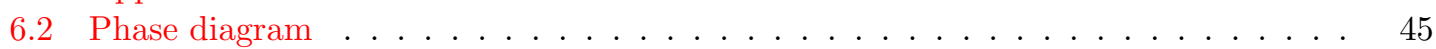

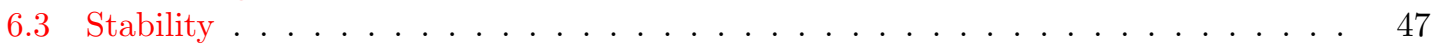

7 Solutions with a Gauge Field $\quad 48$

7.1 Introduction . . . . . . . . . . . . . . . . . . . . . 48

7.2 Topologically-spherical black holes . . . . . . . . . . . . . . . . . . . . . . 48

7.2 .1 Nonextremal solutions . . . . . . . . . . . . . . . . . . . . 48

7.2.2 Supersymmetric solutions: the Breckenridge-Myers-Peet-Vafa (BMPV) black

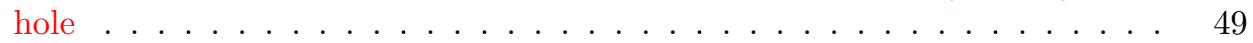

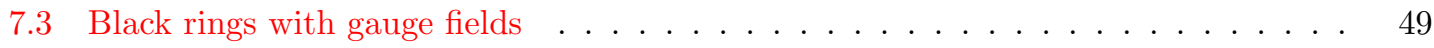

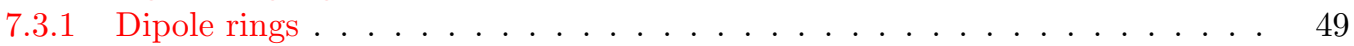

7.3.2 Charged black rings, supersymmetric black rings . . . . . . . . . . . . 49

7.4 Solution-generating techniques . . . . . . . . . . . . . . . . . . . . . . . . . . . . . .

7.5 Multiple-black-hole solutions . . . . . . . . . . . . . . . . . . . 51 
8 General Results and Open Problems $\quad 52$

8.1 Introduction . . . . . . . . . . . . . . . . . . . . 52

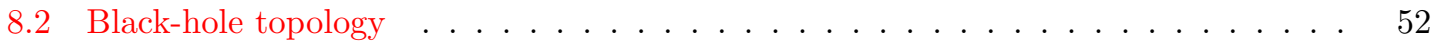

8.3 Uniqueness of static black holes . . . . . . . . . . . . . . . . . . . . . . . . . . . . . . . . . . . . . . . . . . .

8.4 Stationary black holes . . . . . . . . . . . . . . . . . . . . . . . . . . . . . . . . . . . . . . . . .

8.5 Supersymmetric black holes . . . . . . . . . . . . . . . . . . 54

8.6 Algebraic classification . . . . . . . . . . . . . . . . . . . . 55

8.7 Laws of black-hole mechanics . . . . . . . . . . . . . . . . . . . 56

8.8 Hawking radiation and black-hole thermodynamics . . . . . . . . . . . . . . 56

8.9 Apparent and isolated horizons and critical phenomena . . . . . . . . . . . 57

9 Solutions with a Cosmological Constant $\quad 59$

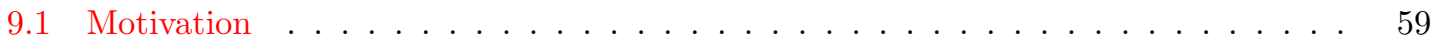

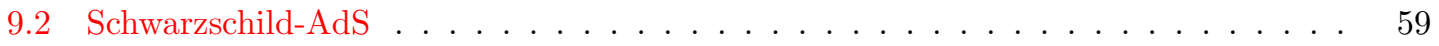

9.3 Stationary vacuum solutions . . . . . . . . . . . . . . . . . . . . . 60

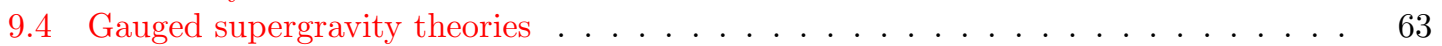

9.5 Static charged solutions . . . . . . . . . . . . . . . . . . . . . 63

9.6 Stationary charged solutions . . . . . . . . . . . . . . . . . . . 64

10 Acknowledgments $\quad 67$

$\begin{array}{lr}\text { References } & 68\end{array}$ 


\section{Introduction}

Classical general relativity in more than four spacetime dimensions has been the subject of increasing attention in recent years. Among the reasons it should be interesting to study this extension of Einstein's theory, and in particular its black-hole solutions, we may mention that

- String theory contains gravity and requires more than four dimensions. In fact, the first successful statistical counting of black-hole entropy in string theory was performed for a fivedimensional black hole [229]. This example provides the best laboratory for the microscopic string theory of black holes.

- The AdS/CFT correspondence relates the dynamics of a $d$-dimensional black hole with those of a quantum field theory in $d-1$ dimensions [187] (for a review see [1]).

- The production of higher-dimensional black holes in future colliders becomes a conceivable possibility in scenarios involving large extra dimensions and TeV-scale gravity [30, 155].

- As mathematical objects, black-hole spacetimes are among the most important Lorentzian Ricci-flat manifolds in any dimension.

These, however, refer to applications of the subject - important though they are - but we believe that higher-dimensional gravity is also of intrinsic interest. Just as the study of quantum field theories, with a field content very different than any conceivable extension of the Standard Model, has been a very useful endeavor, throwing light on general features of quantum fields, we believe that endowing general relativity with a tunable parameter - namely the spacetime dimensionality $d$-should also lead to valuable insights into the nature of the theory, in particular into its most basic objects: black holes. For instance, four-dimensional black holes are known to have a number of remarkable features, such as uniqueness, spherical topology, dynamical stability, and to satisfy a set of simple laws - the laws of black hole mechanics. One would like to know which of these are peculiar to four-dimensions, and which hold more generally. At the very least, this study will lead to a deeper understanding of classical black holes and of what spacetime can do at its most extreme.

There is a growing awareness that the physics of higher-dimensional black holes can be markedly different, and much richer, than in four dimensions. Arguably, two advances are largely responsible for this perception: the discovery of dynamical instabilities in extended black-hole horizons [118] and the discovery of black-hole solutions with horizons of nonspherical topology that are not fully characterized by their conserved charges [83].

At the risk of anticipating results and concepts that will be developed only later in this review, in the following we try to give simple answers to two frequently asked questions: 1) why should one expect any interesting new dynamics in higher-dimensional general relativity, and 2) what are the main obstacles to a direct generalization of the four-dimensional techniques and results. A straightforward answer to both questions is to simply say that as the number of dimensions grows, the number of degrees of freedom of the gravitational field also increases, but more specific, yet intuitive, answers are possible.

\subsection{Why gravity is richer in $d>4$}

The novel features of higher-dimensional black holes that have been identified so far can be understood in physical terms as due to the combination of two main ingredients: different rotation dynamics and the appearance of extended black objects.

There are two aspects of rotation that change significantly when spacetime has more than four dimensions. First, there is the possibility of rotation in several independent rotation planes [200]. 
The rotation group $S O(d-1)$ has Cartan subgroup $U(1)^{N}$, with

$$
N \equiv\left\lfloor\frac{d-1}{2}\right\rfloor ;
$$

hence, there is the possibility of $N$ independent angular momenta. In simpler and more explicit terms, one can group the $d-1$ spatial dimensions (say, at asymptotically-flat infinity) into pairs $\left(x_{1}, x_{2}\right),\left(x_{3}, x_{4}\right), \ldots$, each pair defining a plane, and choose polar coordinates in each plane, $\left(r_{1}, \phi_{1}\right)$, $\left(r_{2}, \phi_{2}\right), \ldots$ Here we see the possibility of having $N$ independent (commuting) rotations associated to the vectors $\partial_{\phi_{1}}, \partial_{\phi_{2}} \ldots$. To each of these rotations we associate an angular momentum component $J_{i}$.

The other aspect of rotation that changes qualitatively as the number of dimensions increases is the relative competition between the gravitational and centrifugal potentials. The radial falloff of the Newtonian potential

$$
-\frac{G M}{r^{d-3}}
$$

depends on the number of dimensions, whereas the centrifugal barrier

$$
\frac{J^{2}}{M^{2} r^{2}}
$$

does not, since rotation is confined to a plane. We see that the competition between (2) and (3) is different in $d=4, d=5$, and $d \geq 6$. In Newtonian physics this is well known to result in a different stability of Keplerian orbits, but this precise effect is not directly relevant to the blackhole dynamics we are interested in. Still, the same kind of dimension dependence will have rather dramatic consequences for the behavior of black holes.

The other novel ingredient that appears in $d>4$ but is absent in lower dimensions (at least in vacuum gravity) is the presence of black objects with extended horizons, i.e., black strings and, in general, black $p$-branes. Although these are not asymptotically-flat solutions, they provide the basic intuition for understanding novel kinds of asymptotically-flat black holes.

Let us begin with the simple observation that, given a black-hole solution of the vacuum Einstein equations in $d$ dimensions with horizon geometry $\Sigma_{H}$, we can immediately construct a vacuum solution in $d+1$ dimensions by simply adding a flat spatial direction ${ }^{1}$. The new horizon geometry is then a black string with horizon $\Sigma_{H} \times \mathbb{R}$. Since the Schwarzschild solution is easily generalized to any $d \geq 4$, it follows that black strings exist in any $d \geq 5$. In general, adding $p$ flat directions we find that black $p$-branes with horizon $S^{q} \times \mathbb{R}^{p}$ (with $q \geq 2$ ) exist in any $d \geq 6+p-q$.

How are these related to new kinds of asymptotically-flat black holes? Heuristically, take a piece of black string with $S^{q} \times \mathbb{R}$ horizon, and curve it to form a black ring with horizon topology $S^{q} \times S^{1}$. Since the black string has a tension, the $S^{1}$, being contractible, will tend to collapse. But we may try to set the ring into rotation and in this way provide a centrifugal repulsion that balances the tension. This turns out to be possible in any $d \geq 5$, so we expect that nonspherical horizon topologies are a generic feature of higher-dimensional general relativity.

It is also natural to try to apply this heuristic construction to black $p$-branes with $p>1$, namely, to bend the worldvolume spatial directions into a compact manifold and balance the tension by introducing suitable rotations. The possibilities are still under investigation, but it is clear that an increasing variety of black holes should be expected as $d$ grows. Observe again that the underlying reason is a combination of extended horizons with rotation.

Horizon topologies other than spherical are forbidden in $d=4$ by well-known theorems [132]. These are rigorous, but also rather technical and formal results. Can we find a simple, intuitive

\footnotetext{
${ }^{1}$ This is no longer true if the field equations involve not only the Ricci tensor but also the Weyl tensor, such as in Lovelock theories.
}

Living Reviews in Relativity

http: //www . livingreviews . org/lrr-2008-6 
explanation for the absence of vacuum black rings in $d=4$ ? The previous argument would trace this fact back to the absence of asymptotically-flat vacuum black holes in $d=3$. This is often attributed to the absence of propagating degrees of freedom for the three-dimensional graviton (or one of its paraphrases: $2+1$-gravity is topological, the Weyl tensor vanishes identically, etc), but here we shall use the simple observation that the quantity $G M$ is dimensionless in $d=3$. Hence, given any amount of mass, there is no length scale to tell us where the black-hole horizon should $\mathrm{be}^{2}$. So we attribute the absence of black strings in $d=4$ to the lack of such a scale. This observation goes some way towards understanding the absence of vacuum black rings with horizon topology $S^{1} \times S^{1}$ in four dimensions; it implies that there cannot exist black-ring solutions with different scales for each of the two circles, and, in particular one can not make one radius arbitrarily larger than the other. This argument, though, could still allow for black rings, where the radii of the two $S^{1}$ are set by the same scale, i.e., the black rings should be plump. The horizon-topology theorems then tell us that plump black rings do not exist; they would actually be within a spherical horizon.

Extended horizons also introduce a feature absent in $d=4$ : dynamical horizon instabilities [118]. Again, this is to some extent an issue of scales. Black brane horizons can be much larger in some of their directions than in others, and so perturbations with wavelengths on the order of the 'short' horizon length can fit several times along the 'long' extended directions. Since the horizon area tends to increase by dividing up the extended horizon into black holes of roughly the same size in all its dimensions, this provides grounds to expect an instability of the extended horizon (however, when other scales are present, as in charged solutions, the situation can become quite a bit more complicated). It turns out that higher-dimensional rotation can extend the horizon much more in some directions than in others, which is expected to trigger this kind of instability [81]. At the threshold of the instability, a zero-mode deformation of the horizon has been conjectured to lead to new 'pinched' black holes that do not have four-dimensional counterparts.

Finally, an important question raised in higher dimensions refers to the rigidity of the horizon. In four dimensions, stationarity implies the existence of a $U(1)$ rotational isometry [132]. In higher dimensions stationarity has been proven to imply one rigid rotation symmetry too [138], but not (yet?) more than one. However, all known higher-dimensional black holes have multiple rotational symmetries. Are there stationary black holes with less symmetry, for example just the single $U(1)$ isometry guaranteed in general? Or are black holes always as rigid as can be? This is, in our opinion, the main unsolved problem on the way to a complete classification of five-dimensional black holes and an important issue in understanding the possibilities for black holes in higher dimensions.

\subsection{Why gravity is more difficult in $d>4$}

Again, the simple answer to this question is the larger number of degrees of freedom. However, this cannot be an entirely satisfactory reply, since one often restricts oneself to solutions with a large degree of symmetry for which the number of actual degrees of freedom may not depend on the dimensionality of spacetime. A more satisfying answer should explain why the methods that are so successful in $d=4$ become harder, less useful, or even inapplicable, in higher dimensions.

Still, the larger number of metric components, and of equations determining them, is the main reason for the failure so far to find a useful extension of the Newman-Penrose (NP) formalism to $d>4$. This formalism, in which all the Einstein equations and Bianchi identities are written out explicitly, was instrumental in deriving the Kerr solution and analyzing its perturbations. The formalism is tailored to deal with algebraically-special solutions, but even if algebraic classifications have been developed for higher dimensions [51] and applied to known black-hole solutions, no

\footnotetext{
${ }^{2}$ It follows that the introduction of a length scale, for instance in the form of a (negative) cosmological constant, is a necessary condition for the existence of a black hole in $2+1$ dimensions. But gravity may still remain topological.
} 
practical extension of the NP formalism has appeared yet that can be used to derive the solutions, nor to study their perturbations.

Then, it seems natural to restrict oneself to solutions with a high degree of symmetry. Spherical symmetry yields easily by force of Birkhoff's theorem. The next simplest possibility is to impose stationarity and axial symmetry. In four dimensions this implies the existence of two commuting Abelian isometries, time translation and axial rotation, which are extremely powerful; by integrating out the two isometries from the theory, we obtain an integrable two-dimensional $G L(2, \mathbb{R})$ sigma-model. The literature on these theories is enormous and many solution-generating techniques are available, which provide a variety of derivations of the Kerr solution.

There are two natural ways of extending axial symmetry to higher dimensions. We may look for solutions invariant under the group $O(d-2)$ of spatial rotations around a given line axis, where the orbits of $O(d-2)$ are $(d-3)$-spheres. However, in more than four dimensions these orbits have nonzero curvature. As a consequence, after dimensional reduction of these orbits, the sigma model acquires terms (of exponential type) that prevent a straightforward integration of the equations (see $[34,35]$ for an investigation of these equations).

This suggests that one should look for a different higher-dimensional extension of the fourdimensional axial symmetry. Instead of rotations around a line, consider rotations around (spatial) codimension- 2 hypersurfaces. These are $U(1)$ symmetries. If we assume $d-3$ commuting $U(1)$ symmetries, so that we have a spatial $U(1)^{d-3}$ symmetry in addition to the timelike symmetry $\mathbb{R}$, then the vacuum Einstein equations again reduce to an integrable two-dimensional $G L(d-2, \mathbb{R})$ sigma model with powerful solution-generating techniques.

However, there is an important limitation: only in $d=4,5$ can these geometries be globally asymptotically flat. Global asymptotic flatness implies an asymptotic factor $S^{d-2}$ in the spatial geometry, whose isometry group $O(d-1)$ has a Cartan subgroup $U(1)^{N}$. If, as above, we demand $d-3$ axial isometries, then, asymptotically, these symmetries must approach elements of $O(d-1)$, so we need $U(1)^{d-3} \subset U(1)^{N}$, i.e.,

$$
d-3 \leq N=\left\lfloor\frac{d-1}{2}\right\rfloor,
$$

which is only possible in $d=4,5$. This is the main reason for the recent great progress in the construction of exact five-dimensional black holes, and the failure to extend it to $d>5$.

Finally, the classification of possible horizon topologies becomes increasingly complicated in higher dimensions [98]. In four spacetime dimensions the (spatial section of the) horizon is a twodimensional surface, so the possible topologies can be easily characterized and restricted. Much less restriction is possible as $d$ is increased.

All these aspects will be discussed in more detail below. 


\section{Scope and Organization of this Article}

\section{$2.1 \quad$ Scope}

The emphasis of this article is on classical properties of exact higher-dimensional black-hole solutions. We devote most space to a rather pedagogical discussion of vacuum solutions. Since this includes black rings, there is some overlap with our earlier review [84]. The present review discusses material that has appeared since [84], in particular the "doubly spinning" black-ring solution of [212]. However, we shall not discuss several aspects of black-ring physics that were dealt with at length in [84], for example, black-ring microphysics. On the other hand, we present some new material: Figures 3, 6, 7, 13, and 14 describe the physical parameter ranges (phase space) of higher-dimensional black holes, and Figure 4 for the area of 5D Myers-Perry solutions, have not been presented earlier. Some of our discussion of the properties of the solutions is also new.

Our discussion of nonvacuum black holes is less pedagogical than that of the vacuum solutions. It is essentially a survey of the literature. In going beyond vacuum solutions, we had to decide what kinds of matter fields to consider. Since much of the motivation for the study of extra dimensions comes from string theory, we have restricted ourselves to considering black-hole solutions of supergravity theories known to arise as consistent truncations of $d=10,11$ supergravity. We consider both asymptotically flat and asymptotically anti-de Sitter black holes.

In the asymptotically flat case, we consider only solutions of maximal supergravity theories arising from the toroidal reduction of $d=10,11$ supergravity to five or more dimensions. In particular, this implies that in five dimensions we demand the presence of a Chern-Simons term for the gauge field, with a precise coefficient. A review of charged rotating black holes with other values for the Chern-Simons coupling can be found in [161].

In the asymptotically AdS case, we consider solutions of gauged supergravity theories arising from the dimensional reduction of $d=10,11$ supergravity on spheres, in particular the maximal-gauged supergravity theories in $d=4,5,7$. Obviously $d=4$ does not fall within our "higher-dimensional" remit but asymptotically $\mathrm{AdS} d=4$ black holes are not as familiar as their asymptotically-flat cousins so it seems worthwhile reviewing them here. In the AdS case, several different asymptotic boundary conditions are of physical interest. We consider only black holes obeying standard "normalizable" boundary conditions [1]. Note that all known black-hole solutions satisfying these restrictions involve only Abelian gauge fields.

Important related subjects that we do not discuss include: black holes in brane-world scenarios (reviewed in [186]); black holes in spacetimes with Kaluza-Klein asymptotics (reviewed in [129]), and in general black holes with different asymptotics than flat or AdS; black holes in higherderivative theories [199, 33]; black-hole formation at the LHC or in cosmic rays, and the spectrum of their radiation (reviewed in [30, 155]).

\subsection{Organization}

Sections 3 to 6 are devoted to asymptotically-flat vacuum solutions: Section 3 introduces basic notions and solutions, in particular the Schwarzschild-Tangherlini black hole. Section 4 presents the Myers-Perry solutions, first with a single angular momentum, then with arbitrary rotation. Section 5 reviews the great recent progress in five-dimensional vacuum black holes: first we discuss black rings, with one and two angular momenta; then we introduce the general analysis of solutions with two rotational isometries (or $d-3$, in general). In Section 6 we briefly describe a first attempt at understanding $d \geq 6$ vacuum black holes beyond the MP solutions.

Section 7 reviews asymptotically-flat black holes with gauge fields (within the restricted class mentioned above). Section 8 concludes our overview of asymptotically-flat solutions (vacuum and charged) with a discussion of general results and some open problems. Finally, Section 9 reviews asymptotically AdS black-hole solutions of gauged supergravity theories. 


\section{Basic Concepts and Solutions}

In this section we present the basic framework for general relativity in higher dimensions, beginning with the definition of conserved charges in vacuum, i.e., mass and angular momentum, and the introduction of a set of dimensionless variables that are convenient for describing the phase space and phase diagrams of higher-dimensional rotating black holes. Then we introduce the Tangherlini solutions that generalize the four-dimensional Schwarzschild solution. The analysis that proves their classical stability is then reviewed. Black strings and black $p$-branes, and their GregoryLaflamme instability, are briefly discussed for their relevance to novel kinds of rotating black holes.

\subsection{Conserved charges}

The Einstein-Hilbert action is generalized to higher dimensions in the form

$$
I=\frac{1}{16 \pi G} \int d^{d} x \sqrt{-g} R+I_{\text {matter }} .
$$

This is a straightforward generalization, and the only aspect that deserves some attention is the implicit definition of Newton's constant $G$ in $d$ dimensions. It enters the Einstein equations in the conventional form

$$
R_{\mu \nu}-\frac{1}{2} g_{\mu \nu} R=8 \pi G T_{\mu \nu}
$$

where $T_{\mu \nu}=2(-g)^{-1 / 2}\left(\delta I_{\text {matter }} / \delta g^{\mu \nu}\right)$. This definition of the gravitational coupling constant, without any additional dimension-dependent factors, has the notable advantage that the BekensteinHawking entropy formula takes the same form

$$
S=\frac{\mathcal{A}_{H}}{4 G}
$$

in every dimension. This follows, e.g., from the standard Euclidean quantum gravity calculation of the entropy.

Mass, angular momenta, and other conserved charges of isolated systems are defined through comparison to the field created near asymptotic infinity by a weakly gravitating system ([154] gives a careful Hamiltonian analysis of conserved charges in higher-dimensional asymptotically-flat spacetimes). The Einstein equations for a small perturbation around flat Minkowski space

$$
g_{\mu \nu}=\eta_{\mu \nu}+h_{\mu \nu}
$$

in linearized approximation take the conventional form

$$
\square \bar{h}_{\mu \nu}=-16 \pi G T_{\mu \nu}
$$

where $\bar{h}_{\mu \nu}=h_{\mu \nu}-\frac{1}{2} h \eta_{\mu \nu}$ and we have imposed the transverse gauge condition $\nabla_{\mu} \bar{h}^{\mu \nu}=0$.

Since the sources are localized and we work at linearized perturbation order, the fields in the asymptotic region are the same as those created by point-like sources of mass $M$ and angular momentum with antisymmetric matrix $J_{i j}$, at the origin $x^{k}=0$ of flat space in Cartesian coordinates,

$$
\begin{aligned}
& T_{t t}=M \delta^{(d-1)}\left(x^{k}\right), \\
& T_{t i}=-\frac{1}{2} J_{i j} \nabla_{j} \delta^{(d-1)}\left(x^{k}\right) .
\end{aligned}
$$

The equations are easily integrated, assuming stationarity, to find

$$
\begin{aligned}
\bar{h}_{t t} & =\frac{16 \pi G}{(d-3) \Omega_{d-2}} \frac{M}{r^{d-3}}, \\
\bar{h}_{t i} & =-\frac{8 \pi G}{\Omega_{d-2}} \frac{x^{k} J_{k i}}{r^{d-1}},
\end{aligned}
$$


where $r=\sqrt{x^{i} x^{i}}$, and $\Omega_{d-2}=2 \pi^{(d-1) / 2} / \Gamma\left(\frac{d-1}{2}\right)$ is the area of a unit $(d-2)$-sphere. From here we recover the metric perturbation $h_{\mu \nu}=\bar{h}_{\mu \nu}-\frac{1}{d-2} \bar{h} \eta_{\mu \nu}$ as

$$
\begin{aligned}
h_{t t} & =\frac{16 \pi G}{(d-2) \Omega_{d-2}} \frac{M}{r^{d-3}}, \\
h_{i j} & =\frac{16 \pi G}{(d-2)(d-3) \Omega_{d-2}} \frac{M}{r^{d-3}} \delta_{i j}, \\
h_{t i} & =-\frac{8 \pi G}{\Omega_{d-2}} \frac{x^{k} J_{k i}}{r^{d-1}} .
\end{aligned}
$$

It is often convenient to have the off-diagonal rotation components of the metric in a different form. By making a suitable coordinate rotation the angular momentum matrix $J_{i j}$ can be put into block-diagonal form, each block being a $2 \times 2$ antisymmetric matrix with parameter

$$
J_{a} \equiv J_{2 a-1,2 a} .
$$

Here $a=1, \ldots, N$ labels the different independent rotation planes. If we introduce polar coordinates on each of the planes

$$
\left(x_{2 a-1}, x_{2 a}\right)=\left(r_{a} \cos \phi_{a}, r_{a} \sin \phi_{a}\right)
$$

then (no sum over $a)$

$$
h_{t \phi_{a}}=-\frac{8 \pi G J_{a}}{\Omega_{d-2}} \frac{r_{a}^{2}}{r^{d-1}}=-\frac{8 \pi G J_{a}}{\Omega_{d-2}} \frac{\mu_{a}^{2}}{r^{d-3}} .
$$

In the last expression we have introduced the 'direction cosines'

$$
\mu_{a}=\frac{r_{a}}{r}
$$

Given the abundance of black-hole solutions in higher dimensions, one is interested in comparing properties, such as the horizon area $\mathcal{A}_{H}$, of different solutions characterized by the same set of parameters $\left(M, J_{a}\right)$. A meaningful comparison between dimensionful magnitudes requires the introduction of a common scale, so the comparison is made between dimensionless magnitudes obtained by factoring out this scale. Since classical general relativity in vacuum is scale invariant, the common scale must be one of the physical parameters of the solutions, and a natural choice is the mass. Thus we introduce dimensionless quantities for the spins $j_{a}$ and the area $a_{H}$,

$$
j_{a}^{d-3}=c_{J} \frac{J_{a}^{d-3}}{G M^{d-2}}, \quad a_{H}^{d-3}=c_{\mathcal{A}} \frac{\mathcal{A}_{H}^{d-3}}{(G M)^{d-2}},
$$

where the numerical constants are

$$
c_{J}=\frac{\Omega_{d-3}}{2^{d+1}} \frac{(d-2)^{d-2}}{(d-3)^{\frac{d-3}{2}}}, \quad c_{\mathcal{A}}=\frac{\Omega_{d-3}}{2(16 \pi)^{d-3}}(d-2)^{d-2}\left(\frac{d-4}{d-3}\right)^{\frac{d-3}{2}}
$$

(these definitions follow the choices in [80]). Studying the entropy, or the area $\mathcal{A}_{H}$, as a function of $J_{a}$ for fixed mass is equivalent to finding the function $a_{H}\left(j_{a}\right)$.

Note that, with our definition of the gravitational constant $G$, both the Newtonian gravitational potential energy,

$$
\Phi=-\frac{1}{2} h_{t t}
$$

and the force law (per unit mass)

$$
\mathbf{F}=-\nabla \Phi=\frac{(d-3) 8 \pi G}{(d-2) \Omega_{d-2}} \frac{M}{r^{d-2}} \hat{\mathbf{r}}
$$


acquire $d$-dependent numerical prefactors. Had we chosen to define Newton's constant so as to absorb these factors in the expressions for $\Phi$ or $\mathbf{F}$, Equation (7) would have been more complicated.

To warm up before dealing with black holes, we follow John Michell and Simon de Laplace and compute, using Newtonian mechanics, the radius at which the escape velocity of a test particle in this field reaches the speed of light. The kinetic energy of a particle of unit mass with velocity $v=c=1$ is $K=1 / 2$, so the equation $K+\Phi=0$ that determines the Michell-Laplace 'horizon' radius is

$$
h_{t t}\left(r=r_{M L}\right)=1 \Rightarrow r_{M L}=\left(\frac{16 \pi G M}{(d-2) \Omega_{d-2}}\right)^{\frac{1}{d-3}} .
$$

We will see in the next section 3.2 that, just like in four dimensions, this is precisely equal to the horizon radius for a static black hole in higher dimensions.

\subsection{The Schwarzschild-Tangherlini solution and black $p$-branes}

Consider the linearized solution above for a static source (14) in spherical coordinates, and pass to a gauge where $r$ is the area radius,

$$
r \rightarrow r-\frac{8 \pi G}{(d-2)(d-3) \Omega_{d-2}} \frac{M}{r^{d-3}} .
$$

The linearized approximation to the field of a static source is then

$$
d s_{(\text {lin })}^{2}=-\left(1-\frac{\mu}{r^{d-3}}\right) d t^{2}+\left(1+\frac{\mu}{r^{d-3}}\right) d r^{2}+r^{2} d \Omega_{d-2}^{2},
$$

where, to lighten the notation, we have introduced the 'mass parameter'

$$
\mu=\frac{16 \pi G M}{(d-2) \Omega_{d-2}} .
$$

This suggests that the Schwarzschild solution generalizes to higher dimensions in the form

$$
d s^{2}=-\left(1-\frac{\mu}{r^{d-3}}\right) d t^{2}+\frac{d r^{2}}{1-\frac{\mu}{r^{d-3}}}+r^{2} d \Omega_{d-2}^{2} .
$$

In essence, all we have done is change the radial falloff $1 / r$ of the Newtonian potential to the $d$-dimensional one, $1 / r^{d-3}$. As Tangherlini found in 1963 [232], this turns out to give the correct solution: it is straightforward to check that this metric is indeed Ricci flat. It is apparent that there is an event horizon at $r_{0}=\mu^{1 /(d-3)}$, which coincides with the Michell-Laplace result (25).

Having this elementary class of black-hole solutions, it is easy to construct other vacuum solutions with event horizons in $d \geq 5$. The direct product of two Ricci-flat manifolds is itself a Ricci-flat manifold. So, given any vacuum black-hole solution $\mathcal{B}$ of the Einstein equations in $d$ dimensions, the metric

$$
d s_{d+p}^{2}=d s_{d}^{2}(\mathcal{B})+\sum_{i=1}^{p} d x^{i} d x^{i}
$$

describes a black $p$-brane, in which the black-hole horizon $\mathcal{H} \subset \mathcal{B}$ is extended to a horizon $\mathcal{H} \times \mathbb{R}^{p}$, or $\mathcal{H} \times \mathbb{T}^{p}$ if we identify periodically $x^{i} \sim x^{i}+L_{i}$. A simple way of obtaining another kind of vacuum solution is the following: unwrap one of the directions $x^{i}$, perform a boost $t \rightarrow \cosh \alpha t+$ $\sinh \alpha x^{i}, x^{i} \rightarrow \sinh \alpha t+\cosh \alpha x^{i}$, and re-identify points periodically along the new coordinate $x^{i}$. Although locally equivalent to the static black brane, the new boosted black-brane solution is globally different from it.

These black brane spacetimes are not (globally) asymptotically flat, so we only introduce them insofar as they are relevant for understanding the physics of asymptotically-flat black holes. 


\subsection{Stability of the static black hole}

The stability of the $d>4$ Schwarzschild solution against linearized gravitational perturbations can be analyzed by decomposing such perturbations into scalar, vector and tensor types according to how they transform under the rotational-symmetry group $S O(d-1)$ [105, 163, 151]. Assuming a time dependence $e^{-i \omega t}$ and expanding in spherical harmonics on $S^{d-2}$, the equations governing each type of perturbation reduce to a single ODE governing the radial dependence. This equation can be written in the form of a time-independent Schrödinger equation with "energy" eigenvalue $\omega^{2}$.

In investigating stability, we consider perturbations that are regular on the future horizon and outgoing at infinity. An instability would correspond to a mode with $\operatorname{Im} \omega>0$. For such modes, the boundary conditions at the horizon and infinity imply that the left-hand side (LHS) of the Schrödinger equation is self-adjoint, and hence $\omega^{2}$ is real. Therefore, an unstable mode must have negative imaginary $\omega$. For tensor modes, the potential in the Schrödinger equation is manifestly positive, hence $\omega^{2}>0$ and there is no instability [105]. For vectors and scalars, the potential is not everywhere positive. Nevertheless, it can be shown that the operator appearing on the LHS of the Schrödinger equation is positive, hence $\omega^{2}>0$ and there is no instability [151]. In conclusion, the $d>4$ Schwarzschild solution is stable against linearized gravitational perturbations.

\subsection{Gregory-Laflamme instability}

The instabilities of black strings and black branes [118, 119] have been reviewed in [167, 128], so we shall be brief in this section and only mention the features that are most relevant to our subject. We shall only discuss neutral black holes and black branes; when charges are present, the problem becomes more complex.

This instability is the prototype for situations in which the size of the horizon is much larger in some directions than in others. Consider, as a simple, extreme case of this, the black string obtained by adding a flat direction $z$ to the Schwarzschild solution. One can decompose linearized gravitational perturbations into scalar, vector and tensor types according to how they transform with respect to transformations of the Schwarzschild coordinates. Scalar and vector perturbations of this solution are stable [117]. Tensor perturbations that are homogeneous along the $z$-direction are also stable, since they are the same as tensor perturbations of the Schwarzschild black hole. However, there appears to be an instability for long-wavelength tensor perturbations with nontrivial dependence on $z$; the frequency $\omega$ of perturbations $\sim e^{-i(\omega t-k z)}$ acquires a positive imaginary part when $k<k_{G L} \sim 1 / r_{0}$, where $r_{0}$ is the Schwarzschild horizon radius. Thus, if the string is compactified on a circle of length $L>2 \pi / k_{G L} \sim r_{0}$, it becomes unstable. Of the unstable modes, the fastest one (with the largest imaginary frequency) occurs for $k$ roughly one half of $k_{G L}$. The instability creates inhomogeneities along the direction of the string. Their evolution beyond the linear approximation has been followed numerically in [42]. It is unclear yet what the endpoint is; the inhomogeneities may well grow until a sphere pinches down to a singularity. ${ }^{3}$ In this case, the Planck scale will be reached along the evolution, and fragmentation of the black string into black holes, with a consistent increase in the total horizon entropy, may occur.

Another important feature of this phenomenon is the appearance of a zero-mode (i.e., static) perturbation with $k=k_{G L}$. Perturbing the black string with this mode yields a new static solution with inhomogeneities along the string direction [117, 120]. Following numerically these static perturbations beyond the linear approximation has given a new class of inhomogeneous black strings [248].

These results easily generalize to black $p$-branes; for a wavevector $\mathbf{k}$ along the $p$ directions

\footnotetext{
${ }^{3}$ It has been shown that this requires an infinite affine parameter distance along the null-geodesics generators of the horizon [142]. However, it may still take finite time as measured by an external observer [190].
} 
tangent to the brane, the perturbations $\sim \exp (-i \omega t+i \mathbf{k} \cdot \mathbf{z}))$ with $|\mathbf{k}| \leq k_{G L}$ are unstable. The value of $k_{G L}$ depends on the codimension of the black brane, but not on $p$. 


\section{Myers-Perry Solutions}

The generalization of the Schwarzschild solution to $d>4$ is, as we have seen, a rather straightforward problem. However, in general relativity it is often very difficult to extend a solution from the static case to the stationary one (as exemplified by the Kerr solution). Impressively, in 1986 Myers and Perry (MP) managed to find exact solutions for black holes in any dimension $d>4$, rotating in all possible independent rotation planes [200]. This feat was possible as the solutions belong in the Kerr-Schild class

$$
g_{\mu \nu}=\eta_{\mu \nu}+2 H\left(x^{\rho}\right) k_{\mu} k_{\nu},
$$

where $k_{\mu}$ is a null vector with respect to both $g_{\mu \nu}$ and the Minkowski metric $\eta_{\mu \nu}$. This entails a sort of linearization of the problem, which facilitates greatly the resolution of the equations. Of all known vacuum black holes in $d>4$, only the Myers-Perry solutions seem to have this property.

In this section, we review these solutions and their properties, beginning from black holes with a single rotation, and then extending them to arbitrary rotation. The existence of ultraspinning regimes in $d \geq 6$ is emphasized. The symmetries and stability of the MP solutions are also discussed.

\subsection{Rotation in a single plane}

Let us begin with solutions that rotate in a single plane. These are not only simpler, but also exhibit more clearly the qualitatively new physics afforded by the additional dimensions.

The metric takes the form

$$
\begin{aligned}
d s^{2}= & -d t^{2}+\frac{\mu}{r^{d-5} \Sigma}\left(d t-a \sin ^{2} \theta d \phi\right)^{2}+\frac{\Sigma}{\Delta} d r^{2}+\Sigma d \theta^{2}+\left(r^{2}+a^{2}\right) \sin ^{2} \theta d \phi^{2} \\
& +r^{2} \cos ^{2} \theta d \Omega_{(d-4)}^{2}
\end{aligned}
$$

where

$$
\Sigma=r^{2}+a^{2} \cos ^{2} \theta, \quad \Delta=r^{2}+a^{2}-\frac{\mu}{r^{d-5}} .
$$

The physical mass and angular momentum are easily obtained by comparing the asymptotic field to Equations (14) and (19), and are given in terms of the parameters $\mu$ and $a$ by

$$
M=\frac{(d-2) \Omega_{d-2}}{16 \pi G} \mu, \quad J=\frac{2}{d-2} M a .
$$

Hence, one can think of $a$ as essentially the angular momentum per unit mass. We can choose $a \geq 0$ without loss of generality. ${ }^{4}$

As in Tangherlini's solution, this metric seems to follow from a rather straightforward extension of the Kerr solution, which is recovered when $d=4$. The first line in Equation (32) looks indeed like the Kerr solution, with the $1 / r$ falloff replaced, in appropriate places, by $1 / r^{d-3}$. The second line contains the line element on a $(d-4)$-sphere, which accounts for the additional spatial dimensions. It might, therefore, seem that, again, the properties of these black holes should not differ much from their four-dimensional counterparts.

However, this is not the case. Heuristically, we can see the competition between gravitational attraction and centrifugal repulsion in the expression

$$
\frac{\Delta}{r^{2}}-1=-\frac{\mu}{r^{d-3}}+\frac{a^{2}}{r^{2}}
$$

${ }^{4}$ This choice corresponds to rotation in the positive sense (i.e., increasing $\phi$ ). The solution presented in [200] is obtained by $\phi \rightarrow-\phi$, which gives rotation in a negative sense. 
Roughly, the first term on the right-hand side (RHS) corresponds to the attractive gravitational potential and falls off in a dimension-dependent fashion. In contrast, the repulsive centrifugal barrier described by the second term does not depend on the total number of dimensions, since rotations always refer to motions in a plane.

Given the similarities between Equation (32) and the Kerr solution, it is clear that the outer event horizon lies at the largest (real) root $r_{0}$ of $g_{r r}^{-1}=0$, i.e., $\Delta(r)=0$. Thus, we expect that the features of the event horizons will be strongly dimension dependent, and this is indeed the case. If there is an event horizon at $r=r_{0}$,

$$
r_{0}^{2}+a^{2}-\frac{\mu}{r_{0}^{d-5}}=0
$$

its area will be

$$
\mathcal{A}_{H}=r_{0}^{d-4}\left(r_{0}^{2}+a^{2}\right) \Omega_{d-2} .
$$

For $d=4$, a regular horizon is present for values of the spin parameter $a$ up to the Kerr bound: $a=\mu / 2$ (or $a=G M)$, which corresponds to an extremal black hole with a single degenerate horizon (with vanishing surface gravity). Solutions with $a>G M$ correspond to naked singularities. In $d=5$, the situation is apparently quite similar since the real root at $r_{0}=\sqrt{\mu-a^{2}}$ exists only up to the extremal limit $\mu=a^{2}$. However, this extremal solution has zero area, and in fact, has a naked ring singularity.

For $d \geq 6, \Delta(r)$ is always positive at large values of $r$, but the term $-\mu / r^{d-5}$ makes it negative at small $r$ (we are assuming positive mass). Therefore $\Delta$ always has a (single) positive real root independent of the value of $a$. Hence, regular black-hole solutions exist with arbitrarily large $a$. Solutions with large angular momentum per unit mass are referred to as "ultraspinning".

An analysis of the shape of the horizon in the ultraspinning regime $a \gg r_{0}$ shows that the black holes flatten along the plane of rotation [81]; the extent of the horizon along this plane is $\sim a$, while, in directions transverse to this plane, its size is $\sim r_{0}$. In fact, a limit can be taken in which the ultraspinning black hole becomes a black membrane with horizon geometry $\mathbb{R}^{2} \times S^{d-4}$. This turns out to have important consequences for black holes in $d \geq 6$, as we will discuss later. The transition between the regime in which the black hole behaves like a fairly compact, Kerr-like object, and the regime in which it is better characterized as a membrane, is most clearly seen by analyzing the black hole temperature

$$
T_{H}=\frac{1}{4 \pi}\left(\frac{2 r_{0}}{r_{0}^{2}+a^{2}}+\frac{d-5}{r_{0}}\right) .
$$

At

$$
\left(\frac{a}{r_{0}}\right)_{\text {mem }}=\sqrt{\frac{d-3}{d-5}}
$$

this temperature reaches a minimum. For $a / r_{0}$ smaller than this value, quantities like $T_{H}$ and $\mathcal{A}_{H}$ decrease, in a manner similar to the Kerr solution. However, past this point they rapidly approach the black membrane results in which $T_{H} \sim 1 / r_{0}$ and $\mathcal{A}_{H} \sim a^{2} r_{0}^{d-4}$, with $a^{2}$ characterizing the area of the membrane worldvolume.

The properties of the solutions are conveniently encoded using the dimensionless variables $a_{H}$, $j$ introduced in Equation (21). For the solutions (32) the curve $a_{H}(j)$ can be found in parametric form, in terms of the dimensionless 'shape' parameter $\nu=\frac{r_{0}}{a}$, as

$$
\begin{aligned}
j^{d-3} & =\frac{\pi}{(d-3)^{\frac{d-3}{2}}} \frac{\Omega_{d-3}}{\Omega_{d-2}} \frac{\nu^{5-d}}{1+\nu^{2}}, \\
a_{H}^{d-3} & =8 \pi\left(\frac{d-4}{d-3}\right)^{\frac{d-3}{2}} \frac{\Omega_{d-3}}{\Omega_{d-2}} \frac{\nu^{2}}{1+\nu^{2}} .
\end{aligned}
$$

Living Reviews in Relativity

http://www . livingreviews . org/lrr-2008-6 
The static and ultraspinning limits correspond to $\nu \rightarrow \infty$ and $\nu \rightarrow 0$, respectively. These curves are represented for $d=5,6$, and 10 in Figure 1 . The inflection point where $d^{2} a_{H} / d j^{2}$ changes sign when $d \geq 6$, occurs at the value (39).

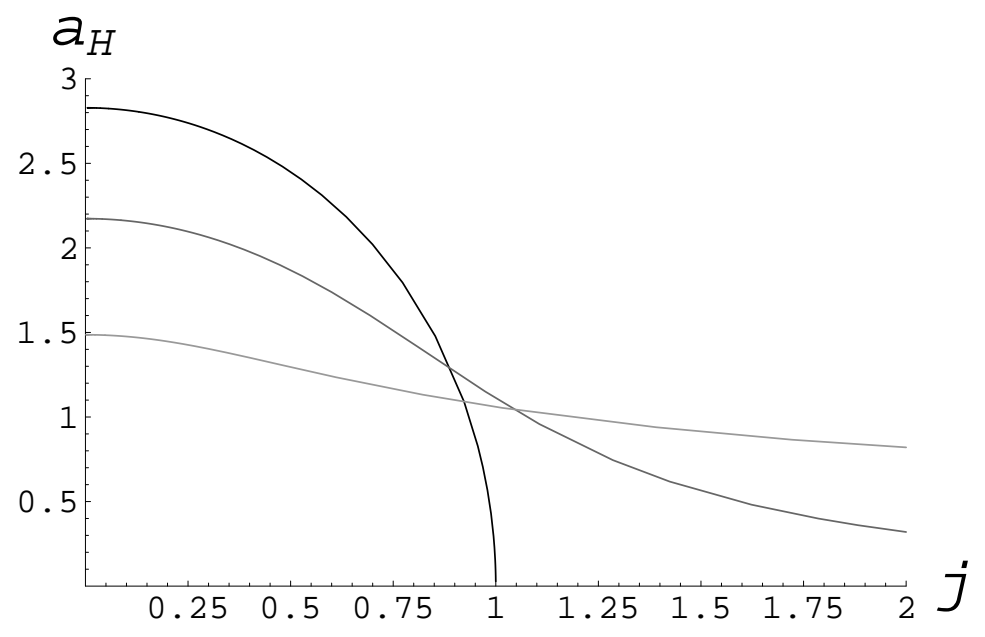

Figure 1: Horizon area vs. angular momentum for Myers-Perry black holes with a single spin in $d=5$ (black), $d=6$ (dark gray), and $d=10$ (light gray).

\subsection{General solution}

[200] also gives black-hole solutions with arbitrary rotation in each of the $N \equiv\left\lfloor\frac{d-1}{2}\right\rfloor$ independent rotation planes. The cases of odd and even $d$ are slightly different. When $d$ is odd, the solution is

$$
d s^{2}=-d t^{2}+\left(r^{2}+a_{i}^{2}\right)\left(d \mu_{i}^{2}+\mu_{i}^{2} d \phi_{i}^{2}\right)+\frac{\mu r^{2}}{\Pi F}\left(d t-a_{i} \mu_{i}^{2} d \phi_{i}\right)^{2}+\frac{\Pi F}{\Pi-\mu r^{2}} d r^{2} .
$$

Here and below $i=1, \ldots, N$ and we assume summation over $i$. The mass parameter is $\mu$, not to be confused with the direction cosines $\mu_{i}$, which satisfy $\mu_{i}^{2}=1$. For even $d$, the general solution is

$$
d s^{2}=-d t^{2}+r^{2} d \alpha^{2}+\left(r^{2}+a_{i}^{2}\right)\left(d \mu_{i}^{2}+\mu_{i}^{2} d \phi_{i}^{2}\right)+\frac{\mu r}{\Pi F}\left(d t-a_{i} \mu_{i}^{2} d \phi_{i}\right)^{2}+\frac{\Pi F}{\Pi-\mu r} d r^{2},
$$

where now $\mu_{i}^{2}+\alpha^{2}=1$.

For both cases we can write the functions $F\left(r, \mu_{i}\right)$ and $\Pi(r)$ as

$$
F\left(r, \mu_{i}\right)=1-\frac{a_{i}^{2} \mu_{i}^{2}}{r^{2}+a_{i}^{2}}, \quad \Pi(r)=\prod_{i=1}^{N}\left(r^{2}+a_{i}^{2}\right) .
$$

The relation between $\mu$ and $a_{i}$ and the mass and angular momenta is the same as in Equation (34). The event horizon is again at the largest real root of $g^{r r}$, that is,

$$
\Pi\left(r_{0}\right)-\mu r_{0}^{2}=0 \quad(\text { odd } d), \quad \Pi\left(r_{0}\right)-\mu r_{0}=0 \quad(\text { even } d) .
$$

The horizon area is

$$
\mathcal{A}_{H}=\frac{\Omega_{d-2}}{2 \kappa} \mu\left(d-3-\frac{2 a_{i}^{2}}{r_{0}^{2}+a_{i}^{2}}\right)
$$


and the surface gravity $\kappa$ is

$$
\kappa=\lim _{r \rightarrow r_{0}} \frac{\Pi^{\prime}-2 \mu r}{2 \mu r^{2}} \quad(\text { odd } d), \quad \kappa=\lim _{r \rightarrow r_{0}} \frac{\Pi^{\prime}-\mu}{2 \mu r} \quad(\text { even } d) .
$$

Extremal solutions are obtained when $\kappa=0$ at the event horizon.

\subsubsection{Phase space}

The determination of $r_{0}$ involves an equation of degree $2 N$, which in general is difficult, if not impossible, to solve algebraically. So the presence of horizons for generic parameters in Equation (42) and (43) is difficult to ascertain. Nevertheless, a number of features, in particular the ultraspinning regimes that are important in the determination of the allowed parameter range, can be analyzed.

Following Equation (21), we can fix the mass and define dimensionless quantities $j_{i}$ for each of the angular momenta. Up to a normalization constant, the rotation parameters $a_{i}$ at fixed mass are equivalent to the $j_{i}$. We take $\left(j_{1}, \ldots, j_{N}\right)$ as the coordinates in the phase space of solutions. We aim to determine the region in this space that corresponds to actual black-hole solutions.

Consider first the case in which all spin parameters are nonzero. Then an upper extremality bound on a combination of the spins arises. If it is exceeded, naked singularities appear, as in the $d=4$ Kerr black hole [200]. So we can expect that, as long as all spin parameters take values not too dissimilar, $j_{1} \sim j_{2} \sim \cdots \sim j_{N}$, all spins must remain parametrically $O(1)$, i.e., there is no ultraspinning regime in which all $j_{i} \gg 1$.

Next, observe that for odd $d$, a sufficient (but not necessary) condition for the existence of a horizon is that any two of the spin parameters vanish, i.e., if two $a_{i}$ vanish, a horizon will always exist, irrespective of how large the remaining spin parameters are. For even $d$, the existence of a horizon is guaranteed if any one of the spins vanishes. Thus, arbitrarily large (i.e., ultraspinning) values can be achieved for all but two (one) of the $j_{i}$ in odd (even) dimensions.

Assume, then, an ultraspinning regime in which $n$ rotation parameters are comparable among themselves, and much larger than the remaining $N-n$ ones. A limit then exists to a black $2 n$ brane of limiting horizon topology $S^{d-2} \rightarrow \mathbb{R}^{2 n} \times S^{d-2(n+1)}$. The limiting geometry is in fact the direct product of $\mathbb{R}^{2 n}$ and a $(d-2 n)$-dimensional Myers-Perry black hole [81]. Thus, in an ultraspinning regime the allowed phase space of $d$-dimensional black holes can be inferred from that of $(d-2 n)$-dimensional black holes. Let us then begin from $d=5,6$ and proceed to higher $d$.

The phase space is fairly easy to determine in $d=5,6$; see Figure 2 . In $d=5$ Equation (45) admits a real root for

$$
\left|j_{1}\right|+\left|j_{2}\right| \leq 1
$$

which is a square, with extremal solutions at the boundaries, where the inequality is saturated. These extremal solutions have regular horizons if, and only if, both angular momenta are nonvanishing. There are no ultraspinning regimes: our arguments above relate this fact to the nonexistence of three-dimensional vacuum black holes.

In $d=6$ the phase space of regular black-hole solutions is again bounded by a curve of extremal black holes. In terms of the dimensionless parameter $\nu=r_{0} / \mu^{1 / 3}$, the extremal curve is

$$
\left|j_{1}\right|=\left(\frac{\pi}{2 \sqrt{3}}\right)^{1 / 3} \sqrt{\frac{1-4 \nu^{3} \pm \sqrt{1-16 \nu^{3}}}{4 \nu}}, \quad\left|j_{2}\right|=\left(\frac{\pi}{2 \sqrt{3}}\right)^{1 / 3} \sqrt{\frac{1-4 \nu^{3} \mp \sqrt{1-16 \nu^{3}}}{4 \nu}},
$$

with $0 \leq \nu \leq 2^{-4 / 3}$. As $\nu \rightarrow 0$ we get into the ultraspinning regimes, in which one of the spins diverges while the other vanishes, according to the general behavior discussed above. In this regime, at, say, constant large $j_{1}$, the solutions approach a Kerr black membrane and thus the available phase space is of the form $j_{2} \leq f\left(j_{1}\right)$, i.e., a rescaled version of the Kerr bound $j \leq 1 / 4$. Functions such as $a_{H}$ can be recovered from the four-dimensional solutions. 
(a)

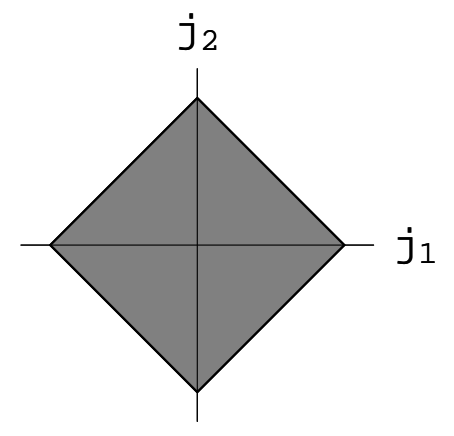

(b)

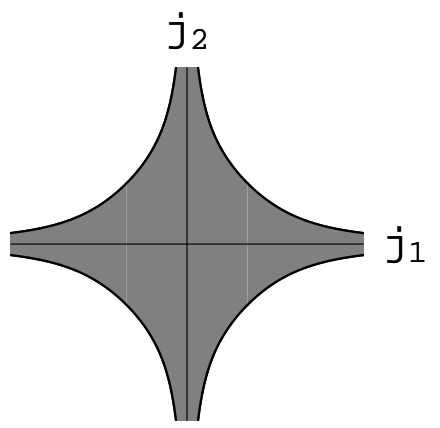

Figure 2: Phase space of (a) five-dimensional and (b) six-dimensional MP rotating black holes: black holes exist for parameters within the shaded regions. The boundaries of the phase space correspond to extremal black holes with regular horizons, except at the corners of the square in five dimensions, where they become naked singularities. The six-dimensional phase space extends along the axes to arbitrarily large values of each of the two angular momenta (ultraspinning regimes).

In $d=7$, with three angular momenta $j_{1}, j_{2}, j_{3}$, it is more complicated to obtain the explicit form of the surface of extremal solutions that bind the phase space of MP black holes, but it is still possible to sketch it; see Figure 3(a). There are ultraspinning regimes in which one of the angular momenta becomes much larger than the other two. In this limit the phase space of solutions at, say, large $j_{3}$, becomes asymptotically of the form $\left|j_{1}\right|+\left|j_{2}\right| \leq f\left(j_{3}\right)$, i.e., of the same form as the five-dimensional phase space (48), only rescaled by a factor $f\left(j_{3}\right)$ (which vanishes as $j_{3} \rightarrow \infty$ ).

A similar 'reduction' to a phase space in two fewer dimensions along ultraspinning directions appears in the phase space of $d=8 \mathrm{MP}$ black holes; see Figure 3(b); a section at constant large $j_{3}$ becomes asymptotically of the same shape as the six-dimensional diagram (49), rescaled by a $j_{3}$-dependent factor.

These examples illustrate how we can infer the qualitative form of the phase space in dimension $d$ if we know it in $d-2$, e.g., in $d=9,10$, with four angular momenta, the sections of the phase space at large $j_{4}$ approach the shapes in Figure 3 (a) and (b), respectively.

If we manage to determine the regime of parameters where regular black holes exist, we can express other (dimensionless) physical magnitudes as functions of the phase-space variables $j_{a}$. Figure 4 is a plot of the area function $a_{H}\left(j_{1}, j_{2}\right)$ in $d=5$, showing only the quadrant $j_{1}, j_{2} \geq 0$; the complete surface allowing $j_{1}, j_{2}<0$ is a tent-like dome. In $d=6$ the shape of the area surface is a little more complicated to draw, but it can be visualized by combining the information from the plots we have presented in this section. In general, the 'ultraspinning reduction' to $d-2 n$ dimensions also yields information about the area and other properties of the black holes. 


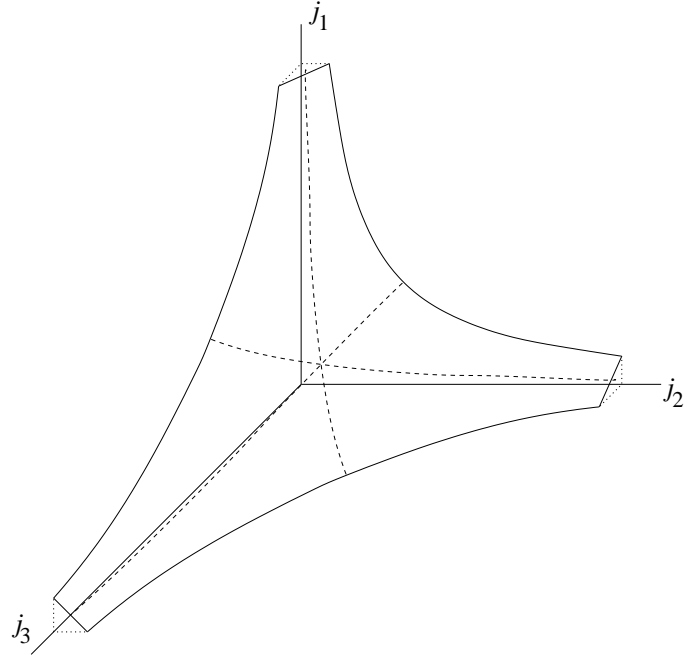

(a)

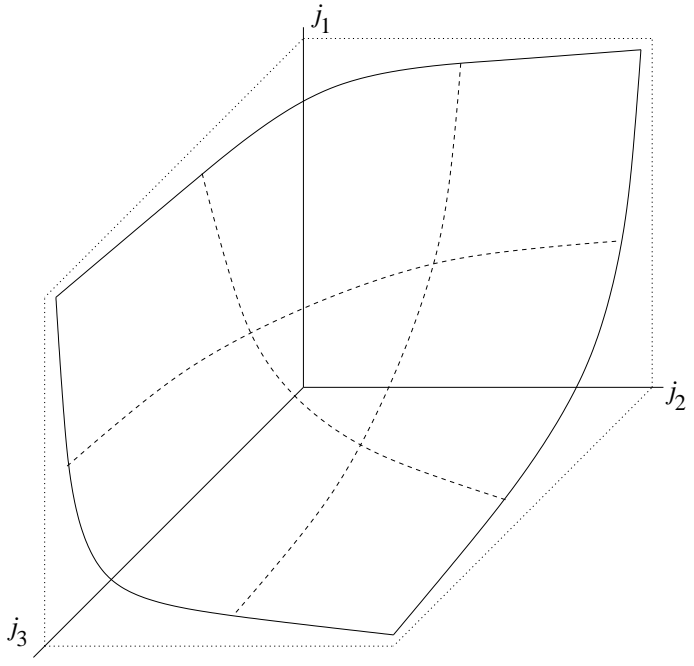

(b)

Figure 3: Phase space of (a) seven-dimensional, and (b) eight-dimensional MP rotating black holes (in a representative quadrant $\left.j_{i} \geq 0\right)$. The surfaces for extremal black holes are represented: black holes exist in the region bounded by these surfaces. (a) $d=7$ : the hyperbolas at which the surface intersects the planes $j_{i}=0$ (which are $j_{k} j_{l}=1 / \sqrt{6}$, i.e., $a_{k} a_{l}=\sqrt{\mu}$ and $r_{0}=0$ ) correspond to naked singularities with zero area; otherwise, the extremal solutions are nonsingular. The three prongs extend to infinity; these are the ultraspinning regimes in which one spin is much larger than the other two. The prong along $j_{i}$ becomes asymptotically of the form $\left|j_{k}\right|+\left|j_{l}\right| \leq f\left(j_{i}\right)$, i.e., the same shape as the five-dimensional diagram in Figure 2(a). (b) $d=8$ : ultraspinning regimes exist in which two spins are much larger than the third one. The sections at large constant $j_{i}$ asymptotically approach the same shape as the six-dimensional phase space Figure 2(b).

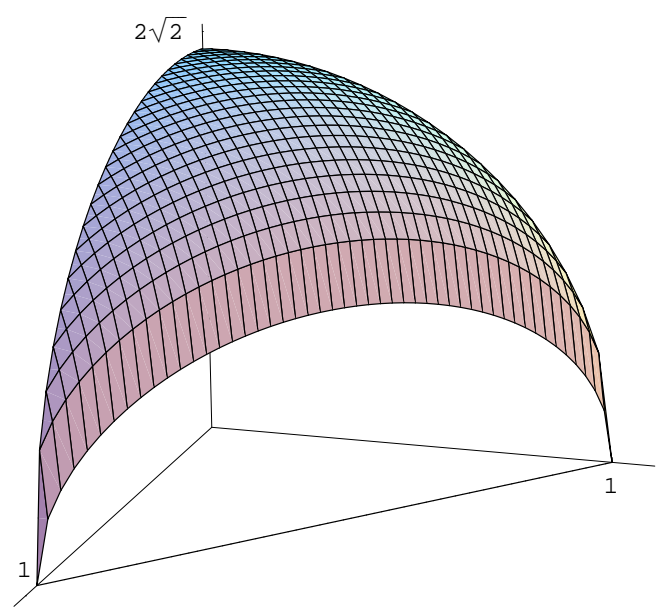

Figure 4: Horizon area $a_{H}\left(j_{1}, j_{2}\right)$ of five-dimensional MP black holes. We only display a representative quadrant $j_{1}, j_{2} \geq 0$ of the full phase space of Figure 2(a), the rest of the surface being obtained by reflection along the planes $j_{1}=0$ and $j_{2}=0$. 


\subsubsection{Global structure}

Let us now discuss briefly the global structure of these solutions, following [200]. The global topology of the solutions outside the event horizon is essentially the same as for the Kerr solution. However, there are cases in which there can be only one nondegenerate horizon: even $d$ with at least one spin vanishing; odd $d$ with at least two spins vanishing; odd $d$ with one $a_{i}=0$ and $\mu>\sum_{i} \Pi_{j \neq i} a_{j}^{2}$ There is also the possibility, for odd $d$ and all nonvanishing spin parameters, of solutions with event horizons with negative $\mu$. However, they contain naked closed causal curves.

The MP solutions have singularities where $\mu r / \Pi F \rightarrow \infty$ for even $d, \mu r^{2} / \Pi F \rightarrow \infty$ for odd $d$. For even $d$ and all spin parameters nonvanishing, the solution has a curvature singularity where $F=0$, which is the boundary of a $(d-2)$-ball at $r=0$, thus generalizing the ring singularity of the Kerr solution; as in the latter, the solution can be extended to negative $r$. If one of the $a_{i}=0$, then $r=0$ itself is singular. For odd $d$ and all $a_{i} \neq 0$, there is no curvature singularity at any $r^{2} \geq 0$. The extension to $r^{2}<0$ contains singularities, though. If one spin parameter vanishes, say $a_{1}=0$, then there is a curvature singularity at the edge of a $(d-3)$-ball at $r=0, \mu_{1}=0$; however, in this case, the ball itself is the locus of a conical singularity. If more than one spin parameter vanishes then $r=0$ is singular. The causal nature of these singularities varies according to the number of horizons that the solution possesses; see [200] for further details.

\subsection{Symmetries}

The Myers-Perry solutions are manifestly invariant under time translations, as well as under the rotations generated by the $N$ Killing vector fields $\partial / \partial \phi_{i}$. These symmetries form a $\mathbb{R} \times U(1)^{N}$ isometry group. In general, this is the full isometry group (up to discrete factors). However, the solutions exhibit symmetry enhancement for special values of the angular momentum. For example, the solution rotating in a single plane (32) has a manifest $\mathbb{R} \times U(1) \times S O(d-3)$ symmetry. If $n$ angular momenta are equal and nonvanishing then the $U(1)^{n}$ associated with the corresponding 2-planes is enhanced to a non-Abelian $U(n)$ symmetry. This reflects the freedom to rotate these 2planes into each other. If $n$ angular momenta vanish then the symmetry enhancement is from $U(1)^{n}$ to an orthogonal group $S O(2 n)$ or $S O(2 n+1)$ for $d$ odd or even respectively [243]. Enhancement of symmetry is reflected in the metric depending on fewer coordinates. For example, in the most extreme case of $N$ equal angular momenta in $2 N+1$ dimensions, the solution has isometry group $\mathbb{R} \times U(N)$ and is cohomogeneity-1, i.e., it depends on a single (radial) coordinate [111, 112].

In addition to isometries, the Kerr solution possesses a "hidden" symmetry associated with the existence of a second-rank Killing tensor, i.e., a symmetric tensor $K_{\mu \nu}$ obeying $K_{(\mu \nu ; \rho)}=$ 0 [245]. This gives rise to an extra constant of motion along geodesics, rendering the geodesic equation integrable. It turns out that the general Myers-Perry solution also possesses hidden symmetries [174, 91] (this was first realized for the special case of $d=5$ [93, 94]). In fact, it has sufficiently many hidden symmetries to render the geodesic equation integrable [207, 173]. In addition, the Klein-Gordon equation governing a free massive scalar field is separable in the Myers-Perry background [90]. These developments have been reviewed in [88].

\subsection{Stability}

The classical linearized stability of these black holes remains largely an open problem. As just mentioned, it is possible to separate variables in the equation governing scalar-field perturbations [147, 26, 195]. However, little progress has been made with the study of linearized gravitational perturbations. For Kerr, the study of gravitational perturbations is analytically tractable because of a seemingly-miraculous decoupling of the components of the equation governing such perturbations, allowing it to be reduced to a single scalar equation [234, 235]. An analogous de- 
coupling has not been achieved for Myers-Perry black holes, except in a particular case that we discuss below.

Nevertheless, it has been possible to infer the appearance of an instability in the ultraspinning regime of black holes in $d \geq 6$ [81]. We have seen that in this regime, when $n$ rotation parameters $a_{i}$ become much larger than the mass parameter $\mu$ and the rest of the $a_{i}$, the geometry of the black-hole horizon flattens out along the fast-rotation planes and approaches a black $2 n$-brane. As discussed in Section 3.4, black p-branes are unstable against developing ripples along their spatial worldvolume directions. Therefore, in the limit of infinite rotation, the MP black holes evolve into unstable configurations. It is then natural to conjecture that the instability already sets in at finite values of the rotation parameters. In fact, the rotation may not need to be too large in order for the instability to appear. The GL instability of a neutral black brane horizon $\mathbb{R}^{p} \times S^{q}$ appears when the size $L$ of the horizon along the brane directions is larger than the size $r_{0}$ of the $S^{q}$. We have seen that the sizes of the horizon along directions parallel and transverse to the rotation plane are $\sim a_{i}$ and $\sim r_{0}$, respectively. This brane-like behavior of MP black holes begins when $a_{i} \gtrsim r_{0}$, which suggests that the instability will appear shortly after crossing thresholds like (39). This idea is supported by the study of the possible fragmentation of the rotating MP black hole: the total horizon area can increase by splitting into smaller black holes whenever $a_{i} \gtrsim r_{0}$ [81]. The analysis of [81] indicates that the instability should be triggered by gravitational perturbations. It is, therefore, not surprising that scalar-field perturbations appear to remain stable even in the ultraspinning regime [26, 195].

This instability has also played a central role in proposals for connecting MP black holes to new black-hole phases in $d \geq 6$. We discuss this in Section 6 .

The one case in which progress has been made with the analytical study of linearized gravitational perturbations is the case of odd dimensionality, $d=2 N+1$, with equal angular momenta $[177,197]$. As discussed above, this Myers-Perry solution is cohomogeneity-1, which implies that the equations governing perturbations of this background are just ODEs. There are two different approaches to this problem, one for $N>1$ [177] and one for $N=1$ (i.e., $d=5$ ) [197].

For $d=5$, the spatial geometry of the horizon is described by a homogeneous metric on $S^{3}$, with $S U(2) \times U(1)$ isometry group. Since $S^{3} \sim S U(2)$, one can define a basis of $S U(2)$ invariant 1-forms and expand the components of the metric perturbation using this basis [197]. The equations governing gravitational perturbations will then reduce to a set of coupled scalar ODEs. These equations have not yet been derived for the Myers-Perry solution (however, this method has been applied to study perturbations of a static Kaluza-Klein black hole with $S U(2) \times U(1)$ symmetry [158]).

For $N>1$, gravitational perturbations can be classified into scalar, vector and tensor types according to how they transform with respect to the $U(N)$ isometry group. The different types of perturbation decouple from each other. Tensor perturbations are governed by a single ODE that is almost identical to that governing a massless scalar field. Numerical studies of this ODE give no sign of any instability [177]. Vector and scalar type perturbations appear to give coupled ODEs; the analysis of these has not yet been completed.

It seems likely that other MP solutions with enhanced symmetry will also lead to more tractable equations for gravitational perturbations. For example, it would be interesting to consider the cases of equal angular momenta in even dimensions (which resemble the Kerr solution in many physical properties), and MP solutions with a single nonzero angular momentum (whose geometry (32) contains a four-dimensional factor, at a constant angle in the $S^{d-4}$, mathematically similar to the Kerr metric; in fact this four-dimensional geometry is type D). The latter case would allow one to test whether the ultraspinning instability is present.

Living Reviews in Relativity

http: //www . livingreviews . org/lrr-2008-6 


\section{$5 \quad$ Vacuum Solutions in Five Dimensions}

In Section 4 we discussed the MP solutions, which can be regarded as higher-dimensional versions of the Kerr solution. However, in recent years it has been realized that higher dimensions allow for a much richer landscape of black-hole solutions that do not have four-dimensional counterparts. In particular, there has been great progress in our understanding of five-dimensional vacuum black holes, insofar as we consider stationary solutions with two rotational Killing vectors. The reason is that this sector of the theory is completely integrable, and solution-generating techniques are available. We begin by analyzing in Section 5.1 a qualitatively new class of solutions with connected horizons: black rings with one and two angular momenta. Then, in Section 5.2, we present a general study of stationary solutions with two rotational symmetries; actually, we can discuss the general case of $d-3$ commuting $U(1)$ spatial isometries. The simplest of these are the generalized Weyl solutions (Section 5.2.1). The general case is addressed in Section 5.2.2, and we discuss the characterization of solutions by their rod structures. The powerful solution-generating technique of Belinsky and Zakharov, based on inverse-scattering methods, is then introduced: the emphasis is on its practical application in generating old and new black-hole solutions. Section 5.3 discusses multiple-black-hole solutions (black Saturns, di-rings and bicycling black rings) obtained in this way. Work towards determining the stability properties of black rings and multiple black holes is reviewed in Section 5.4.

\section{$5.1 \quad$ Black rings}

\subsubsection{One angular momentum}

Five-dimensional black rings are black holes with horizon topology $S^{1} \times S^{2}$ in asymptotically flat spacetime. The $S^{1}$ describes a contractible circle, not stabilized by topology but by the centrifugal force provided by rotation. An exact solution for a black ring with rotation along this $S^{1}$ was presented in [83]. Its most convenient form was given in [79] $\mathrm{as}^{5}$

$$
\begin{aligned}
d s^{2}= & -\frac{F(y)}{F(x)}\left(d t-C R \frac{1+y}{F(y)} d \psi\right)^{2} \\
& +\frac{R^{2}}{(x-y)^{2}} F(x)\left[-\frac{G(y)}{F(y)} d \psi^{2}-\frac{d y^{2}}{G(y)}+\frac{d x^{2}}{G(x)}+\frac{G(x)}{F(x)} d \phi^{2}\right],
\end{aligned}
$$

where

$$
F(\xi)=1+\lambda \xi, \quad G(\xi)=\left(1-\xi^{2}\right)(1+\nu \xi)
$$

and

$$
C=\sqrt{\lambda(\lambda-\nu) \frac{1+\lambda}{1-\lambda}} .
$$

The dimensionless parameters $\lambda$ and $\nu$ must lie in the range

$$
0<\nu \leq \lambda<1
$$

The coordinates vary in the ranges $-\infty \leq y \leq-1$ and $-1 \leq x \leq 1$, with asymptotic infinity recovered as $x \rightarrow y \rightarrow-1$. The axis of rotation around the $\psi$ direction is at $y=-1$, and the axis of rotation around $\phi$ is divided into two pieces: $x=1$ is the disk bounded by the ring, and $x=-1$ is its complement from the ring to infinity. The horizon lies at $y=-1 / \nu$. Outside it, at $y=-1 / \lambda$, lies an ergosurface. A detailed analysis of this solution and its properties can be found in [84] and [76], so we shall only discuss it briefly.

\footnotetext{
${ }^{5}$ An alternative form was found in [140]. The relation between the two is given in [84].
} 
In the form given above, the solution possesses three independent parameters: $\lambda, \nu$, and $R$. Physically, this sounds like one too many: given a ring with mass $M$ and angular momentum $J$, we expect its radius to be dynamically fixed by the balance between the centrifugal and tensional forces. This is also the case for the black ring (50): in general it has a conical defect on the plane of the ring, $x= \pm 1$. In order to avoid it, the angular variables must be identified with periodicity

$$
\Delta \psi=\Delta \phi=4 \pi \frac{\sqrt{F(-1)}}{\left|G^{\prime}(-1)\right|}=2 \pi \frac{\sqrt{1-\lambda}}{1-\nu}
$$

and the two parameters $\lambda, \nu$ must satisfy

$$
\lambda=\frac{2 \nu}{1+\nu^{2}}
$$

This eliminates one parameter, and leaves the expected two-parameter $(\nu, R)$ family of solutions. The mechanical interpretation of this balance of forces for thin rings is discussed in [80]. The Myers-Perry solution with a single rotation is obtained as a limit of the general solution (50) [79], but cannot be recovered if $\lambda$ is eliminated through Equation (55).

The physical parameters of the solution (mass, angular momentum, area, angular velocity, surface gravity) in terms of $\nu$ and $R$ can be found in [84]. It can be seen that while $R$ provides a measure of the radius of the ring's $S^{1}$, the parameter $\nu$ can be interpreted as a 'thickness' parameter characterizing its shape, corresponding roughly to the ratio between the $S^{2}$ and the $S^{1}$ radii.

More precisely, one finds two branches of solutions, whose physical differences are seen most clearly in terms of the dimensionless variables $j$ and $a_{H}$ introduced above. For a black ring in equilibrium, the phase curve $a_{H}(j)$ can be expressed in parametric form as

$$
a_{H}=2 \sqrt{\nu(1-\nu)}, \quad j=\sqrt{\frac{(1+\nu)^{3}}{8 \nu}},
$$

and is depicted in Figure 5.

This curve is easily seen to have a cusp at $\nu=1 / 2$, which corresponds to a minimum value of $j=\sqrt{27 / 32}$ and a maximum $a_{H}=1$. Branching off from this cusp, the thin black-ring solutions $(0<\nu<1 / 2)$ extend to $j \rightarrow \infty$ as $\nu \rightarrow 0$, with asymptotic $a_{H} \rightarrow 0$. The fat black-ring branch $(1 / 2 \leq \nu<1)$ has lower area and extends only to $j \rightarrow 1$, ending at $\nu \rightarrow 1$ at the same zero-area singularity as the MP solution. This implies that in the range $\sqrt{27 / 32} \leq j<1$ there exist three different solutions (thin and fat black rings and MP black holes) with the same value of $j$. The notion of black-hole uniqueness that was proven to hold in four dimensions does not extend to five dimensions.

[76] and [89] contain detailed analyses of the geometrical features of black-ring horizons. Some geodesics of the black-ring metric have been studied with a view towards different applications: [205] studies them in the context of the Penrose process, and [76] considers them for tests of stability. [143] is a more complete analysis of geodesics. 


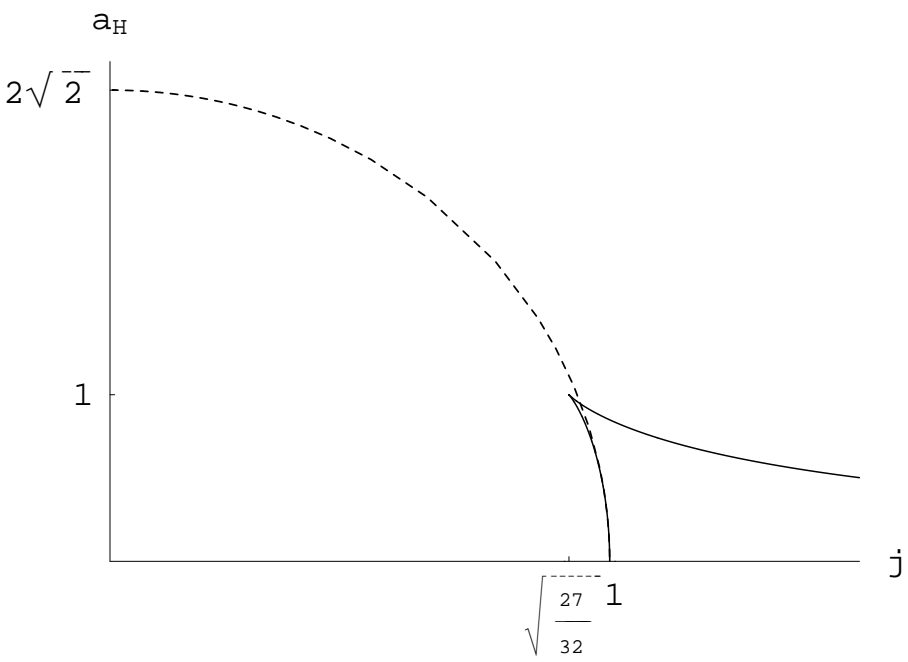

Figure 5: Curve $a_{H}(j)$ of horizon area vs. spin for five-dimensional black rings rotating along their $S^{1}$ (solid). The dashed curve corresponds to five-dimensional MP black holes (see Figure 1). The solid curve for black rings has two branches that meet at a regular, nonextremal minimally-rotating black ring at $j=\sqrt{27 / 32}$ : an upper branch of thin black rings, and a lower branch of fat black rings. Fat black rings always have a smaller area than MP black holes. Their curves meet at the same zero-area naked singularity at $j=1$. 


\subsubsection{Two angular momenta}

Rotation in the second independent plane corresponds to rotation of the $S^{2}$ of the ring. In the limit of infinite $S^{1}$ radius, a section along the length of the ring gives an $S^{2}$ that is essentially like that of a four-dimensional black hole: setting it into rotation is thus similar to having a Kerr-like black hole. Thus, an upper, extremal bound on the rotation of the $S^{2}$ is expected (actually, the motion of the ring along its $S^{1}$ yields a momentum that can be viewed as an electric Kaluza-Klein charge, so instead of a Kerr solution, the $R \rightarrow \infty$ limit yields a rotating electric KK black hole).

Solutions with rotation only along the $S^{2}$, but not on the $S^{1}$, are fairly straightforward to construct and have been given in $[192,87]$. However, these black rings cannot support themselves against the centripetal tension and thus possess conical singularities on the plane of the ring. Constructing the exact solution for a black ring with both rotations is a much more complicated task, which has been achieved by Pomeransky and Sen'kov in [212] (the techniques employed are reviewed in Section 5.2). They have furthermore managed to present it in a fairly compact form:

$$
\begin{aligned}
d s^{2}= & -\frac{H(y, x)}{H(x, y)}(d t+\Omega)^{2}-\frac{F(x, y)}{H(y, x)} d \psi^{2}-2 \frac{J(x, y)}{H(y, x)} d \psi d \phi+\frac{F(y, x)}{H(y, x)} d \phi^{2} \\
& +\frac{2 k^{2} H(x, y)}{(x-y)^{2}(1-\nu)^{2}}\left(\frac{d x^{2}}{G(x)}-\frac{d y^{2}}{G(y)}\right) .
\end{aligned}
$$

Here we follow the notation introduced in [212], except that we have chosen mostly plus signature, and exchanged $\phi \leftrightarrow \psi$ to conform to the notation in Equation (50). The reader should be warned that, although the meanings of $x$ and $y$ are essentially the same in both solutions, the same letters are used in Equation (57) as in Equation (50) for different parameters and functions. In particular, the angles $\phi$ and $\psi$ have been rescaled here to have canonical periodicity $2 \pi$.

The metric functions take a very complicated form in the general case in which the black ring is not in equilibrium (their explicit forms can be found in [196]), but they simplify significantly when a balance of forces (i.e., cancellation of conical singularities) is imposed. In this case the one-form $\Omega$ characterizing the rotation is [212]

$$
\begin{aligned}
\Omega= & -\frac{2 k \lambda \sqrt{(1+\nu)^{2}-\lambda^{2}}}{H(y, x)}\left[\left(1-x^{2}\right) y \sqrt{\nu} d \phi\right. \\
& \left.+\frac{1+y}{1-\lambda+\nu}\left(1+\lambda-\nu+x^{2} y \nu(1-\lambda-\nu)+2 \nu x(1-y)\right) d \psi\right],
\end{aligned}
$$

and the functions $G, H, J$, and $F$ become

$$
\begin{aligned}
G(x)= & \left(1-x^{2}\right)\left(1+\lambda x+\nu x^{2}\right) \\
H(x, y)= & 1+\lambda^{2}-\nu^{2}+2 \lambda \nu\left(1-x^{2}\right) y+2 x \lambda\left(1-y^{2} \nu^{2}\right)+x^{2} y^{2} \nu\left(1-\lambda^{2}-\nu^{2}\right), \\
J(x, y)= & \frac{2 k^{2}\left(1-x^{2}\right)\left(1-y^{2}\right) \lambda \sqrt{\nu}}{(x-y)(1-\nu)^{2}}\left(1+\lambda^{2}-\nu^{2}+2(x+y) \lambda \nu-x y \nu\left(1-\lambda^{2}-\nu^{2}\right)\right), \\
F(x, y)= & \frac{2 k^{2}}{(x-y)^{2}(1-\nu)^{2}}\left[G(x)\left(1-y^{2}\right)\left[\left((1-\nu)^{2}-\lambda^{2}\right)(1+\nu)+y \lambda\left(1-\lambda^{2}+2 \nu-3 \nu^{2}\right)\right]\right. \\
& +G(y)\left[2 \lambda^{2}+x \lambda\left((1-\nu)^{2}+\lambda^{2}\right)+x^{2}\left((1-\nu)^{2}-\lambda^{2}\right)(1+\nu)+x^{3} \lambda\left(1-\lambda^{2}-3 \nu^{2}+2 \nu^{3}\right)\right. \\
& \left.\left.-x^{4}(1-\nu) \nu\left(-1+\lambda^{2}+\nu^{2}\right)\right]\right] .
\end{aligned}
$$

When $\lambda=0$ we find flat spacetime. In order to recover the metric (50) one must take $\nu \rightarrow 0$, identify $R^{2}=2 k^{2}\left(1+\lambda^{2}\right)$ and rename $\lambda \rightarrow \nu$.

The parameters $\nu$ and $\lambda$ are restricted to

$$
0 \leq \nu<1, \quad 2 \sqrt{\nu} \leq \lambda<1+\nu
$$


for the existence of regular black-hole horizons. The bound $\lambda \geq 2 \sqrt{\nu}$ is actually a Kerr-like bound on the rotation of the $S^{2}$. To see this, consider the equation for vanishing $G(y)$,

$$
1+\lambda y+\nu y^{2}=0
$$

which determines the position of the horizon within the allowed range $-\infty<y<-1$. If we identify $y \rightarrow-k / r, \lambda \rightarrow 2 m / k$ and $\nu \rightarrow a^{2} / k^{2}$, this becomes the familiar $r^{2}-2 m r+a^{2}=0$ (this is not to say that $m$ and $a$ correspond to the physical mass and angular momentum parameters, although they are related to them). Requiring the roots of Equation (61) to be real yields the required bound. When it is saturated, $\lambda=2 \sqrt{\nu}$, the horizon is degenerate, and when exceeded, it becomes a naked singularity. The parameter $k$ sets a scale in the solution and gives (roughly) a measure of the ring radius. The extremal Myers-Perry solution is recovered as a limit of the extremal solutions in which $\nu \rightarrow 1, \lambda \rightarrow 2$. However, in order to recover the general Myers-Perry solution as a limit, one needs to relax the equilibrium condition that has been imposed to obtain Equation (59), and use the more general form of these functions given in [196].

The physical parameters $M, J_{\psi}=J_{1}, J_{\phi} \equiv J_{2}$, and $\mathcal{A}_{H}$ of the solution have been computed in [212]. An analysis of the physical properties of the solution, and in particular the phase space, has been presented in [78]. To plot the parameter region where black rings exist, we fix the mass and employ the dimensionless angular-momentum variables $j_{1}$ and $j_{2}$ introduced in Equation (21). The phase space of doubly-spinning black rings is in Figure 6 for the region $j_{1}>j_{2} \geq 0$ (the rest of the plane is obtained by iterating and exchanging $\pm j_{1,2}$ ). It is bounded by three curves (besides the axis, which is not a boundary in the full phase plane):

1. Extremal black rings, with maximal $j_{2}$ for given $j_{1}$, along the curve

$$
j_{1}=\frac{1+4 \sqrt{\nu}+\nu}{4 \nu^{1 / 4}(1+\sqrt{\nu})}, \quad j_{2}=\frac{\nu^{1 / 4}}{2(1+\sqrt{\nu})}, \quad 0 \leq \nu \leq 1
$$

(thin solid curve in Figure 6). This curve extends between $j_{1}=3 / 4, j_{2}=1 / 4($ as $\nu \rightarrow 1$ ) to $j_{1} \rightarrow \infty, j_{2} \rightarrow 0$ (as $\left.\nu \rightarrow 0\right)$. (See [217] for more discussion of extremal rings.)

2. Nonextremal minimally spinning black rings, with minimal $j_{1}$ for given $j_{2}$, along the curve

$$
\begin{aligned}
& j_{1}= \frac{\left(3\left(1+\nu^{2}\right)+(1+\nu) \sqrt{(9+\nu)(1+9 \nu)}-26 \nu\right)^{3 / 2}}{8(1-\nu)^{2} \sqrt{(\sqrt{(9+\nu)(1+9 \nu)}-1-\nu)(5(1+\nu)-\sqrt{(9+\nu)(1+9 \nu)})}}, \\
& j_{2}=\frac{\sqrt{\frac{\nu(3+\sqrt{(9+\nu)(1+9 \nu)}+\nu(18 \sqrt{(9+\nu)(1+9 \nu)}-103+\nu(3 \nu+\sqrt{(9+\nu)(1+9 \nu)}-103))}{\sqrt{(9+\nu)(1+9 \nu)}-1-\nu}}}{2 \sqrt{2}(1-\nu)^{2}}
\end{aligned}
$$

(thick solid curve in Figure 6). This curve extends between $j_{1}=4 / 5, j_{2}=1 / 5($ as $\nu \rightarrow 1)$ and $j_{1}=\sqrt{27 / 32}, j_{2}=0($ as $\nu \rightarrow 0)$.

3. Limiting extremal MP black holes, with $j_{1}+j_{2}=1$ within the range $j_{1} \in[3 / 4,4 / 5]$ (dashed line in Figure 6). There is a discontinuous increase in the area when the black rings reach the extremal MP line.

For doubly-spinning black rings the angular momentum along the $S^{2}$ is always bounded above by the one in the $S^{1}$ as

$$
\left|j_{2}\right|<\frac{1}{3}\left|j_{1}\right|
$$




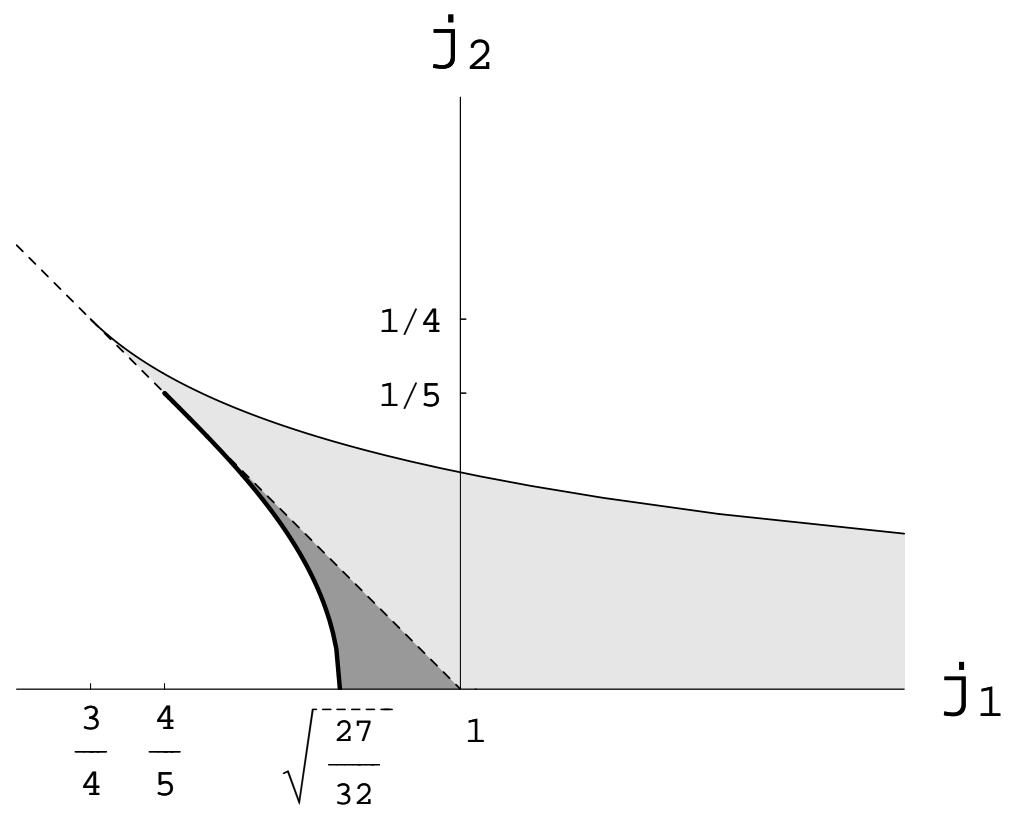

Figure 6: Phase space of doubly-spinning black rings $\left(j_{1} \equiv j_{\psi}, j_{2} \equiv j_{\phi}\right)$, restricted to the representative region $j_{1}>j_{2} \geq 0$. The dashed line $j_{1}+j_{2}=1$ corresponds to extremal MP black holes (see Figure 2(a)). The (upper) thin black curve corresponds to regular extremal black rings with degenerate horizons at maximal $S^{2}$ spin $j_{2}$, for given $S^{1}$ rotation $j_{1}$. It ends on the extremal MP curve at $(3 / 4,1 / 4)$. The (lower) thick black curve corresponds to regular nonextremal black rings with minimal spin $j_{1}$ along $S^{1}$ for given $j_{2}$ on $S^{2}$. It ends on the extremal MP curve at $(4 / 5,1 / 5)$. Black rings exist in the gray-shaded parameter regions bounded by the black curves, the segment $j_{1} \in[3 / 4,4 / 5]$ of the extremal MP dashed line, and the $j_{1}$ axis with $j_{1} \geq \sqrt{27 / 32}$. In the light-gray region there exist only thin black rings. In the dark-gray spandrel between the dashed MP line, the thick black curve, and the axis $\sqrt{27 / 32}<j_{1}<1$, there exist thin and fat black rings and MP black holes: there is discrete three-fold nonuniqueness. 
This is saturated at the endpoint of the extremal black-ring curve $j_{1}=3 / 4, j_{2}=1 / 4$.

Figure 7 shows the phase space covered by all five-dimensional black holes with a single horizon. Two kinds of black rings (thin and fat) and one MP black hole, the three of them with the same values of $\left(M, J_{1}, J_{2}\right)$, exist in small spandrels near the corners of the MP phase-space square. It is curious that, once black rings are included, the available phase space for five-dimensional black holes resembles more closely that of six-dimensional MP black holes, Figure 2(b).

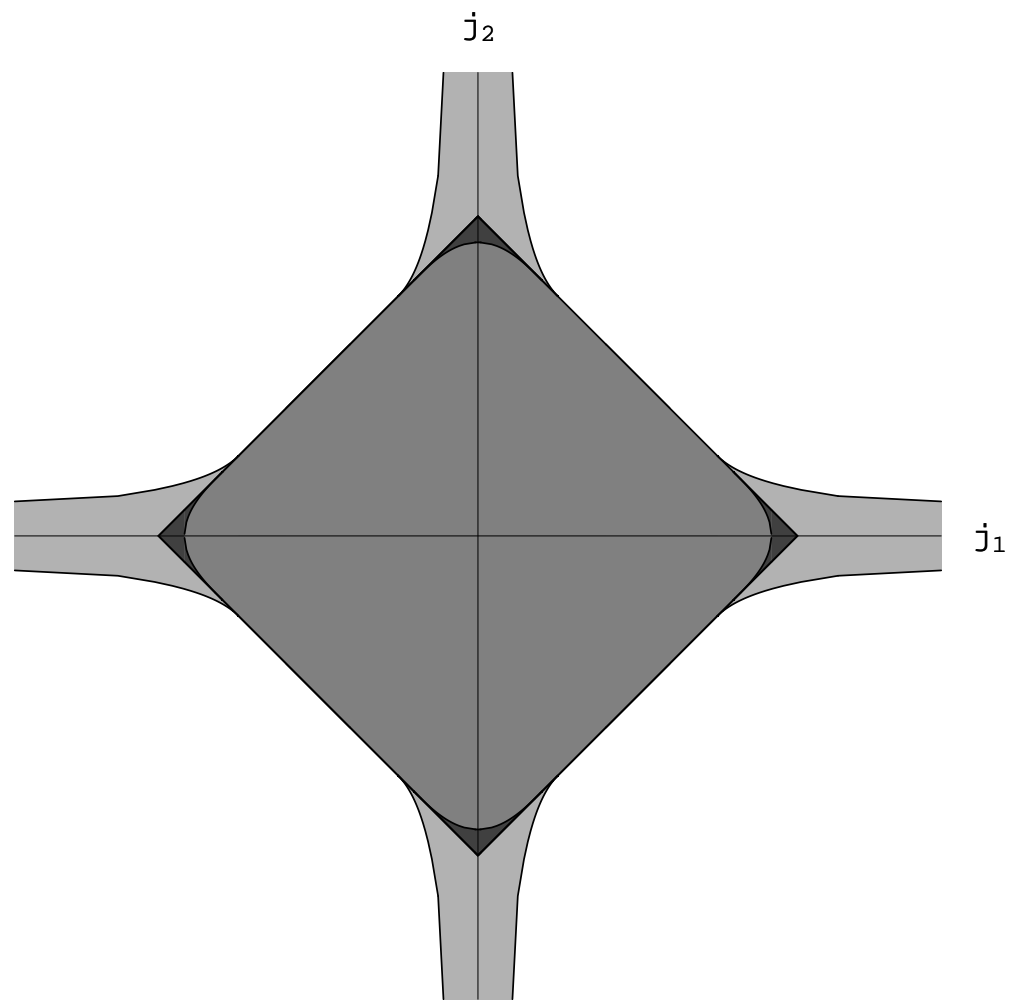

Figure 7: The phase space $\left(j_{1}, j_{2}\right)$ covered by doubly-spinning MP black holes and black rings, obtained by replicating Figure 6 taking $\pm j_{1} \leftrightarrow \pm j_{2}$. The square $\left|j_{1}\right|+\left|j_{2}\right| \leq 1$ corresponds to MP black holes (see Figure 2(a)). The light-gray zones contain thin black rings only, and the medium-gray zone contains MP black holes only. At each point in the dark-gray spandrels near the corners of the square there exist one thin and one fat black ring, and one MP black hole.

[78] contains sectional plots of the surface $a_{H}\left(j_{1}, j_{2}\right)$ for black rings at constant $j_{2}$, for $j_{1}>j_{2} \geq$ 0 , from which it is possible to obtain an idea of the shape of the surface. In the complete range of $j_{1}$ and $j_{2}$ the phase space of five-dimensional black holes (with connected event horizons) consists of the 'dome' of MP black holes (Figure 4 replicated on all four quadrants), with 'romanesque vaults' of black rings protruding from its corners, and additional substructure in the region of nonuniqueness - our knowledge of architecture is insufficient to describe it in words.

It is also interesting to study other properties of black rings, such as temperature and horizon angular velocities, expressions for which can be found in [78]. It is curious to notice that even if the two angular momenta can never be equal, the two angular velocities $\Omega_{1}$ and $\Omega_{2}$ have equal values, for a given mass, when

$$
\lambda=\sqrt{2 \nu-\sqrt{\nu}(1+\nu)},
$$

which lies in the allowed range (60). We can easily understand why this is possible: $\Omega_{1}$ becomes 
arbitrarily small for thin rings, even if $j_{1}$ is large, so it can be made equal to any given $\Omega_{2}$.

On the other hand, the temperature of the black ring - which for thin rings with a single spin is bounded below and diverges as the ring becomes infinitely long and thin (at fixed mass) - decreases to zero when the second spin is taken to the extremal limit, so there exist 'cold' thin black rings.

Some consequences of these features to properties of multiple-ring solutions will be discussed in Section 5.3.

\subsection{Stationary axisymmetric solutions with $d-3$ rotational symmetries}

A sector of five-dimensional vacuum general relativity in which a complete classification of blackhole solutions may soon be achieved is the class of stationary solutions with two angular Killing vectors. Integration of the three Killing directions yields a two-dimensional nonlinear sigma model that is completely integrable. Solutions can be characterized in terms of their rod structure along multiple directions, introduced in [82] and extended in [127]. It has been proven that these data (whose relation to physical parameters is unfortunately not quite direct), in addition to the total mass and angular momenta, uniquely characterize asymptotically-flat solutions [139].

Since most of the analysis is applicable to any number of dimensions, we will keep $d$ arbitrary, although only in $d=4,5$ can the solutions be globally asymptotically flat. So, henceforth, we assume that the spacetime admits $d-2$ commuting, non-null, Killing vectors $\xi_{(a)}=\partial / \partial x^{a}, a=$ $0, \ldots, d-3$ (we assume, although this is not necessary, that the zero-th vector is asymptotically timelike and all other vectors are asymptotically spacelike). Then it is possible to prove that, under natural suitable conditions, the two-dimensional spaces orthogonal to all three Killing vectors are integrable [82]. In this case the metrics admit the form

$$
d s^{2}=g_{a b}(r, z) d x^{a} d x^{b}+e^{2 \nu(r, z)}\left(d r^{2}+d z^{2}\right) .
$$

Without loss of generality we can choose coordinates so that

$$
\operatorname{det} g_{a b}=-r^{2}
$$

For this class of geometries, the Einstein equations divide into two groups, one for the matrix $g$,

$$
\partial_{r} U+\partial_{z} V=0
$$

with

$$
U=r\left(\partial_{r} g\right) g^{-1}, \quad V=r\left(\partial_{z} g\right) g^{-1},
$$

and a second group of equations for $\nu$,

$$
\partial_{r} \nu=-\frac{1}{2 r}+\frac{1}{8 r} \operatorname{Tr}\left(U^{2}-V^{2}\right), \quad \partial_{z} \nu=\frac{1}{4 r} \operatorname{Tr}(U V) .
$$

The equations for $\nu$ satisfy the integrability condition $\partial_{r} \partial_{z} \nu=\partial_{z} \partial_{r} \nu$ as a consequence of Equation (68). Therefore, once $g_{a b}(r, z)$ is determined, the function $\nu(r, z)$ is determined by a line integral, up to an integration constant that can be absorbed by rescaling the coordinates.

Equations (68) and (69) are the equations for the principal chiral field model, a nonlinear sigma model with group $G L(d-2, \mathbb{R})$, which is a completely integrable system. In the present case, it is also subject to the constraint (67). This introduces additional features, some of which will be discussed below.

In order to understand the structure of the solutions of this system, it is convenient to first analyze a simple particular case [82].

Living Reviews in Relativity

http: //www . livingreviews . org/lrr-2008-6 


\subsubsection{Weyl solutions}

Consider the simplest situation in which the Killing vectors are mutually orthogonal. In this case the solutions admit the diagonal form ${ }^{6}$

$$
d s^{2}=-e^{2 U_{0}} d t^{2}+\sum_{a=1}^{d-3} e^{2 U_{a}}\left(d x^{a}\right)^{2}+e^{2 \nu}\left(d r^{2}+d z^{2}\right)
$$

Equations (68) require that $U_{a}(r, z), a=0, \ldots, d-3$, be axisymmetric solutions of the Laplace equation

$$
\left(\partial_{r}^{2}+\frac{1}{r} \partial_{r}+\partial_{z}^{2}\right) U_{a}=0
$$

in the auxiliary three-dimensional flat space

$$
d s^{2}=d z^{2}+d r^{2}+r^{2} d \phi^{2}
$$

while equations $(70)$ for $\nu(r, z)$ become

$$
\begin{aligned}
& \partial_{r} \nu=-\frac{1}{2 r}+\frac{r}{2} \sum_{a=0}^{d-3}\left[\left(\partial_{r} U_{a}\right)-\left(\partial_{z} U_{a}\right)^{2}\right], \\
& \partial_{z} \nu=r \sum_{i=0}^{d-3} \partial_{r} U_{a} \partial_{z} U_{a} .
\end{aligned}
$$

The constraint (67) implies that only $d-3$ of the $U_{a}$ are independent, since they must satisfy

$$
\sum_{a=0}^{d-3} U_{a}=\log r
$$

Thus we see that solutions are fully determined once the boundary conditions for the $U_{i}$ are specified at infinity and at the $z$-axis. Note that $\log r$ is the solution that corresponds to an infinite rod of zero thickness and linear mass density $1 / 2$ along the axis $r=0$. The solutions are in fact characterized by the 'rod' sources of $U_{i}$ along the axis, which have to add up to an infinite rod (76). The potential for a semi-infinite rod along $\left[a_{k},+\infty\right)$ with linear density $\varrho$ is

$$
U=\varrho \log \mu_{k},
$$

where

$$
\mu_{k}=\sqrt{r^{2}+\left(z-a_{k}\right)^{2}}-\left(z-a_{k}\right) .
$$

If the rod instead extends along $\left(-\infty, a_{k}\right]$ then

$$
U=\varrho \log \bar{\mu}_{k},
$$

where

$$
\bar{\mu}_{k}=-\sqrt{r^{2}+\left(z-a_{k}\right)^{2}}-\left(z-a_{k}\right)=-\frac{r^{2}}{\mu_{k}} .
$$

Given the linearity of equations (72), one can immediately construct the potential for a finite rod of density $\varrho$ along $\left[a_{k-1}, a_{k}\right]$ as

$$
U=\varrho \log \left(\frac{\mu_{k-1}}{\mu_{k}}\right) .
$$

\footnotetext{
${ }^{6}$ An equivalent system, but with a cosmological interpretation under a Wick rotation of the coordinates $(t, r, z) \rightarrow$ $(x, t, \tilde{z})$, is discussed in [86], along with some simple solution-generating techniques.
} 
The functions $U_{i}$ for any choice of rods are sums of these. Integration of Equation (74) is then a straightforward, if tedious, matter; see appendix G in [82]. [165] has applied the inverse scattering method (to be reviewed below) to provide explicit diagonal solutions with an arbitrary number of rods.

At a rod source for $U_{i}$, the orbits of the corresponding Killing vector vanish: if it is an angular Killing vector $\partial_{\phi_{i}}$, then the corresponding one-cycles shrink to zero size, and the periodicity of $\phi_{i}$ must be chosen appropriately in order to avoid conical singularities; if it is instead the timelike Killing $\partial_{t}$, then it becomes null there. In both cases, a necessary (but not sufficient) condition for regularity at the rod is that the linear density be

$$
\varrho=\frac{1}{2}
$$

Otherwise when $r \rightarrow 0$ at the rod, the curvature diverges. If all rods are of density $1 / 2$, then given the constraint (76), at any given point on the axis there will be one cycle of zero (or null) length with all others having finite size. This phenomenon of some cycles smoothly shrinking to zero with other cycles blowing up to finite size as one moves along the axis (essentially discovered by Weyl in 1917 [247]), is referred to in string-theoretical contexts as 'bubbling'. When it is $\partial_{t}$ that becomes null, the rod corresponds to a horizon.

The rod structures for the four and five-dimensional Schwarzschild and Tangherlini solutions are depicted in Figure 8. The Rindler space of uniformly-accelerated observers is recovered as the horizon rod becomes semi-infinite, $a_{2} \rightarrow \infty$. [82] gives a number of 'rules of thumb' for interpreting rod diagrams.

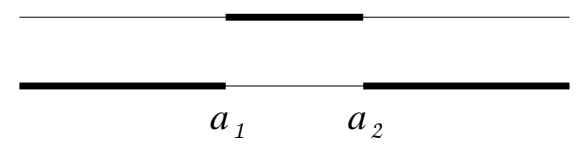

(a)

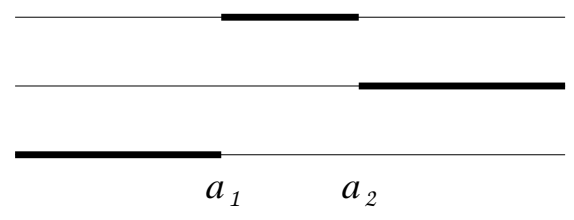

(b)

Figure 8: Rod structures for the (a) 4D Schwarzschild and (b) 5D Tangherlini black holes. From top to bottom, the lines represent the sources for the time, $\phi$ and $\psi$ (in $5 \mathrm{D}$ ) potentials $U_{t}, U_{\phi}, U_{\psi}$.

\subsubsection{General axisymmetric class}

In the general case where the Killing vectors are not orthogonal to each other, the simple construction in terms of solutions of the linear Laplace equation does not apply. Nevertheless, the equations can still be completely integrated, and the characterization of solutions in terms of rod structure can be generalized.

5.2.2.1 Rod structure and regularity Let us begin by extending the characterization of rods [127]. In general, a rod is an interval along the $z$-axis, where the action of a Killing vector $v$ has fixed points (for a spacelike vector) or it becomes null (for a timelike vector). In both cases $|v|^{2} \rightarrow 0$ at the rod. In general, $v$ will be a linear combination $v=v^{a} \xi_{(a)}$ in a given Killing basis $\xi_{(a)}, a=0, \ldots, d-3$; usually, this basis is chosen so that it becomes a coordinate basis of orthogonal vectors at asymptotic infinity. In the orthogonal case of the previous section 5.2.1 one could assign a basis vector (and only one, for rod density 1/2) $\xi_{(a)}$ to each rod, but this is not possible, in general, if the vectors are not orthogonal. For instance, at a rod corresponding to a rotating horizon, the Killing vector that vanishes is typically of the form $\partial_{t}+\Omega_{a} \partial_{\phi_{a}}$. 
More precisely, the condition (67) implies that the matrix $g(r=0, z)$ must have at least one zero eigenvalue. It can be shown that regularity of the solution (analogous to the requirement of density $1 / 2$ in the orthogonal case) requires that only one eigenvalue is zero at any given interval on the axis [127]. Each such interval is called a rod, and, for each $\operatorname{rod} z \in[a, b]$, we assign a direction vector $v$ such that

$$
g(0, z) v=0 \quad \text { for } \quad z \in[a, b] .
$$

At isolated points on the axis the kernel of $g(r=0, z)$ is spanned by two vectors instead of only one: this will happen at the points where two intervals with different eigenvectors meet. The rod structure of the solution consists of the specification of intervals $a_{1}<a_{2}<\cdots<a_{n}$, plus the $n+1$ directions $v_{(k)} \in \operatorname{ker}(g(0, z))$ for each $\operatorname{rod}\left[a_{k-1}, a_{k}\right], k=1, \ldots, n+1$ (with $a_{0}=-\infty$, $\left.a_{n+1}=+\infty\right)$ [127]. The vector $v$ is defined up to an arbitrary normalization constant.

The rod is referred to as timelike or spacelike according to the character of the rescaled norm $g_{a b}(r, z) v^{a} v^{b} / r^{2}$ at the $\operatorname{rod} r \rightarrow 0, z \in\left[a_{k-1}, a_{k}\right]$. For a timelike rod normalized such that the generator of asymptotic time translations enters with coefficient equal to one, the rest of the coefficients correspond to the angular velocities of the horizon (if this satisfies all other regularity requirements).

For a spacelike rod, the following two regularity requirements are important. First, given a rod-direction vector

$$
v=\frac{\partial}{\partial \psi}
$$

with norm

$$
|v|^{2}=g_{i j}(r, z) v^{i} v^{j}
$$

the length $L=|v| \Delta \psi$ of its circular orbits at constant $r, z$, vanishes near the rod like $\sim r$. Since the proper radius of these circles approaches $e^{\nu(0, z)} d r$, a conical singularity will be present at the rod unless these orbits are periodically identified with period

$$
\Delta \psi=2 \pi e^{\nu(0, z)}\left(\frac{\partial|v|}{\partial r}(0, z)\right)^{-1} .
$$

When several rods are present, it may be impossible to satisfy simultaneously all the periodicity conditions. The physical interpretation is that the forces among objects in the configuration cannot be balanced and as a result, conical singularities appear. If the geometry admits a Wick rotation to Euclidean time, then Equation (86) gives the temperature $T_{H}=2 \pi / \Delta \psi$ of the horizon associated with the rod.

Second, the presence of time components on a spacelike rod creates causal pathologies. Consider a vector $\zeta$ that is timelike in some region of spacetime, and whose norm does not vanish at a given spacelike rod $[a, b]$. If the direction vector $v$ associated with this rod is such that

$$
v \cdot \zeta \neq 0 \quad \text { at } \quad r=0, z \in[a, b]
$$

then making the orbits of $v$ periodic introduces closed timelike curves; periodicity $\Delta \psi$ along orbits of $v$ requires that the orbits of $\zeta$ be identified as well with periods equal to an integer fraction of $(v \cdot \zeta) \Delta \psi$. Then, closed timelike curves will appear wherever $\zeta$ is timelike. These are usually regarded as pathological if they occur outside the horizon, so such time components on spacelike rods must be avoided.

Further analysis of Equations (68), (70) and their source terms can be found in [130].

5.2.2.2 The method of Belinsky and Zakharov As shown by Belinsky and Zakharov (BZ), the system of nonlinear equations (67) and (68) is completely integrable [11, 10]. It admits a Lax pair of linear equations (spectral equations) whose compatibility conditions coincide with the 
original nonlinear system. This allows one to generate an infinite number of solutions, starting from known ones, following a purely algebraic procedure. Since, as we have seen, we can very easily generate diagonal solutions, this method allows us to construct a vast class of axisymmetric solutions. It seems likely, but to our knowledge has not been proven, that all axisymmetric solutions can be generated in this way.

The spectral versions of Equations (67) and (68) are

$$
D_{1} \Psi=\frac{r V-\lambda U}{\lambda^{2}+r^{2}} \Psi, \quad D_{2} \Psi=\frac{r U+\lambda V}{\lambda^{2}+r^{2}} \Psi
$$

where $\lambda$ is the (in general complex) spectral parameter, independent of $r$ and $z$, and $D_{1}$ and $D_{2}$ are two commuting differential operators,

$$
D_{1}=\partial_{z}-\frac{2 \lambda^{2}}{\lambda^{2}+r^{2}} \partial_{\lambda}, \quad D_{2}=\partial_{r}+\frac{2 \lambda r}{\lambda^{2}+r^{2}} \partial_{\lambda} .
$$

The function $\Psi(\lambda, r, z)$ is a $(d-2) \times(d-2)$ matrix such that $\Psi(0, r, z)=g(r, z)$, where $g$ is a solution of Equation (68). Compatibility of Equations (88) then implies Equations (68) and (69).

Since Equations (88) are linear, we can construct new solutions by 'dressing' a 'seed' solution $g_{0}$. The seed defines matrices $U_{0}$ and $V_{0}$ through Equations (69). Equations (88) can then be solved to determine $\Psi_{0}$. Then, we 'dress' this solution using a matrix $\chi=\chi(\lambda, r, z)$ to find a new solution of the form

$$
\Psi=\chi \Psi_{0}
$$

Introducing Equation (90) into Equation (88) we find a system of equations for $\chi$. The simplest and most interesting solutions are the solitonic ones, for which the matrix $\chi(\lambda, r, z)$ can be written in terms of simple poles

$$
\chi=1+\sum_{k=1}^{n} \frac{R_{k}}{\lambda-\tilde{\mu}_{k}} .
$$

The residue matrices $R_{k}$ and the 'pole-position' functions $\tilde{\mu}_{k}$ depend only on $r$ and $z$. For a dressing function of this form, it is fairly straightforward to determine the functions $\tilde{\mu}_{k}$ and the matrices $R_{k}$. The pole positions are

$$
\tilde{\mu}_{k}=\left\{\begin{array}{l}
\mu_{k} \text { for a soliton, } \\
\bar{\mu}_{k} \text { for an antisoliton, }
\end{array}\right.
$$

where $\mu_{k}, \bar{\mu}_{k}$ were introduced in Equations (78) and (80). In principle the parameters $a_{k}$ may be complex and must appear in conjugate pairs, if the metric is to be real. However, in all the examples that we consider, the $a_{k}$ are real; complex poles appear to lead to naked singularities, for instance, in the Kerr solution they occur when the extremality bound on the angular momentum is violated.

The solution for the matrices $R_{k}$, where $k$ labels the solitons, can be constructed by first introducing a set of $d-2$-dimensional vectors $m^{(k)}$ using the seed as

$$
m_{a}^{(k)}=m_{0 b}^{(k)}\left[\Psi_{0}^{-1}\left(\tilde{\mu}_{k}, r, z\right)\right]_{b a} .
$$

The constant vectors $m_{0}^{(k)}$ introduced here are the crucial new data determining the rod orientations in the new solution. Now defining the symmetric matrix

$$
\Gamma_{k l}=\frac{m_{a}^{(k)}\left(g_{0}\right)_{a b} m_{b}^{(l)}}{r^{2}+\tilde{\mu}_{k} \tilde{\mu}_{l}}
$$


the $R_{k}$ are

$$
\left(R_{k}\right)_{a b}=m_{a}^{(k)} \sum_{l=1}^{n} \frac{\left(\Gamma^{-1}\right)_{l k} m_{c}^{(l)}\left(g_{0}\right)_{c b}}{\tilde{\mu}_{l}} .
$$

All the information about the solitons that are added to the solution is contained in the soliton positions $a_{k}$ and the soliton-orientation vectors $m_{0}^{(k)}$. This is all we need to determine the dressing matrix in Equation (91), and then the new metric $g(r, z)=\Psi(0, r, z)=\chi(0, r, z) \Psi_{0}(0, r, z)$,

$$
g_{a b}=\left(g_{0}\right)_{a b}-\sum_{k, l=1}^{n} \frac{\left(g_{0}\right)_{a c} m_{c}^{(k)}\left(\Gamma^{-1}\right)_{k l} m_{d}^{(l)}\left(g_{0}\right)_{d b}}{\tilde{\mu}_{k} \tilde{\mu}_{l}} .
$$

There is, however, one problem that turns out to be particularly vexing in $d>4$ : the new metric $g$ in Equation (96) does not satisfy, in general, the constraint (67); the introduction of the $n$ solitons gives a determinant for the new metric

$$
\operatorname{det} g=(-1)^{n} r^{2 n}\left(\prod_{k=1}^{n} \tilde{\mu}_{k}^{-2}\right) \operatorname{det} g_{0}
$$

A determinant of a new physical solution $g^{\text {(phys) }}$ must satisfy constraint (67), but $g$ in Equation (97) does not. This problem can be expediently resolved by simply multiplying the metric obtained in Equation (96) by an overall factor

$$
g^{\text {(phys) }}= \pm\left(\frac{r^{2}}{ \pm \operatorname{det} g}\right)^{\frac{1}{d-2}} g
$$

(we may ignore here the choice of \pm signs). Now, observe that the problem that Equation (98) solves is that of making the rod densities at each point along the axis to add up to a total density of $1 / 2$. However, recall that regularity required that individual rod densities, and not just their sums, be exactly equal to $1 / 2$. This is a problem for Equation (98) whenever $d>4$; since Equation (97) contains only solitons and antisolitons with regular densities $\pm 1 / 2$ (with $-1 / 2$ allowed only at intermediate steps), the fractional power in Equation (98) introduces rods with fractional densities $1 /(d-2)$, which in $d>4$ will always result in curvature singularities at the rod.

A possible way out of this problem is to restrict oneself to transformations that act only on a $2 \times 2$ block of the seed $g_{0}$ [165]. In this case it is possible to apply the above renormalization to only this part of the metric - effectively, the same as in four dimensions - and thus obtain a solution with the correct, physical rod densities. However, it is clear that, if we start from diagonal seeds, this method cannot deal with solutions with off-diagonal terms in more than one $2 \times 2$ block, e.g., with a single rotation. It cannot be applied to obtain solutions with rotation in several planes. Still, [7] applies this method to obtain solutions with arbitrary number of rods, with rotation in a single plane.

Fortunately, a clever and very practical way out of this problem has been proposed by Pomeransky [211], that can deal with the general case in any number of dimensions. The key idea is the observation that Equation (97) is independent of the 'realignment' vectors $m_{0 b}^{(k)}$. One may then start from a solution with physical rod densities, 'remove' a number of solitons from it (i.e., add solitons or antisolitons with negative densities $-1 / 2$ ), and then re-add these same solitons, but now with different vectors $m_{0 b}^{(k)}$, so the rods affected by these solitons acquire, in general, new directions. If the original seed solution satisfied the determinant constraint (67), then by construction so will the metric obtained after re-adding the solitons (including, in particular, the sign). And more importantly, if the densities of the initial rods are all $\pm 1 / 2$ and negative densities do not appear in the end result, the final metric will only contain regular $1 / 2$ densities. 
In the simplest form of this method, one starts from a diagonal, hence static, solution $\left(g_{0}, e^{2 \nu_{0}}\right)$ and then removes some solitons or antisolitons with 'trivial' vectors aligned with one of the Killing basis vectors $m_{0}^{(k)}=\xi_{(a)}$, i.e., $m_{0 b}^{(k)}=\delta_{a b}$ (recall that in the diagonal case this alignment of rods is indeed possible). Removing a soliton or antisoliton $\tilde{\mu}_{k}$ at $z=a_{k}$ aligned with the direction $a$ amounts to changing

$$
\left(g_{0}^{\prime}\right)_{a a}=-\frac{\tilde{\mu}_{k}^{2}}{r^{2}}\left(g_{0}\right)_{a a}
$$

while leaving unchanged all other metric components $\left(g_{0}^{\prime}\right)_{b b}=\left(g_{0}\right)_{b b}$ with $b \neq a$. We now take this new metric $g_{0}^{\prime}$ as the seed to which, following the BZ method, we re-add the same solitons and antisolitons, but with more general vectors $m_{0}^{(k)}=\sum_{b} C_{b} \xi_{(b)}$. Note that there is always the freedom to re-scale each of these vectors by a constant. Finally, the two-dimensional conformal factor $e^{2 \nu}$ for the new solution is obtained from the seed as

$$
e^{2 \nu}=e^{2 \nu_{0}} \frac{\operatorname{det} \Gamma}{\operatorname{det} \Gamma_{0}},
$$

where the matrices $\Gamma_{0}$ and $\Gamma$ are obtained from Equation (94) using $g_{0}$ and $g$ respectively.

If the vectors $m_{0 a}^{(k)}$ for the re-added solitons mix the time and spatial Killing directions, then this procedure may yield a stationary (rotating) version of the initial static solution. The method requires the determination of the function $\Psi_{0}(\lambda, r, z)$ that solves Equation (88) for the seed. This is straightforward to determine for diagonal seeds (see the examples below), so for these the method is completely algebraic. Although even a two-soliton transformation of a multiple-rod metric can easily result in long expressions for the metric coefficients, the method can be readily implemented in a computer program for symbolic manipulation. The procedure can also be applied, although it becomes quite more complicated, to nondiagonal seeds. In this case, the function $\Psi_{0}(\lambda, r, z)$ for the nondiagonal seed is most simply determined if this solution itself is constructed starting from a diagonal seed. The doubly-spinning black ring of [212] was obtained in this manner.

5.2.2.3 BZ construction of the Kerr, Myers-Perry and black-ring solutions We sketch here the method to obtain all known black-hole solutions with connected horizons in four and five dimensions. These solutions illustrate how to add rotation to $S^{2}, S^{3}$ and $S^{1} \times S^{2}$ components of multiple-black-hole horizons.

The simplest case, which demonstrates one of the basic tools for adding rotation in more complicated cases, is the Kerr solution - in fact, one generates the Kerr-Taub-NUT solution, and then sets the nut charge to zero. Begin from the Schwarzschild solution, generated, e.g., using the techniques available for Weyl solutions [82], and whose rod structure is depicted in Figure 8. The seed metric is

$$
g_{0}=\operatorname{diag}\left\{-\frac{\mu_{1}}{\mu_{2}}, r^{2} \frac{\mu_{2}}{\mu_{1}}\right\},
$$

with $a_{1}<a_{2}$. Then, remove an antisoliton at $z=a_{1}$, with vector $m_{0}^{(1)}=(1,0)$, and a soliton at $z=a_{2}$ with the same vector. Following rule (99) we obtain the matrix

$$
g_{0}^{\prime}=\operatorname{diag}\left\{-\frac{\mu_{2}}{\mu_{1}}, r^{2} \frac{\mu_{2}}{\mu_{1}}\right\} .
$$

It is now convenient (but not necessary) to re-scale the metric by a factor $\mu_{1} / \mu_{2}$. This yields the new metric

$$
\tilde{g}_{0}^{\prime}=\operatorname{diag}\left\{-1, r^{2}\right\} .
$$

This is nothing but flat space; what we have done here is undo the generation of the Schwarzschild metric out of Minkowski space. But now the idea is to retrace our previous steps, after re-adding the solitons with new vectors $m_{0}^{(1,2)}$. 
So, following the method above, add an antisoliton at $z=a_{1}$ and a soliton at $z=a_{2}$, with respective constant vectors $m_{0}^{(1)}=\left(1, A_{1}\right)$ and $m_{0}^{(2)}=\left(1, A_{2}\right)$. For this step, we need to construct the matrix $\Gamma$ in Equation (94), which in turn requires the matrix

$$
\tilde{\Psi}_{0}(\lambda, r, z)=\operatorname{diag}\left\{-1, r^{2}-2 z \lambda-\lambda^{2}\right\}
$$

that solves the spectral Equations (88) for $\tilde{g}_{0}^{\prime}$. Equations (93), (94), and (96) then give a new metric $\tilde{g}$. But we still have to undo the rescaling we did to get $\tilde{g}_{0}^{\prime}$ from $g_{0}^{\prime}$. That is,

$$
g=\frac{\mu_{2}}{\mu_{1}} \tilde{g}
$$

By construction, $g$ is correctly normalized, i.e., it satisfies Equation (67). Finally, the function $e^{2 \nu}$ is obtained using Equation (100), which is straightforward since we obtained $\Gamma$ when the solitons were re-added, and $\Gamma_{0}=\Gamma\left(A_{1}=A_{2}=0\right)$. The new solution contains two more parameters than the Schwarzschild seed: these correspond to the rotation and nut parameters. The latter can be set to zero once the parameters are correctly identified. For details about how the Boyer-Lindquist form of the Kerr solution is recovered, see [10].

The Myers-Perry black hole with two angular momenta is obtained in a very similar way [211] starting from the five-dimensional Schwarzschild-Tangherlini solution, whose rod structure is shown in Figure 8. We immediately see that

$$
g_{0}=\operatorname{diag}\left\{-\frac{\mu_{1}}{\mu_{2}}, \mu_{2}, \frac{r^{2}}{\mu_{1}}\right\} .
$$

Now remove an antisoliton at $z=a_{1}$ and a soliton at $z=a_{2}$, both with vectors $(1,0,0)$, to find

$$
g_{0}^{\prime}=\operatorname{diag}\left\{-\frac{\mu_{2}}{\mu_{1}}, \mu_{2}, \frac{r^{2}}{\mu_{1}}\right\} .
$$

An overall rescaling by $\mu_{1} / \mu_{2}$ simplifies the metric to

$$
\tilde{g}_{0}^{\prime}=\operatorname{diag}\left\{-1, \mu_{1}, \frac{r^{2}}{\mu_{2}}\right\}=\operatorname{diag}\left\{-1, \mu_{1},-\bar{\mu}_{2}\right\} .
$$

This is the metric that we dress with solitons applying the BZ method. Observe that it does not satisfy Equation (67) and so it is not a physical metric, but this is not a problem. The associated $\tilde{\Psi}_{0}$ is

$$
\tilde{\Psi}_{0}(\lambda, r, z)=\operatorname{diag}\left\{-1, \mu_{1}-\lambda,-\bar{\mu}_{2}+\lambda\right\} .
$$

Now add the antisoliton at $z=a_{1}$ and the soliton at $z=a_{2}$, with vectors $m_{0}^{(1)}=\left(1,0, B_{1}\right)$ and $m_{0}^{(2)}=\left(1, A_{2}, 0\right)$ (more general vectors give singular solutions). A final rescaling of the metric by $\mu_{2} / \mu_{1}$ yields a physically-normalized solution. The two new parameters that we have added are associated with the two angular momenta. The MP solution with a single spin can be obtained through a one-soliton transformation, which is not possible for Kerr. See [211] for the transformation to the coordinates used in Section 4.2.

The black ring with rotation along the $S^{1}$ requires a more complicated seed, but, on the other hand, it requires only a one-soliton transformation [77] (the first systematic derivations of this solution used a two-soliton transformation [148, 240]). The seed is described in Figure 9. The static black ring of [82] (which necessarily contains a conical singularity) is recovered for $a_{1}=a_{2}$. However, one needs to introduce a 'phantom' soliton point at $a_{1}$ and a negative density rod, in order to eventually obtain the rotating black ring. Thus, we see that the initial solution need not satisfy any regularity requirements. To obtain the rotating black ring, we remove an antisoliton 


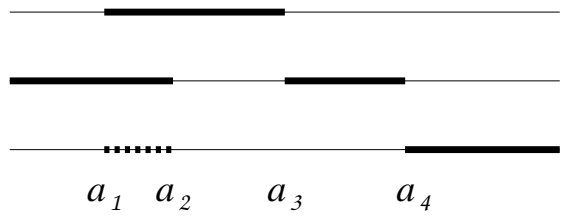

(a)

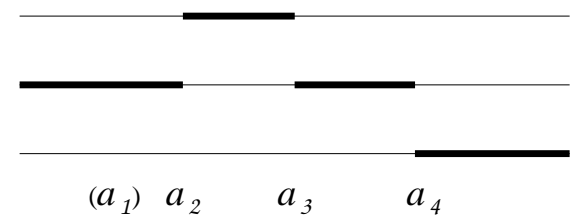

(b)

Figure 9: Rod structures for (a) the seed used to generate (b) the rotating black ring. The seed metric is diagonal, and the dotted rod has negative density $-1 / 2$. In the final solution the parameters can be adjusted so that the metric at $z=a_{1}$ on the axis is completely smooth. The (upper) horizon rod in the final solution has mixed direction $\left(1,0, \Omega_{\psi}\right)$, while the other rods are aligned purely along the $\phi$ or $\psi$ directions.

at $z=a_{1}$ with direction $(1,0,0)$, and re-add it with vector $m_{0}^{(1)}=\left(1,0, B_{1}\right)$. At the end of the process one must adjust the parameters, including $B_{1}$ and the rod positions, to remove a possible singularity at the phantom point $a_{1}$.

The doubly-spinning ring has resisted all attempts at deriving it directly from a diagonal, static seed. Instead, [212] obtained it in a two-step process. Rotation of the $S^{2}$ of the ring is similar to the rotation of the Kerr solution. In fact, the black-ring solutions with rotation only along the $S^{2}$ can be obtained by applying to the static black ring the same kind of two-soliton transformations that yielded Kerr from a Schwarzschild seed (101) [239]. Hence, if we begin from the black ring rotating along the $S^{1}$ and perform similar soliton and antisoliton transformations at the endpoints of the horizon rod, we can expect to find a doubly-spinning ring. The main technical difficulty is in constructing the function $\Psi_{0}(\lambda, r, z)$ for the single-spin black-ring solution (50). However, if we construct this solution via a one-soliton transformation as we have just explained, this function is directly obtained from Equation (90). In this manner, solution (57) was derived.

This method has also been applied to construct solutions with disconnected components of the horizon, which we shall discuss next. The previous examples provide several 'rules of thumb' for constructing such solutions. However, there is no precise recipe for the most efficient way of generating the sought solution. Quite often, unexpected pathologies show up, of both local and global type, so a careful analysis of the solutions generated through this method is always necessary.

Finally, observe that there are certain arbitrary choices in this method; it is possible to choose different solitons and antisolitons, with different orientations, and still get essentially the same final physical solution. Also, the intermediate rescaling, and the form for $\tilde{\Psi}_{0}$, admit different choices. All this may lead to different-looking forms of the final solution, some of them possibly simpler than others. Occasionally, spurious singularities may be introduced through bad choices.

5.2.2.4 Other methods In four dimensions there exists another technique, akin to the Bäcklund transformation that adds solitons to a seed solution, to integrate the equations for the stationary axisymmetric class of vacuum solutions [203, 137, 29]. [192, 149] have extended this to higher dimensions. Unfortunately, though this method can produce simpler expressions than the BZ technique, it cannot deal with more than two off-diagonal terms, and hence, no more than a single angular momentum. [192] applied this method to derive a black ring with rotation in the $S^{2}$ (but not along the $S^{1}$ ). The same authors used this technique to provide the first systematic derivation (i.e., through explicit integration of Einstein's equations, instead of guesswork) of the black ring with rotation along $S^{1}$ [148]. The connection between the Bäcklund transformation method and the BZ technique has been studied in [238], where it is argued that all the solutions obtained by a two-soliton Bäcklund transformation on an arbitrary diagonal seed are contained among those 
that the BZ method generates from the same seed. This is not too surprising in view of similar, and more general, results in four dimensions [53, 54]. It may be interesting to investigate the application of related but more efficient, axisymmetric solution-generating methods [226, 189] to higher dimensions.

[114] develops a different algebraic method to obtain stationary axisymmetric solutions in five dimensions from a given seed. An $S O(2,1)$ subgroup of the "hidden" $S L(3, \mathbb{R})$ symmetry of solutions with at least one spatial Killing vector (the presence of a second one is assumed later) is identified that preserves the asymptotic boundary conditions, and whose action on a static solution generates a one-parameter family of stationary solutions with angular momentum, e.g., one can obtain the Myers-Perry solution from a Schwarzschild-Tangherlini seed. It is conjectured that all vacuum stationary axisymmetric solutions can be obtained by repeated application of these transformations on static seeds.

\subsection{Multiple-black-hole solutions}

In $d=4$ it is believed that there are no stationary multiple-black-hole solutions of vacuum gravity. However, such solutions do exist in $d=5$. 'Black Saturn' solutions, in which a central MP-type of black hole is surrounded by a concentric rotating black ring, have been constructed in [77]. They exhibit a number of interesting features, such as rotational dragging of one black object by the other, as well as both co- and counter-rotation. For instance, we may start from a static seed and act with the kind of one-soliton BZ transformation that turns on the rotation of the black ring. This gives angular momentum (measured by a Komar integral on the horizon) to the black ring but not to the central black hole. However, the horizon rod of this black hole is reoriented and acquires a nonzero angular velocity: the black hole is dragged along by the black-ring rotation. It is also possible (this needs an additional one-soliton transformation that turns on the rotation of the MP black hole) to have a central black hole with a static horizon that nevertheless has nonvanishing angular momentum; the 'proper' inner rotation of the black hole is cancelled at its horizon by the black-ring drag force.

The explicit solutions are rather complicated, but an intuitive discussion of their properties is presented in [73]. The existence of black Saturns is hardly surprising; since black rings can have arbitrarily large radius, it is clear that we can put a small black hole at the center of a very long black ring, and the interaction between the two objects will be negligible. In fact, since a black ring can be made arbitrarily thin and light for any fixed value of its angular momentum, for any nonzero values of the total mass and angular momentum, we can obtain a configuration with larger total area than any MP black hole or black ring; put almost all the mass in a central, almost-static black hole, and the angular momentum in a very thin and long black ring. Such configurations can be argued to attain the maximal area (i.e., entropy) for given values of $M$ and $J$. Observe also that for fixed total $(M, J)$ we can vary, say, the mass and spin of the black ring, while adjusting the mass and spin of the central black hole to add up to the total $M$ and $J$. These configurations, then, exhibit doubly-continuous nonuniqueness.

We can similarly consider multiple-ring solutions. Di-rings, with two concentric black rings rotating on the same plane, were first constructed in [150]; [85] re-derived them using the BZ approach. Each new ring adds two parameters to the continuous degeneracy of solutions with given total $M$ and $J$.

Note that the surface gravities (i.e., temperatures) and angular velocities of disconnected components of the horizon are in general different. Equality of these 'intensive parameters' is a necessary condition for thermal equilibrium - and presumably also for mergers in phase space to solutions with connected horizon components; see Section 6.2. So these multiple-black-hole configurations cannot, in general, exist in thermal equilibrium (this is besides the problems of constructing a Hartle-Hawking state when ergoregions are present [157]). The curves for solutions, where all 
disconnected components of the horizon have the same surface gravities and angular velocities, are presented in Figure 10 (see [73]). All continuous degeneracies are removed, and black Saturns are always subdominant in total horizon area. It is expected that no multiple-ring solutions exist in this class.

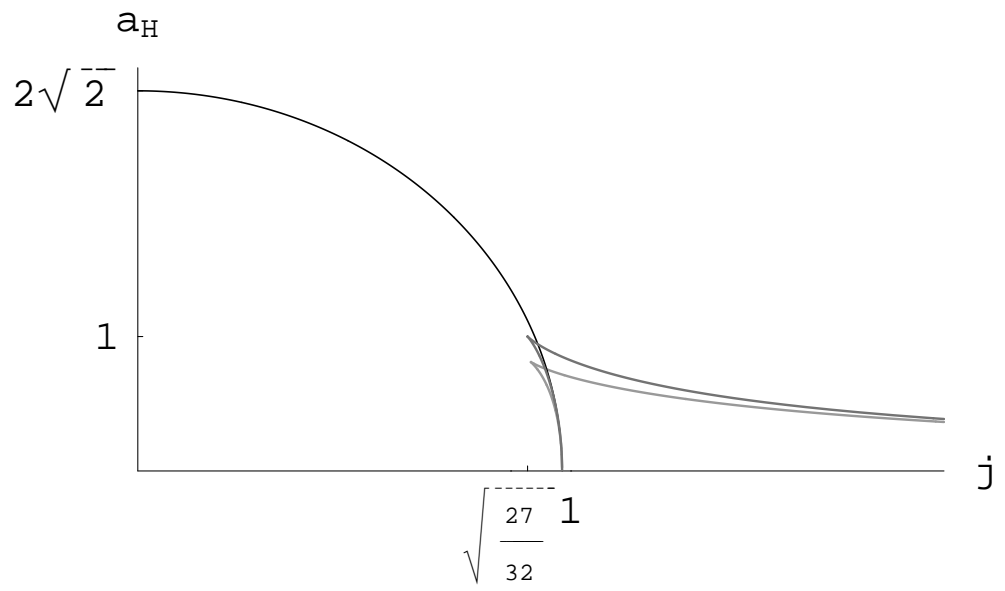

Figure 10: Curves $a_{H}(j)$ for phases of five-dimensional black holes with a single angular momentum: MP black hole (black), black ring (dark gray), black Saturn (light gray). We only include those black Saturns, where the central black hole and the black ring have equal surface gravities and angular velocities. The three curves meet tangentially at a naked singularity at $j=1, a_{H}=0$. The cusp of the black-ring curve occurs at $j=\sqrt{27 / 32} \approx 0.9186, a_{H}=1$. The cusp of the black-Saturn curve is at $j \approx 0.9245$, with area $a_{H} \approx 0.81$.

It is also possible to have two black rings lying and rotating on orthogonal, independent planes. Such bicycling black rings have been constructed using the BZ method [152, 78], and provide a way of obtaining configurations with arbitrarily large values of both angular momenta for fixed mass - which cannot be achieved simultaneously for both spins, either by the MP black holes or by doubly-spinning black rings. The solutions in $[152,78]$ are obtained by applying to each of the two rings the kind of transformations that generate the singly-spinning black ring. Thus each black ring possesses angular momentum only on its plane, along the $S^{1}$, but not in the orthogonal plane, on the $S^{2}$ - nevertheless, they drag each other so that the two horizon angular velocities are both nonzero on each of the two horizons. The solutions contain four free parameters, corresponding to, e.g., the masses of each of the rings and their two angular momenta. It is clear that a more general, six-parameter solution must exist in which each black ring has both angular momenta turned on.

It can be argued, extending the arguments in [73], that multiple-black-hole solutions allow one to cover the entire $\left(j_{1}, j_{2}\right)$ phase plane of five-dimensional solutions. It would be interesting to determine for which parameter values these multiple black holes have the same surface gravity and angular velocities on all disconnected components of the horizon. With this constraint, multiple-black-hole solutions still allow one to cover a larger region of the $\left(j_{1}, j_{2}\right)$ plane than already covered by solutions with a connected horizon;see Figure 7. For instance, it can be argued that some bicycling black rings (within the six-parameter family mentioned above for which the four angular velocities of the entire system can be varied independently) should satisfy these 'thermal equilibrium' conditions; as we have seen, a doubly-spinning black ring can have $\Omega_{1}=\Omega_{2}$. Thus, if we consider two identical doubly-spinning thin black rings, one on each of the two planes, then we can make them have $S^{1}$-angular velocity equal to the $S^{2}$-angular velocity of the other ring in the orthogonal plane. These solutions then lie along the lines $\left|j_{1}\right|=\left|j_{2}\right|$, reaching arbitrarily large

Living Reviews in Relativity

http: //www . livingreviews . org/lrr-2008-6 
$\left|j_{1,2}\right|$, which is not covered by the single-black-hole phases in Figure 7. Clearly, there will also exist configurations extending continuously away from this line.

Black Saturns with a single black ring that satisfy 'thermal equilibrium' conditions should also exist. In fact, the possibility of varying the temperature of the ring by tuning the rotation in the $S^{2}$ might allow one to cover portions of the $\left(j_{1}, j_{2}\right)$ plane beyond Figure 7 . If so, this would be unlike the situation with a single rotation, where thermal-equilibrium Saturns lie within the range of $j$ of black rings, Figure 10 .

The Weyl ansatz of Section 5.2.1 enables one to easily generate solutions in $d=5$ with multiple black holes of horizon topology $S^{3}$, which are asymptotically flat [82, 231]. However, all these solutions possess conical singularities reflecting the attraction between the different black holes. It seems unlikely that the extension to include off-diagonal metric components (rotation and twists) could eliminate these singularities and yield balanced solutions.

\subsection{Stability}

The linearized perturbations of the black-ring metric (50) have not yielded to analytical study. The apparent absence of a Killing tensor prevents the separation of variables even for massless scalar-field perturbations. In addition, the problem of decoupling the equations to find a master equation for linearized gravitational perturbations, already present for the Myers-Perry solutions, is, if anything, exacerbated for black rings.

Studies of the classical stability of black rings have, therefore, been mostly heuristic. Already the original paper [83] pointed out that very thin black rings locally look like boosted black strings (this was made precise in [71]), which were expected to suffer from GL-type instabilities. The instability of boosted black strings was indeed confirmed in [144]. Thus, thin black rings are expected to be unstable to the formation of ripples along their $S^{1}$ direction. This issue was examined in further detail in [76], which found that thin black rings seem to be able to accommodate unstable GL modes down to values $j \sim O(1)$. Thus, it is conceivable that a large fraction of black rings in the thin branch, and possibly all of this branch, suffer from this instability. The ripples rotate with the black ring and then should emit gravitational radiation. However, the timescale for this emission is much longer than the timescale of the fastest GL mode, so the pinchdown created by this instability will dominate the evolution, at least initially. The final fate of this instability of black rings depends on the endpoint of the GL instability, but it is conceivable, and compatible with an increment of the total area, that the black-ring fragments separate into smaller black holes that then fly away.

Another kind of instability was discussed in [76]. By considering off-shell deformations of the black ring (namely, allowing for conical singularities), it is possible to compute an effective potential for radial deformations of the black ring. Fat black rings sit at maxima of this potential, while thin black rings sit at minima. Thus, fat black rings are expected to be unstable to variations of their radius, and presumably collapse to form MP black holes. The analysis in [76] is in fact consistent with a previous, more abstract analysis of local stability in [2]. This is based on the 'turning-point' method of Poincaré, which studies equilibrium curves for phases near bifurcation points. For the case of black rings, one focuses on the cusp, where the two branches meet. One then assumes that these curves correspond to extrema of some potential, e.g., an entropy, that can be defined everywhere on the plane $\left(j, a_{H}\right)$. The cusp then corresponds to an inflection point of this potential at which a branch of maxima and a branch of minima meet. By continuity, the branch with the higher entropy will be the most stable branch, and the one with lower entropy will be unstable. Thus, for black rings an unstable mode is added when going from the upper (thin) to the lower (fat) branch. This is precisely as found in [76] from the mechanical potential for radial deformations.

Thus, a large fraction of all single-spin neutral black rings are expected to be classically unstable, 
and it remains an open problem whether a window of stability exists for thin black rings with $j \sim O(1)$. The stability, however, can improve greatly with the addition of charges and dipoles.

Doubly-spinning black rings are expected to suffer from similar instabilities. Insofar as a fat ring branch that meets at a cusp with a thin ring branch exists, the fat rings are expected to be unstable. Very thin rings are also expected to be unstable to GL-perturbations that form ripples. The angular momentum on the $S^{2}$ may be redistributed nonuniformly along the ring, with the larger blobs concentrating more spin. In addition, although it has been suggested that superradiant ergoregion instabilities associated to rotation of the $S^{2}$ might exist [68], a proper account of the asymptotic behavior of super-radiant modes needs to be made before concluding that the instability is actually present.

Much of what we can say about the classical stability of black Saturns and multiple rings follows from what we have said above for each of its components, e.g., if their rings are thin enough, they are expected to be GL-unstable. We know essentially nothing about what happens when the gravitational interaction among the black objects involved is strong. For instance, we do not know if the GL instability is still present when a thin black ring lassoes at very close range a much larger, central, MP black hole.

Massive geodesics on the plane of a black ring (see [143]) show that a particle at the center of the $S^{1}$ is unstable to migrating away towards the black ring. This suggests that a black Saturn with a small black hole at the center of a larger black ring should be unstable. One possibility for a different instability of black Saturns appears from the analysis of counter-rotating configurations in [77]. For large enough counter-rotation, the Komar-mass of the central black hole vanishes and then becomes negative. By itself, this does not imply any pathology, as long as the total ADM mass is positive and the horizon remains regular, which it does. However, it suggests that the counter-rotation in this regime becomes so extreme that the black hole might tend to be expelled off the plane of rotation.

Clearly, the classical stability of all, old and new, rotating black-hole solutions of five-dimensional general relativity remains largely an open problem, where much work remains to be done. 


\section{Vacuum Solutions in More Than Five Dimensions}

With no available techniques to construct asymptotically-flat exact solutions beyond those found by Myers and Perry, the situation in $d \geq 6$ is much less developed than in $d=5$. Recall that the symmetry requirements for the class of solutions (66) are incompatible with the asymptotic symmetries of globally flat space in $d>5$; metrics of type (66) necessarily involve directions with nonflat asymptotics (e.g., infinitely-extended black strings and branes) and/or asymptotic compact Kaluza-Klein circles. In order to preserve asymptotic flatness one would instead ask for rotational symmetry around 'axes' that are hyperplanes of a codimension larger than two, but the integrability of the resulting equations [34, 35] remains essentially an unsolved problem.

However, despite the paucity of exact solutions, there are strong indications that the variety of black holes that populate general relativity in $d \geq 6$ is vastly larger than in $d=4,5$. A first indication came from the conjecture in [81] of the existence of black holes with spherical horizon topology but with axially-symmetric 'ripples' (or 'pinches'). The plausible existence of black rings in any $d \geq 5$ was argued in [144, 76]. More recently, [80] has constructed approximate solutions for black rings in any $d \geq 5$ and then exploited the conjecture of [81] to try to draw a phase diagram with connections and mergers between the different expected phases. In the following we summarize these results.

\subsection{Approximate solutions from curved thin branes}

In the absence of exact techniques, [80] resorts to approximate constructions, in particular to the method of matched asymptotic expansions previously used in the context of black holes localized in Kaluza-Klein circles in $[126,115,156,116]^{7}$. The basic idea is to find two widely separated scales in the problem, call them $R_{1}$ and $R_{2}$, with $R_{2} \ll R_{1}$, and then try to solve the equations in two limits; first, as a perturbative expansion for small $R_{2}$, and then in an expansion in $1 / R_{1}$. The former solves the equations in the far region $r \gg R_{2}$ in which the boundary condition, e.g., asymptotic flatness, fixes the integration constants. The second expansion is valid in the near region $r \ll R_{1}$. In order to fix the integration constants in this case, one matches the two expansions in the overlap region $R_{2} \ll r \ll R_{1}$ in which both approximations are valid. The process can then be iterated to higher orders in the expansion; see [115] for an explanation of the systematics involved.

In order to construct a black ring with horizon topology $S^{1} \times S^{d-3}$, we take the scales $R_{1}, R_{2}$ to be the radii of the $S^{1}$ and $S^{d-3}$, respectively ${ }^{8}$. To implement the above procedure, we take $R_{2}=r_{0}$, the horizon radius of the $S^{d-3}$ of a straight boosted black string, and $R_{1}=R$ the large circle radius of a very thin circular string. Thus, in effect, to first order in the expansion what one does is: (i) find the solution within the linearized approximation, i.e., for small $r_{0} / r$, around a Minkowski background for an infinitely-thin circular string with momentum along the circle; (ii) perturb a straight boosted black string so as to bend it into an arc of very large radius $1 / R$. Step (ii) not only requires matching to the previous solution in order to provide boundary conditions for the homogeneous differential equations; one also needs to check that the perturbations can be made compatible with regularity of the horizon.

It is worth noting that the form of the solution thus found exhibits a considerable increase in complexity when going from $d=5$, where an exact solution is available, to $d>5$; simple linear functions of $r$ in $d=5$ change to hypergeometric functions in $d>5$. We take this as an indication that exact closed analytical forms for these solutions may not exist in $d>5$.

We will not dwell here on the details of the perturbative construction of the solution (see [80] for this), but instead we shall emphasize that adopting the view that a black object is approximated

\footnotetext{
${ }^{7}$ The classical effective field theory of $[49,168]$ is an alternative to matched asymptotic expansions, which presumably should be useful as well in the context discussed in this section.

${ }^{8}$ The $S^{d-3}$ is not round for known solutions, but one can define an effective scale $R_{2}$ as the radius of a round $S^{d-3}$ with the same area.
} 
by a certain very thin black brane curved into a given shape can easily yield nontrivial information about new kinds of black holes. Eventually, of course, the assumption that the horizon remains regular after curving needs to be checked.

Consider then a stationary black brane, possibly with some momentum along its worldvolume, with horizon topology $\mathbb{R}^{p+1} \times S^{q}$, with $q=d-p-2$. When viewed at distances much larger than the size $r_{0}$ of the $S^{q}$, we can approximate the metric of the black-brane spacetime by the gravitational field created by an 'equivalent source' with distributional energy tensor $T_{\mu}^{\nu} \propto r_{0}^{q-1} \delta^{(q+1)}(r)$, with nonzero components only along directions tangent to the worldvolume, and where $r=0$ corresponds to the location of the brane. Now we want to put this same source on a curved, compact $p$-dimensional spatial surface in a given background spacetime (e.g., Minkowski, but possibly (anti-)de Sitter or others, too). In principle we can obtain the mass $M$ and angular momenta $J_{i}$ of the new object by integrating $T_{t}^{t}$ and $T_{t}^{i}$ over the entire spatial section of the brane worldvolume. Moreover, the total area $\mathcal{A}_{H}$ is similarly obtained by replacing the volume of $\mathbb{R}^{p}$ with the volume of the new surface. Thus, it appears that we can easily obtain the relation $\mathcal{A}_{H}\left(M, J_{i}\right)$ in this manner.

There is, however, the problem that having changed the embedding geometry of the brane, it is not guaranteed that the brane will remain stationary. Moreover, $\mathcal{A}_{H}$ will be a function not only of $\left(M, J_{i}\right)$, but will also depend explicitly on the geometrical parameters of the surface. However, we would expect that in a situation of equilibrium, some of these geometrical parameters would be fixed dynamically by the mechanical parameters $\left(M, J_{i}\right)$ of the brane. For instance, take a boosted string and curve it into a circular ring so that the linear velocity turns into angular rotation. If we fix the mass and the radius, then the ring will not be in equilibrium for every value of the boost, i.e., of the angular momentum; so, there must exist a fixed relation $R=f(M, J)$. This is reflected in the fact that, in the new situation, the stress-energy tensor is in general not conserved, $\nabla_{\mu} T^{\mu \nu} \neq 0$; additional stresses would be required to keep the brane in place. An efficient way of imposing the brane equations of motion is, in fact, to demand conservation of the stress-energy tensor. In the absence of external forces, the classical equations of motion of the brane derived in this way are [28]

$$
K_{\mu \nu}^{\rho} T^{\mu \nu}=0,
$$

where $K^{\rho}{ }_{\mu \nu}$ is the second fundamental tensor, characterizing the extrinsic curvature of the embedding surface spanned by the brane worldvolume. For a string on a circle of radius $R$ in flat space, parameterized by a coordinate $z \sim z+2 \pi R$, this equation becomes

$$
\frac{T_{z z}}{R}=0
$$

In $d=5$, this can be seen to correspond to the condition of the absence of conical singularities in solution (50), in the limit of a very thin black ring [71]. [80] shows that this condition is also required in $d \geq 6$ in order to avoid curvature singularities on the plane of the ring.

In general, Equation (110) constrains the allowed values of the parameters of a black brane that can be put on a given surface. [80] easily derives, for any $d \geq 5$, that the radius $R$ of thin rotating black rings of given $M$ and $J$ is fixed to

$$
R=\frac{d-2}{\sqrt{d-3}} \frac{J}{M}
$$

so large $R$ corresponds to large spin for fixed mass. The horizon area of these thin black rings goes like

$$
\mathcal{A}_{H}(M, J) \propto J^{-\frac{1}{d-4}} M^{\frac{d-2}{d-4}} .
$$

This is to be compared to the value for ultraspinning MP black holes in $d \geq 6$ (cf. Equations (40), $(41)$ as $\nu \rightarrow 0)$,

$$
\mathcal{A}_{H}(M, J) \propto J^{-\frac{2}{d-5}} M^{\frac{d-2}{d-5}}
$$


This shows that in the ultraspinning regime the rotating black ring has larger area than the MP black hole.

\subsection{Phase diagram}

Using the dimensionless area and spin variables (21), Equation (113) allows one to compute the asymptotic form of the curve $a_{H}(j)$ in the phase diagram at large $j$ for black rings. However, when $j$ is of order one, the approximations in the matched asymptotic expansion break down, and the gravitational interaction of the ring with itself becomes important. At present we have no analytical tools to deal with this regime for generic solutions. In most cases, numerical analysis may be needed to obtain precise information.

Nevertheless, [80] contains advanced heuristic arguments, which propose a completion of the curves that is qualitatively consistent with all the information available at present. A basic ingredient is the observation in [81], discussed in Section 4.1, that in the ultraspinning regime in $d \geq 6$, MP black holes approach the geometry of a black membrane $\approx \mathbb{R}^{2} \times S^{d-4}$ spread out along the plane of rotation.

We have already discussed how using this analogy, [81] argues that ultraspinning MP black holes should exhibit a Gregory-Laflamme-type of instability. Since the threshold mode of the GL instability gives rise to a new branch of static nonuniform black strings and branes [117, 120, 248], [81] argues that it is natural to conjecture the existence of new branches of axisymmetric 'lumpy' (or 'pinched') black holes, branching off from the MP solutions along the stationary axisymmetric zero-mode perturbation of the GL-like instability.

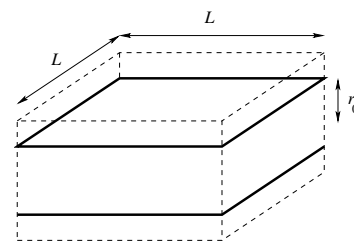

(i)
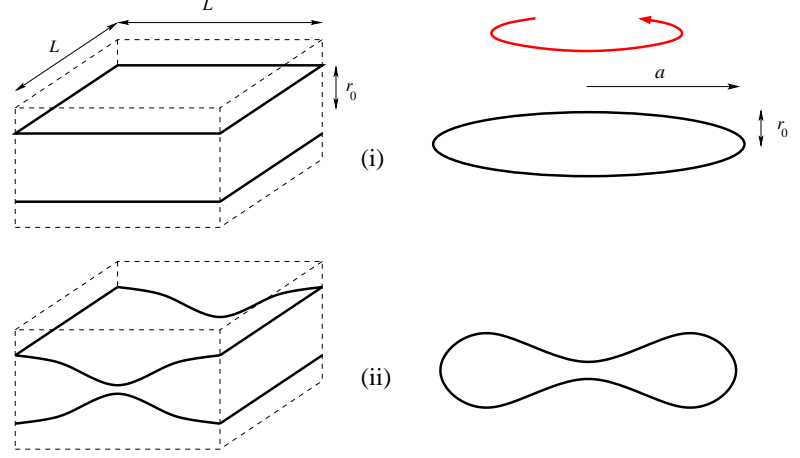

(ii)
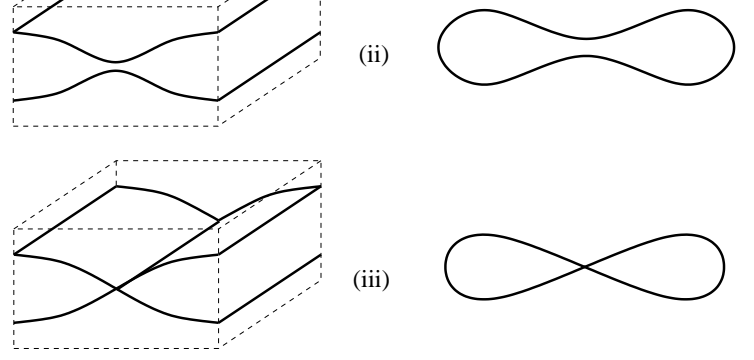

(iii)
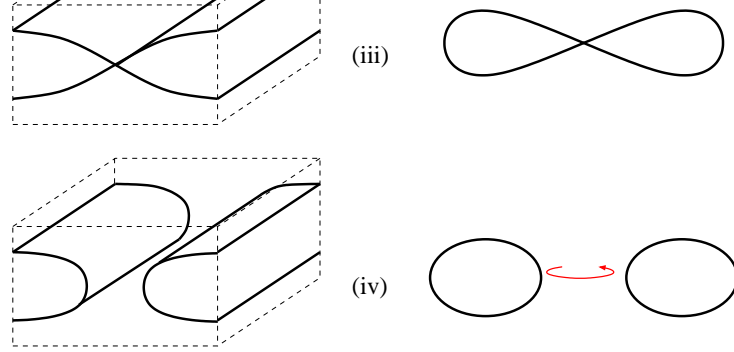

(iv)

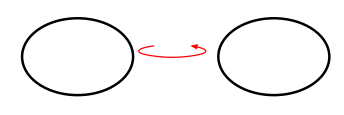

Figure 11: Correspondence between phases of black membranes wrapped on a two-torus of side $L$ (left) and quickly-rotating MP black holes with rotation parameter $a \sim L \geq r_{0}$ (right: must be rotated along a vertical axis): (i) uniform black membrane and MP black hole; (ii) nonuniform black membrane and pinched black hole; (iii) pinched-off membrane and black hole; (iv) localized black string and black ring (reproduced from [80]). 
[80] develops further this analogy, and draws a correspondence between the phases of black membranes and the phases of higher-dimensional black holes, illustrated in Figure 11. Although the analogy has several limitations, it allows one to propose a phase diagram in $d \geq 6$ of the form depicted in Figure 12, which should be compared to the much simpler diagram in five dimensions, Figure 10. Observe the presence of an infinite number of black holes with spherical topology, connected via merger transitions to MP black holes, black rings, and black Saturns. Of all multipleblack-hole configurations, the diagram only includes those phases in which all components of the horizon have the same surface gravity and angular velocity; presumably, these are the only ones that can merge to a phase with connected horizon. Even within this class of solutions, the diagram is not expected to contain all possible phases with a single angular momentum; blackfolds with other topologies must likely be included as well. The extension to phases with several angular momenta also remains to be done.

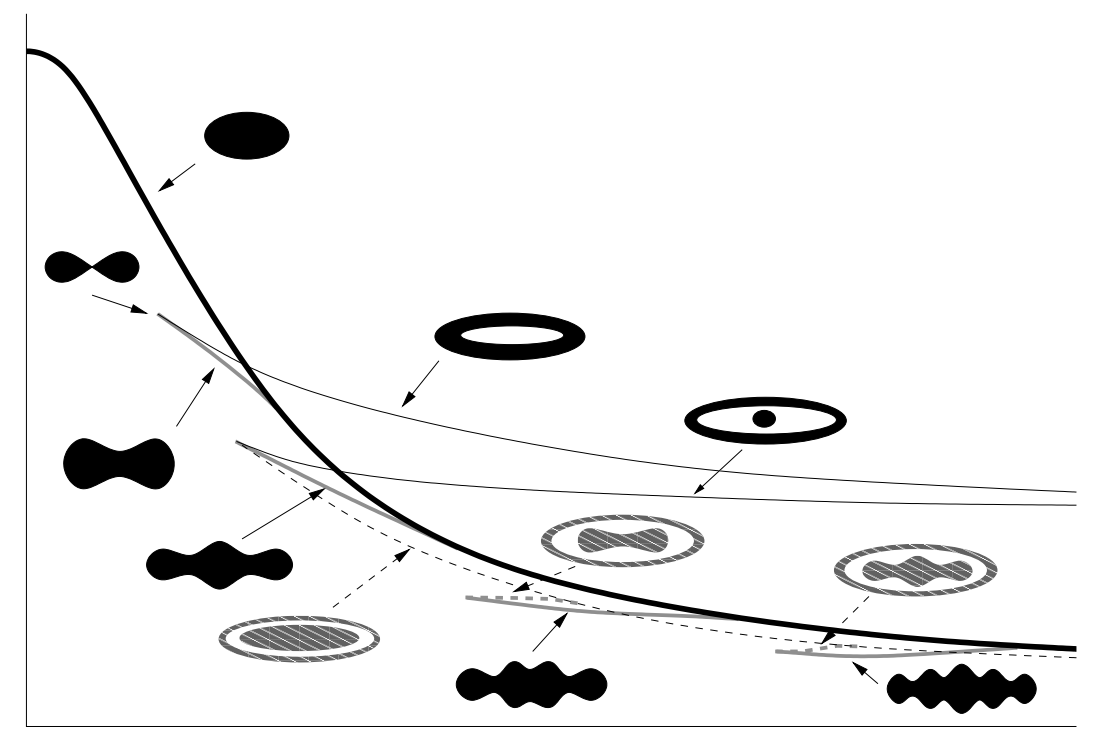

Figure 12: Proposal of [80] for the phase curves $a_{H}(j)$ of thermal equilibrium phases in $d \geq 6$. The solid lines and figures have significant arguments in their favor, while the dashed lines and figures might not exist and admit conceivable, but more complicated, alternatives. Some features have been drawn arbitrarily; at any given bifurcation, and in any dimension, smooth connections are possible instead of swallowtails with cusps; also, the bifurcation into two black-Saturn phases may happen before, after, or right at the merger with the pinched black hole. Mergers to di-rings or multiple-ring configurations that extend to asymptotically large $j$ seem unlikely. If thermal equilibrium is not imposed, the whole semi-infinite strip $0<a_{H}<a_{H}(j=0), 0 \leq j<\infty$ is covered, and multiple rings are possible.

Indirect evidence for the existence of black holes with pinched horizons is provided by the results of [180], which finds 'pinched plasma-ball' solutions of fluid dynamics that are CFT duals of pinched black holes in six-dimensional AdS space. The approximations involved in the construction require that the horizon size of the dual black holes be larger than the AdS curvature radius, and thus do not admit a limit to flat space. Nevertheless, their existence provides an example, if indirect, that pinched horizons make an appearance in $d=6$ (and not in $d=5$ ). 


\subsection{Stability}

The situation in $d \geq 6$ is very similar to what we described for $d=5$ in Section 5.4 ; most of what we know is deduced by heuristic analogies and approximate methods. The following prototypic instabilities can be easily identified:

- GL-type instabilities in ultraspinning regimes. Both MP black holes and black rings approach, in this regime, string or membrane-like configurations that are expected to be unstable to growing nonuniformities along the 'long' directions on the horizon. Such instabilities give rise, in the cases where the nonuniformities can remain stationary, to zero modes that branch off into new solutions with broken symmetry.

- Turning-point instabilities. Given the phase diagram with different curves $a_{H}(j)$ in it, we expect that near a point where different phases meet, the branch with lesser area should have one more unstable mode than those with greater area. This happens along black-ring curves at the turning points where $j$ reaches a minimum. The instability is presumably related to radial deformations of the ring. It also happens at mergers between phases.

We end this section emphasizing that, presumably, new concepts and tools are required for the characterization of black holes in $d \geq 6$, let alone for their explicit construction.

The general problem of the dynamical linearized stability of MP black holes, in particular in the case with a single rotation, becomes especially acute for the determination of possible black-hole phases in $d \geq 6$. The arguments in favor of an ultraspinning instability seem difficult to evade, so a most pressing problem is to locate the point (i.e., the value of $j$ ) at which this instability appears as a stationary mode, and then perturb the solution along this mode to determine the direction in which the new branch of solutions evolves. 


\section{Solutions with a Gauge Field}

\subsection{Introduction}

Black holes with nontrivial gauge fields play an important role in string theory, not least because the types of charge that they carry help identify their microscopic constituents (e.g., branes). In this section we shall review briefly such solutions for $d \geq 5$. We shall concentrate exclusively on solutions of maximal supergravity theories arising as consistent toroidal reductions of $d=10,11$ supergravity.

The bosonic sector of maximal supergravity theories contains massless scalars taking values in some coset space $G / H$ where $G$ is noncompact and $H$ is compact. Since the scalars are massless, their asymptotic values (for an asymptotically-flat solution) are arbitrary, i.e., they are moduli. $G$ is a global symmetry of the supergravity theory (broken to a discrete U-duality symmetry in string/M-theory). By acting with $G$ we can choose the moduli to be anything convenient. Given a particular choice for the moduli, the global symmetry group is broken to H. See, e.g., [210] for a review of this.

The only type of conserved gauge charge that an asymptotically flat solution can carry is electric charge with respect to a 2 -form field strength (or magnetic charge with respect to a $d-2$ form field strength, but this can always be dualized into a 2-form). The group $H$ acts nontrivially on the charges of a solution. Hence, the strategy in looking for black-hole solutions is to identify a "seed" solution with a small number of parameters, from which a solution with the most general charge assignments can be obtained by acting with $H$. For example, dimensional reduction of $d=11$ supergravity on $T^{6}$ gives the maximal $\mathcal{N}=4, d=5$ supergravity, which has 27 Abelian vectors and 42 scalars parameterizing the coset $E_{6(6)} / U S p(8)$. It turns out that the a solution with 27 independent charges can be obtained by acting with $U S p(8)$ on a seed solution with just 3 charges [58].

\subsection{Topologically-spherical black holes}

\subsubsection{Nonextremal solutions}

The construction of stationary, charged, topologically-spherical, black-hole solutions ("charged Myers-Perry") in maximal supergravity theories was described in [58]. It turns out that the seed solutions are the same as for toroidal compactifications of heterotic string theory. The latter seed solutions were constructed in [63] for $d=5$ and $[64,184]$ for $6 \leq d \leq 9$. In addition to their mass and angular momenta, they are parameterized by three electric charges in $d=5$ and two electric charges for $6 \leq d \leq 9$. For $d=10$ (type IIA theory), the solution describing a rotating black hole charged with respect to the Ramond-Ramond 2-form field strength (i.e., D0-brane charge) can be obtained from the general rotating brane solution given in [55].

In the limit of vanishing angular momenta, all of these solutions reduce to $d>4$ generalizations of the Reissner-Nordström solution. This static case is the only case for which linearized stability has been investigated. The spherical symmetry of static solutions permits a scalar/vector/tensor decomposition of perturbations. For Reissner-Nordström solutions of $d>4$ Einstein-Maxwell theory, decoupled equations governing each type have been obtained [164]. These have been used to prove analytically the stability of tensor and vector perturbations for $d \geq 5$ and scalar perturbations for $d=5$ [164]. Numerical studies have revealed that scalar perturbations are also stable for $6 \leq d \leq 11$ [169].

Living Reviews in Relativity

http://www . livingreviews . org/lrr-2008-6 


\subsubsection{Supersymmetric solutions: the Breckenridge-Myers-Peet-Vafa (BMPV) black hole}

Black holes saturating a Bogomolnyi-Prasad-Sommerfield (BPS) inequality play an important role in string theory. The canonical example is the first black-hole entropy calculation [229], in which the BPS condition provided the justification for relating the Bekenstein-Hawking entropy of a classical black-hole solution to the statistical entropy of a microscopic brane configuration.

The only known asymptotically-flat BPS black-hole solutions occur for $d=4,5$. For $d=5$, one can obtain BPS black-hole solutions as a limit of the nonextremal charged rotating solutions just discussed. Starting from the six-parameter seed solution of [63] one can obtain a four-parameter BPS black hole: the BMPV black hole [17, 242]. The solution has equal angular momenta $J_{1}=$ $J_{2}=J$ and the four parameters are $J$ and the three electric charges. The mass is fixed by the BPS relation. Note that one loses two parameters in the BPS limit; this is because the BPS limit and the extremal limit are distinct for rotating black holes - a BPS black hole is necessarily extremal, but the converse is untrue. As for the Myers-Perry solution, the equality of the angular momenta gives rise to a non-Abelian isometry group $\mathbb{R} \times U(1) \times S U(2)$. Classical properties of the BMPV solution have been discussed in detail in $[102,106]$.

\subsection{Black rings with gauge fields}

\subsubsection{Dipole rings}

Black holes can only carry electric charge with respect to a two-form. However, higher-rank $p$-forms may also be excited by a black hole, even though there is no net charge associated with them. This occurs naturally for black rings. Consider a black string in $d$ dimensions. A string carries electric charge associated with a three-form field strength $H$. The electric charge is proportional to the integral of $\star H$ over a $(d-3)$-sphere that links the string. Now consider a black ring with topology $S^{1} \times S^{d-3}$ formed by bending the string into a loop and giving it angular momentum around the $S^{1}$. This would be asymptotically flat and, hence, the charge associated with $H$ would be zero. Nevertheless, $H$ would be nonzero. Its strength can be measured by the flux of $\star H$ through a $S^{d-3}$ linking the ring, which is no longer a conserved quantity but rather a nonconserved electric dipole. Such a ring would have three parameters, but would only have two conserved charges (mass and angular momentum), hence it would exhibit continuous nonuniqueness.

Exact solutions describing such "dipole rings" have been constructed in $d=5$ [79]. In $d=5$, one can dualize a 3 -form $H$ to a two-form, so these solutions carry magnetic dipoles with respect to two-form field strengths. The dipole rings of [79] are solutions of $\mathcal{N}=1, d=5$ supergravity coupled to two vector multiplets, which is a theory with $U(1)^{3}$ gauge group that can be obtained by consistent truncation of the maximal $d=5$ supergravity theory. These rings are characterized by their mass $M$, angular momentum $J_{1}$ around the $S^{1}$ of the horizon, and three dipoles $q_{i}$. The angular momentum $J_{2}$ on the $S^{2}$ of the horizon vanishes. They have the same $\mathbb{R} \times U(1)^{2}$ symmetry as vacuum black rings. These solutions are seed solutions for the construction of solutions of maximal $d=5$ supergravity with 27 independent dipoles obtained by acting with $U S p(8)$ as described above. One would expect the existence of more general dipole-ring solutions with two independent nonzero angular momenta, but these are yet to be discovered.

\subsubsection{Charged black rings, supersymmetric black rings}

Black rings can also carry conserved electric charges with respect to a 2-form field strength (the first regular example was found in [70], which can be regarded as having two charges and one dipole in a $U(1)^{3}$ supergravity theory; see also [71]). Hence there is the possibility that they can saturate a BPS inequality. The first example of a supersymmetric black-ring solution was obtained 
for minimal $d=5$ supergravity in [74] using a canonical form for supersymmetric solutions of this theory [101]. This was then generalized to a supersymmetric black-ring solution of the $U(1)^{3}$ supergravity in $[12,75,100]$. The latter solution has 7 independent parameters, which can be taken to be the 3 charges, 3 dipoles and $J_{1}$. The mass is fixed by the BPS relation and $J_{2}$ is determined by the charges and dipoles. See [84] for more detailed discussion of these solutions.

The most general, stationary, black-ring solution of the $U(1)^{3}$ supergravity theory is expected to have nine parameters, since one would expect the three charges, three dipoles, two angular momenta and the mass to be independent. This solution has not yet been constructed. Note that the general non-BPS solution should have two more parameters than the general BPS solution, just as for topologically-spherical rotating black holes. The most general, known, nonextremal solution [72] has seven parameters, and was obtained by applying solution-generating transformations to the dipole ring solutions of [79]. This solution does not have a regular BPS limit, and there is no limit in which it reduces to a vacuum black ring with two angular momenta.

It has been argued that a nine parameter black-ring solution could not be a seed solution for the most general black-ring solution of maximal $d=5$ supergravity [181]. By acting with $U S p(8)$, one can construct a solution with 27 independent charges from a seed with 3 independent charges or one can construct a solution with 27 independent dipoles from a seed with three independent dipoles. However, one cannot do both at once. If one wants to construct a solution with 27 independent charges and 27 independent dipoles from a seed solution with three independent charges, then this seed must have 15 independent dipoles, and hence (including the mass and angular momenta) 21 parameters in total. The seed solution for the most general supersymmetric black ring in maximal $d=5$ supergravity is expected to have 19 parameters [181].

\subsection{Solution-generating techniques}

[16] develops solution-generating techniques in minimal $d=5$ supergravity, based on U-duality properties of the latter. By applying one such transformation to the neutral doubly-spinning black ring of Section 5.1.2, they obtain a new charged ring solution of five-dimensional supergravity. However, this solution suffers from the same problem of Dirac-Misner singularities that [71] described when a neutral single-spin ring seed is used. It appears that the problem could be solved, like in [72], by including an additional parameter that is then tuned to cancel the pathologies. It also seems possible that, like in [71], the neutral doubly-spinning black ring is a good seed for black rings with two charges, one dipole, and two independent angular momenta in the $U(1)^{3}$ supergravity theory.

Besides the solution-generating techniques based on string theory and supergravity (sequences of boosts and dualities) there have been a number of analyses of the Einstein-Maxwell(-dilaton) equations leading to other techniques for generating stationary solutions. [146] studies general properties of the Einstein-Maxwell equations in $d$-dimensions with $d-3$ commuting Killing-vector fields. [233] shows how four-dimensional, vacuum, stationary, axisymmetric solutions can be used to obtain static, axisymmetric solutions of five-dimensional dilaton gravity coupled to a two-form gauge field. Also, within the class of five-dimensional stationary solutions of the Einstein-Maxwell(dilaton) equations with two rotational symmetries, solution-generating techniques have been developed in [175] and [251, 253]. Note that many of these papers do not take into account the Chern-Simons term present in $d=5$ supergravity. This term is relevant when both electric and magnetic components of the gauge field are present. Thus, it can be ignored for electrically-charged static solutions, which do not give rise to magnetic dipoles. It does not play a role, either, for the dipole rings of [79], which has allowed a systematic derivation of these solutions [252, 254].

Living Reviews in Relativity

http: //www . livingreviews . org/lrr-2008-6 


\subsection{Multiple-black-hole solutions}

The inclusion of electric charge makes it considerably easier to construct multiple-black-hole solutions than in the vacuum case discussed above. In $d=4$, there exist well-known static solutions describing multiple extremal Reissner-Nordström black holes held in equilibrium by a cancellation of electric repulsion and gravitational attraction [131]. Similar static solutions can be constructed in $d>4$ [198]. However, although the $d=4$ solutions have smooth horizons [131], the $d>4$ solutions have horizons of low differentiability [246, 22].

In $d=4,5$, multiple-black-hole solutions can be supersymmetric. Supersymmetry makes it easy to write down stationary solutions corresponding to multiple BMPV black holes [102]. However, the regularity of these solutions has not been investigated. Presumably they are no smoother than the static solutions just mentioned. Although electromagnetic and gravitational forces cancel, one might expect spin-spin interactions to play a role in these solutions, perhaps leading to even lower smoothness, unless the spins are aligned. Note that, in general, superposition of these black holes breaks all symmetries of a single central BMPV solution except for time-translation invariance.

Supersymmetric solutions describing stationary superpositions of multiple concentric black rings have also been constructed [99, 100]. The rings have a common center, and can either lie in the same plane, or in orthogonal planes. The superposition does not break any symmetries. This may be the reason that these solutions have smooth horizons.

Turning to nonextremal solutions, one would certainly expect generalizations of the solutions of section 5.3 with nontrivial gauge fields. A solution describing a Myers-Perry black hole with a concentric dipole ring is presented in [255]. 


\section{General Results and Open Problems}

\subsection{Introduction}

In four dimensions, the black-hole uniqueness theorem states that there is at most one stationary, asymptotically-flat, vacuum black-hole solution with given mass and angular momentum: the Kerr black hole. The coexistence of Myers-Perry black holes and black rings shows explicitly that blackhole uniqueness is violated in five dimensions. By now there is strong evidence that this is even more dramatically true in more than five dimensions. However, even if higher-dimensional black holes are not uniquely characterized by their conserved charges, we can still hope to classify them. A major goal of research in higher-dimensional general relativity is to solve the classification problem: determine all stationary, asymptotically-flat black-hole solutions of the higher-dimensional vacuum Einstein equation (or Einstein equation coupled to appropriate matter). We are still a long way from this goal, but partial progress has been made, as we shall review below.

\subsection{Black-hole topology}

Logically, the first step in the proof of the $d=4$ black-hole uniqueness theorem is Hawking's blackhole topology theorem [132], which states that (a spatial cross-section of) the event horizon must be topologically $S^{2}$. The strongest version of this theorem makes use of topological censorship. Loosely, this is the statement that, in an asymptotically-flat and globally-hyperbolic spacetime obeying the null-energy condition, every causal curve beginning and ending at infinity can be homotopically deformed to infinity. This can be used to prove the topology theorem for stationary [48], and even nonstationary [153, 95], black holes.

The existence of black rings demonstrates that topologically nonspherical horizons are possible for $d>4$. But there are still restrictions on the topology of the event horizon. It has been shown $[98,96]$ that a stationary, asymptotically-flat, black-hole spacetime, obeying the dominant energy condition, must have a horizon that is "positive Yamabe", i.e., it must admit a metric of positive Ricci scalar. This restricts the allowed topologies. After $d=4$, the strongest restriction is for $d=5$, in which case the topology must be a connected sum of spherical spaces (3-spheres with identifications) and $S^{1} \times S^{2}$ 's.

Another topological restriction arises from cobordism theory. Consider surrounding the black hole with a large sphere. Let $\Sigma$ denote a spacelike hypersurface that runs from this sphere down to the event horizon, which it intersects in some compact surface $H$. Then $\Sigma$ is a cobordism between $H$ and a sphere. The existence of such a cobordism imposes topological restrictions on $H$. These have been discussed in [136]. The results are much less restrictive than those just mentioned.

It may be that horizon topology is not very useful for classifying black holes in $d \geq 6$. As we discussed in the introduction 1 , it is the combination of extended horizons with the novel possibilities for rotation that give higher-dimensional black holes much of their richness. For a black ring with horizon topology $S^{1} \times S^{2}$, the two factors differ in that the $S^{1}$, being contractible, needs rotation to be stabilized, whereas the $S^{2}$ is already a minimal surface. In $d \geq 6$ we can envisage even more complicated situations arising from the bending into different surfaces of the worldvolume directions of a variety of black p-branes. Not only horizon topology, but also, and more importantly, extrinsic geometry and dynamical considerations seem to be relevant to the existence of these black holes.

\subsection{Uniqueness of static black holes}

In $d=4$ dimensions, the Schwarzschild solution is the unique, static, asymptotically-flat, vacuum black-hole solution. The strongest version of this theorem allows for a possibly-disconnected event horizon, and the proof uses the positive energy theorem [19]. This proof can be extended to

Living Reviews in Relativity

http: //www. livingreviews .org/lrr-2008-6 
$d>4$-dimensions to establish uniqueness of the $d>4$-dimensional Schwarzschild solution amongst static vacuum solutions [110]. The method can also be generalized to prove a uniqueness theorem for static, asymptotically-flat, black-hole solutions of $d>4$-dimensional Einstein-Maxwell-dilaton theory; such black holes are uniquely characterized by their mass and charge and are described by generalized Reissner-Nordström solutions [109].

These theorems assume that there are no degenerate components of the horizon. This assumption can be eliminated for $d=4$ vacuum gravity [44, 46]. In Einstein-Maxwell theory, one can show that the only solutions with degenerate horizons are the Majumdar-Papapetrou multiReissner-Nordström solutions [47]. These results have been generalized to $d>4$ Einstein-Maxwell theory [218, 220].

In conclusion, the classification problem for static black holes has been solved, at least for the class of theories mentioned.

It must be noted, though, that the assumption of staticity is stronger than requiring vanishing total angular momentum. The existence of black Saturns (Section 5.3) shows that there exists an infinite number of solutions (with disconnected event horizons) characterized by a given mass and vanishing angular momentum.

\subsection{Stationary black holes}

In $d=4$, the uniqueness theorem for stationary black holes relies on Hawking's result that a stationary black hole must be axisymmetric [132]. This result has been generalized to higher dimensions [138]. More precisely, it can be shown that a stationary, nonextremal, asymptoticallyflat, rotating, black-hole solution of $d>4$ dimensional Einstein-Maxwell theory must admit a spacelike Killing vector field that generates rotations. Here "rotating" means that the Killing field that generates time translations is not null on the event horizon, i.e., the angular velocity is nonvanishing. However, it can be shown that a nonrotating black hole must be static for EinsteinMaxwell theory in $d=4$ [230] and $d>4$ [219], so this assumption can be eliminated. The result of [138] also applies in the presence of a cosmological constant (e.g., asymptotically anti-de Sitter black holes).

This theorem guarantees the existence of a single rotational symmetry. However, the known higher-dimensional black-hole solutions (i.e., Myers-Perry and black rings) admit multiple rotational symmetries; in $d$ dimensions there are $\lfloor(d-1) / 2\rfloor$ rotational Killing fields. Is there some underlying reason that this must be true, or is this simply a reflection of the fact that we are only able to find solutions with a lot of symmetry?

If there do exist solutions with less symmetry then they must be nonstatic (because of the uniqueness theorem for $d>4$ static black holes). One could look for evidence that such solutions exist by considering perturbations of known solutions [216]. For example, the existence of nonuniform black strings was first conjectured on the evidence that uniform strings exhibit a static zeromode that breaks translational symmetry. If a Myers-Perry black hole had a stationary zeromode that breaks some of its rotational symmetry then that would be evidence in favor of the existence of a new branch of solutions with less symmetry [216]. Alternatively, bifurcations could occur without breaking any rotational symmetry. As we have discussed, [80, 81] have conjectured that such bifurcations will happen in $d \geq 6$; see Figure 12. Either case could lead to nonuniqueness within solutions of spherical topology.

The formalism of [151] allows one to show that the only regular stationary perturbations of a Schwarzschild black hole lead into the MP family of black holes [162]. Thus, the MP solutions are the only stationary black holes that have a regular static limit.

The issue of how much symmetry a general stationary black hole must possess is probably the main impediment to progress with the classification problem. At present, the only uniqueness results for stationary higher-dimensional black holes assume the existence of multiple rotational 
symmetries. These results are for $d=5$; if one assumes the existence of two rotational symmetries then it can be shown that the Myers-Perry solution is the unique, stationary, nonextremal, asymptotically-flat, vacuum black-hole solution of spherical topology [194]. More generally, it has been shown that stationary, nonextremal, asymptotically-flat, vacuum black-hole solutions with two rotational symmetries are uniquely characterized by their mass, angular momenta, and rod structure (see Section 5.2.2.1) [139]. The remaining step in a full classification of all $d=5$ stationary vacuum black holes with two rotational symmetries is to prove that the only rod structures giving rise to regular black-hole solutions are those associated with the known (Myers-Perry and black ring) solutions.

The situation in $d \geq 6$ is much further away from a complete classification, even for the class of solutions with the maximal number of rotational symmetries. For instance, the tools to classify the (yet to be found) infinite number of families of solutions with 'pinched horizons' proposed in $[80,81]$ are still to be developed.

It is clear that the notion of black-hole uniqueness that holds in four dimensions, namely that conserved charges serve to fully specify a black hole, does not admit any simple extension to higher dimensions. This leaves us with two open questions: (a) what is the simplest and most convenient set of parameters that fully specify a black hole; (b) how many black-hole solutions with given conserved charges are relevant in a given physical situation.

Concerning the first question, we note that while the rod structure may provide the additional data to determine five-dimensional vacuum black holes, one may still desire a characterization in terms of physical parameters. In other words, since the dimensionless angular momenta $j_{a}$ are insufficient to specify the solutions, an adequate physical parameterization of the phase space of higher-dimensional black holes is still missing. [225, 241] have studied whether higher multipole moments may serve this purpose, but the results appear to be inconclusive.

The second question is more vague, as it hinges not only on the answer to the previous question, but also on the specification of the problem one is studying. It has been speculated that the conserved charges may still suffice to select a unique classical stationary configuration, if supplied with additional conditions, such as the specification of horizon topology or requiring classical stability [166]. In five dimensions we already know that horizon topology alone is not enough, since there are both thin and fat black rings with the same $j$. We have seen that, very likely, an even larger nonuniqueness occurs in all $d \geq 6$. Classical dynamical stability to linearized perturbations, which is not at all an issue in the four-dimensional classification, is presumably a much more restrictive condition, but even in five dimensions it is unclear whether it always picks out only one solution for a given $\left(j_{1}, j_{2}\right)$. One must also bear in mind that the classical instability of a black hole does not per se rule it out as a physically relevant solution; the time-scale of the instability must be compared to the time scale of the situation at hand. Some classical instabilities (e.g., ergoregion instabilities [25]) may be very slow.

It seems possible, although so far we are nowhere near having any compelling argument, that the requirement of classical, linearized stability, plus, possibly, horizon topology, suffices to fully specify a unique vacuum black-hole solution with given conserved charges. However, in the presence of gauge fields, this seems less likely, since dipoles not only introduce larger degeneracies but also tend to enhance the classical stability of the solutions.

\subsection{Supersymmetric black holes}

As mentioned earlier, the study of BPS black holes is of special importance in string theory and it is natural to ask whether one can classify BPS black holes. Asymptotically-flat BPS black-hole solutions are known only for $d=4,5$.

In addition to rendering microscopic computations tractable, the additional ingredient of supersymmetry makes the classification of black holes easier. A supersymmetric solution admits a

Living Reviews in Relativity

http://www . livingreviews . org/lrr-2008-6 
globally-defined super-covariantly constant spinor (see, e.g., [107]). This is such a strong constraint that it is often possible to determine the general solution with this property. This was first done for minimal $d=4, N=2$ supergravity, whose bosonic sector is Einstein-Maxwell theory. It can be shown [237] that any supersymmetric solution of this theory must be either a pp-wave or a member of the well-known Israel-Wilson-Perjes family of solutions (see, e.g., [228]). The only black-hole solutions in the IWP family are believed to be the multi-Reissner-Nordström solutions [131], and this can be proved subject to one assumption ${ }^{9}$ [45]. Hence, this is a uniqueness theorem for supersymmetric black-hole solutions of minimal $d=4, N=2$ supergravity.

This success has been partially extended to minimal $d=5$ supergravity. It can be shown that any supersymmetric black hole must have near-horizon geometry locally isometric to either (i) the near-horizon geometry of the BMPV black hole, (ii) $A d S_{3} \times S^{2}$, or (iii) flat space [216]. Case (iii) would give a black hole with $T^{3}$ horizon, which seems unlikely in view of the black-hole topology theorem discussed above (although this does not cover supersymmetric black holes, since they are necessarily extremal). An explicit form for supersymmetric solutions of this theory is known [101] and can be exploited, together with an additional assumption ${ }^{10}$, to show that the only black hole of type (i) is the BMPV black hole itself. The supersymmetric black ring of [74] belongs to class (ii). The remaining step required to complete the classification would be to prove that this is the only solution in this class. These results can be extended to minimal $d=5$ supergravity coupled to vector multiplets [123].

These results show that much more is known about $d=5$ supersymmetric black holes than about more general stationary $d=5$ black holes. Note that no assumptions about the topology of the horizon, or the number of rotational symmetries are required to obtain these results; they are outputs, not inputs. One might interpret this as weak evidence that, for general $d=5$ black holes, topologies other than $S^{3}$ and $S^{1} \times S^{2}$ cannot occur and that the assumption of two rotational symmetries is reasonable. However, one should be cautious in generalizing from BPS to non-BPS black holes, since it is known that many properties of the former (e.g., stability) do not always generalize to the latter.

\subsection{Algebraic classification}

In $d=4$ dimensions, spacetimes can be classified according to the algebraic type of the Weyl tensor. Associated with the Weyl tensor are four "principal null vectors" [244]. In general these are distinct, but two or more of them coincide in an algebraically special spacetime. For example, the Kerr-Newman spacetime is type D, which means that it has two pairs of identical principal null vectors.

Given that known $d=4$ black-hole solutions are algebraically special, it is natural to investigate whether the same is true in $d>4$ dimensions. Before doing this, it is necessary to classify possible algebraic types of the Weyl tensor in higher dimensions. This can be done using a spinorial approach for the special case of $d=5$ [65]. The formalism for all dimensions $d \geq 4$ has been developed in [50] (and reviewed in [51]). It is based on "aligned null directions", which generalize the concept of principal null vectors in $d=4$. A general $d>4$ spacetime admits no aligned null directions. The Weyl tensor is said to be algebraically special if one or more aligned null directions exist.

The algebraic types of some higher-dimensional black-hole solutions have been determined. The Myers-Perry black hole belongs to the higher-dimensional generalization of the $d=4$ type $\mathrm{D}$ class $[66,213,215]$. The black ring is also known to be algebraically special, although not as

\footnotetext{
${ }^{9}$ Supersymmetric solutions admit a globally defined Killing vector field that is timelike or null. The assumption is that it is non-null everywhere outside the horizon.

${ }^{10}$ The same assumption as for the $d=4, N=2$ case just discussed.
} 
special as the Myers-Perry black hole [213, 215]. Further analysis of the Weyl tensor and principal null congruences in higher dimensions can be found in [214].

In $d=4$ dimensions, interesting new solutions (e.g., the spinning C-metric) were discovered by solving the Einstein equations to determine all solutions of type D [159]. Perhaps the same strategy would be fruitful in higher dimensions. A particular class of spacetimes, namely RobinsonTrautman, admitting a hypersurface-orthogonal, nonshearing and expanding geodesic null congruence, has been studied in [209]. However, unlike in four dimensions, this class does not contain the analogue of the C-metric.

\subsection{Laws of black-hole mechanics}

The laws of black-hole mechanics are generally valid in any number of dimensions. The only novelty arises in the first law due to new possibilities afforded by novel black holes. A nontrivial extension is to include dipoles charges that are independent of the conserved charges. An explicit calculation in [79] shows that black rings with a dipole satisfy a form of the first law with an additional 'work' term $\phi d q$, where $q$ is the dipole charge and $\phi$ its respective potential. The appearance of the dipole here was surprising, since the most general derivation of the first law seems to allow only conserved charges into it. However, [52] shows that a new surface term enters due to the impossibility of globally defining the dipole potential $\phi$ in such a way that it is simultaneously regular at the rotation axis and at the horizon. Then one finds

$$
d M=\frac{\kappa}{8 \pi} \mathcal{A}_{H}+\Omega_{H} d J+\Phi d Q+\phi d q,
$$

where $Q$ and $\Phi$ are the conserved charge and its potential, respectively (see also [6]).

The next extension is not truly specific to $d>4$, but it refers to a situation for which there are no known four-dimensional examples: stationary multiple-black-hole solutions with nondegenerate horizons. As we have seen, there are plenty of these in $d \geq 5$. In this case, the first law can be easily shown to take the form [73]

$$
d M=\sum_{i}\left(\frac{\kappa^{(i)}}{8 \pi} d \mathcal{A}_{H}^{(i)}+\Omega_{j}^{(i)} d J_{j}^{(i)}+\Phi^{(i)} d Q^{(i)}\right) .
$$

Here the index $i$ labels the different disconnected components of the event horizon and $j$ their independent angular momenta. The Komar angular momentum $J_{j}^{(i)}$ and the charge $Q^{(i)}$ are computed as integrals on a surface that encloses a single component of the horizon, generated by the vector $\partial_{t}+\Omega_{j}^{(i)} \partial_{\phi_{j}}$. The potential $\Phi^{(i)}$ is the difference between the potential at infinity and the potential on the $i$-th component of the horizon; in general we cannot choose a globally defined gauge potential that simultaneously vanishes on all horizon components. Presumably the result can be extended to include dipoles but the possible subtleties have not been dealt with yet. A Smarr relation also exists that relates the total ADM mass to the sums of the different 'heat' and 'work' terms on each horizon component [73].

\subsection{Hawking radiation and black-hole thermodynamics}

The extension of Hawking's original calculation to most of the black holes that we have discussed in this review presents several difficulties, but we regard this as mostly a technical issue. In our opinion, there is no physically reasonable objection to the expectation that Hawking radiation is essentially unmodified in higher dimensions; a black hole emits radiation that, up to grey-body corrections, has a Planckian spectrum of temperature $T=\kappa / 2 \pi$ and chemical potentials $\Omega$, $\Phi$, etc.

Some of the technical difficulties relate to the problem of wave propagation in the black-hole background; this can only be dealt with analytically for Myers-Perry black holes, since only in this

Living Reviews in Relativity

http://www.livingreviews.org/lrr-2008-6 
case have the variables been separated (and then only for scalars and vectors in the general rotating case). There is in fact a considerable body of literature on the problem of radiation from MP black holes, largely motivated by their possible detection in scenarios with large extra dimensions. As mentioned in Section 2, this is outside the scope of this paper and we refer to [30, 155] for reviews.

There are also peculiarities with wave propagation that depend on the parity of the number of dimensions [206, 24], but these are unlikely to modify in any essential way the Planckian spectrum of radiation. This is, in fact, confirmed by detailed microscopic derivations of Hawking radiation in five dimensions based on string theory [188]. Other approaches to Hawking radiation that do not require one to analyze wave propagation have been applied to black rings [193], confirming the expected results. An early result was the analysis of vacuum polarization in higher-dimensional black holes in [92]. More recently, [204] claims that the evolution of a five-dimensional rotating black hole emitting scalar Hawking radiation leads, for arbitrary initial values of the two rotation parameters $a$ and $b$, to a fixed asymptotic value of $a / M^{2}=b / M^{2}=$ const $\neq 0$.

The spectrum of radiation from a multiple-black-hole configuration will contain several components with parameters $\left(T^{(i)}, \Omega^{(i)}, \Phi^{(i)}, \ldots\right)$, so it will not really be a thermal distribution unless all the black holes have equal intensive parameters. This is, of course, the conventional condition for thermal equilibrium.

The Euclidean formulation of black-hole thermodynamics remains largely the same as in four dimensions. For rotating solutions, it is more convenient not to continue analytically the angular velocities and instead to work with complex sections that have real actions. In fact, black rings do not admit nonsingular real Euclidean sections [76]. Multiple-black-hole configurations with disconnected components of the horizon with different surface gravities, angular velocities, and electric potentials clearly do not admit regular Euclidean sections. Still, the Euclidean periodicity of the horizon generator formally yields the horizon temperature in the usual fashion.

\subsection{Apparent and isolated horizons and critical phenomena}

A number of other interesting studies of higher-dimensional black holes have been made. The properties of higher-dimensional apparent horizons have been analyzed in [223], which provides simple criteria to determine them. The isoperimetric inequalities and the hoop conjecture, concerning bounds on the sizes of apparent horizons through the mass they enclose, involve new features in higher dimensions. For instance, the four-dimensional hoop conjecture posits that a necessary and sufficient condition for the formation of a black hole is that a mass $M$ gets compacted into a region whose circumference in every direction is $\mathcal{C} \leq 4 \pi G M$ [236]. A generalization of this conjecture to $d>4$ using a hoop of spatial dimension 1 , in the form $\mathcal{C} \leq \#(G M)^{1 /(d-3)}$, is unfeasible; the existence of black objects whose horizons have arbitrary extent in some directions (e.g., black strings, black rings, and ultraspinning black holes) shows that this condition is not necessary. It seems possible, however, that plausible, necessary, and sufficient conditions exist using the area of hoops of spatial codimension $d-3$ in the form $\mathcal{C}_{d-3} \leq \# G M$, although black rings may require hoops with nonspherical topology $[145,8]$. There is also some evidence that the isoperimetric inequalities, which bound the spatial area of the apparent horizon by the mass that it encloses [208], may be extended in the form $\mathcal{A} \leq \#(G M)^{\frac{d-2}{d-3}}[145,8]$. See [257, 256, 224] for further work on these subjects.

The study of possible topologies for black-hole event horizons may be helped by the study of possible apparent horizons in initial data sets. [222] shows that it is possible to construct time-symmetric initial-data sets for black holes with apparent-horizon topology with the form of a product of spheres. Time symmetry, however, implies that these apparent horizons cannot correspond to rotating black holes and it is likely that their evolution in time leads to collapse into a spherical horizon.

The formalism of isolated horizons and the laws of black-hole mechanics for them, have been 
extended to higher dimensions in [182, 170], and then to five-dimensional Einstein-Maxwell theory with the Chern-Simons term [183] and anti-de Sitter asymptotics [5].

Finally, the critical phenomena in the collapse of a massless scalar at the onset of black-hole formation, discovered by Choptuik [41], have been studied in [227]. 


\section{Solutions with a Cosmological Constant}

\subsection{Motivation}

The motivation for considering higher-dimensional black holes with a cosmological constant arises from the AdS/CFT correspondence [1]. This is an equivalence between string theory on spacetimes asymptotic to $A d S_{d} \times X$, where $X$ is a compact manifold, and a conformal field theory (CFT) defined on the Einstein universe $R \times S^{d-2}$, which is the conformal boundary of $A d S_{d}$. The best understood example is the case of type IIB string theory on spacetimes asymptotic to $\operatorname{Ad} S_{5} \times S^{5}$, which is dual to $\mathcal{N}=4 S U(N)$ super-Yang-Mills theory on $R \times S^{3}$. Type IIB string theory can be replaced by IIB supergravity in the limit of large $N$ and strong 't Hooft coupling in the Yang-Mills theory.

Most studies of black holes in the AdS/CFT correspondence involve dimensional reduction on $X$ to obtain a $d$-dimensional gauged supergravity theory with a negative cosmological constant. For example, one can reduce type IIB supergravity on $S^{5}$ to obtain $d=5, \mathcal{N}=4 S O(6)$ gauged supergravity. One then seeks asymptotically anti-de Sitter black-hole solutions of the gauged supergravity theory. This is certainly easier than trying to find solutions in ten or eleven dimensions. However, one should bear in mind that there may exist asymptotically $A d S_{d} \times X$ black-hole solutions that cannot be dimensionally reduced to $d$ dimensions. Such solutions would not be discovered using gauged supergravity.

In this section we shall discuss asymptotically $A d S_{d}$ black-hole solutions of the $d=4,5,6,7$ gauged supergravity theories arising from the reduction of $d=10$ or $d=11$ supergravity on spheres. The emphasis will be on classical properties of the solutions rather than their implications for CFT. In AdS, linearized supergravity perturbations can be classified as normalizable or non-normalizable according to how they behave near infinity [1]. By "asymptotically AdS" we mean that we are restricting ourselves to considering solutions that approach a normalizable deformation of global AdS near infinity. A non-normalizable perturbation would correspond to a deformation of the CFT, for instance, making it nonconformal. Black-hole solutions with such asymptotics have been constructed, but space prevents us from considering them here.

\subsection{Schwarzschild-AdS}

The simplest example of an asymptotically AdS black hole is the Schwarzschild-AdS solution [172, 250]:

$$
d s^{2}=-U(r) d t^{2}+U(r)^{-1} d r^{2}+r^{2} d \Omega_{d-2}^{2}, \quad U(r)=1-\frac{\mu}{r^{d-3}}+\frac{r^{2}}{\ell^{2}},
$$

where $\mu$ is proportional to the mass, and $\ell$ is the radius of curvature of the AdS ground state ${ }^{11}$. The solution has a regular horizon for any $\mu>0$. Definitions of mass and angular momentum for asymptotically AdS spacetimes have been given in $d=4$ [4] and $d \geq 4$ [3]. The mass of Schwarzschild-AdS relative to the AdS ground state is [250]

$$
M=\frac{(d-2) \Omega_{d-2} \mu}{16 \pi G} .
$$

For $d=4$, the stability of Schwarzschild-AdS against linearized gravitational perturbations has been proven in [163]. For $d>4$, spherical symmetry enables one to decompose linearized gravitational perturbations into scalar/vector/tensor types. The equations governing each type can be reduced to ODEs of Schrödinger form, and the stability of vector and tensor perturbations can

\footnotetext{
${ }^{11}$ The "topological black holes" with $U(r)=k-\frac{\mu}{r^{d-3}}+\frac{r^{2}}{\ell^{2}}, k=0,-1$ and toroidal or hyperbolic horizons [15] are excluded from our review by their asymptotics.
} 
be established [151]. Stability with respect to scalar gravitational perturbations has not yet been established.

It is expected that the Schwarzschild-AdS black hole is the unique, static, asymptotically AdS, black-hole solution of vacuum gravity with a negative cosmological constant, but this has not been proven.

The thermodynamics of Schwarzschild-AdS were discussed by Hawking and Page for $d=4$ [134] and Witten for $d>4$ [250]. Let $r_{+}$denote the horizon radius of the solution. For a small black hole, $r_{+} \ll \ell$, the thermodynamic properties are qualitatively similar to those of an asymptotically-flat Schwarzschild black hole, i.e., the temperature decreases with increasing $r_{+}$so the heat capacity of the hole is negative (as $r_{+}$is a monotonic function of $\mu$ ). However, there is an intermediate value of $r_{+} \sim \ell$ at which the temperature reaches a global minimum $T_{\min }$ and then becomes an increasing function of $r_{+}$. Hence the heat capacity of large black holes is positive. This implies that the black hole can reach a stable equilibrium with its own radiation (which is confined near the hole by the gravitational potential $\sim r^{2} / \ell^{2}$ at large $r$ ). Note that for $T>T_{\min }$ there are two black-hole solutions with the same temperature: a large one with positive specific heat and a small one with negative specific heat.

These properties lead to an interesting phase structure for gravity in AdS [134]. At low temperature, $T<T_{\min }$, there is no black-hole solution and the preferred phase is thermal radiation in AdS. At $T \sim T_{\min }$, black holes exists but have greater free energy than thermal radiation. However, there is a critical temperature $T_{\mathrm{HP}}>T_{\min }$ beyond which the large black hole has lower free energy than thermal radiation and the small black hole. The interpretation is that the canonical ensemble for gravity in AdS exhibits a (first-order) phase transition at $T=T_{\min }$.

In the AdS/CFT context, this Hawking-Page phase transition is interpreted as the gravitational description of a thermal phase transition of the (strongly coupled) CFT on the Einstein universe [249, 250].

When oxidized, to ten or eleven dimensions, the radius $r_{+}$of a small Schwarzschild-AdS black hole will be much less than the radius of curvature $(\sim \ell)$ of the internal space $X$. This suggests that the black hole will suffer from a classical Gregory-Laflamme-type instability. The probable endpoint of the instability would be a small black hole localized on $X$, and therefore would not admit a description in gauged supergravity. Since the radius of curvature of $X$ is typically $\ell$ and the black hole is much smaller than $\ell$, the geometry near the hole should be well approximated by the ten or eleven-dimensional Schwarzschild solution (see e.g., [141]). However, an exact solution of this form is not known.

\subsection{Stationary vacuum solutions}

If we consider pure gravity with a negative cosmological constant then the most general known family of asymptotically-AdS black-hole solutions is the generalization of the Kerr-Myers-Perry solutions to include a cosmological constant. It seems likely that black rings would exist in asymptotically-AdS spacetimes, but no exact solutions are known. ${ }^{12}$

The $d=4$ Kerr-AdS solution was constructed long ago [27]. It can be parameterized by its mass $M$ and angular momentum $J$, which have been calculated (using the definitions of [4]) in [113]. The region of the $(M, J)$ plane covered by these black holes is shown in Figure 13. Note that, in AdS, angular momentum is a central charge [108]. Hence regular vacuum solutions exhibit a nontrivial lower bound on their mass: $M \geq|J| / \ell$. The Kerr-AdS solution never saturates this bound.

The Myers-Perry-AdS solution was obtained in [133] for $d=5$ and for $d>5$ with rotation in a single plane. The general $d>5$ solution was obtained in [111, 112]. They have horizons

\footnotetext{
${ }^{12}$ Note that topological censorship can be used to exclude the existence of topologically nonspherical black holes in $\mathrm{AdS}_{4}$ [97].
}

Living Reviews in Relativity

http://www. livingreviews.org/lrr-2008-6 


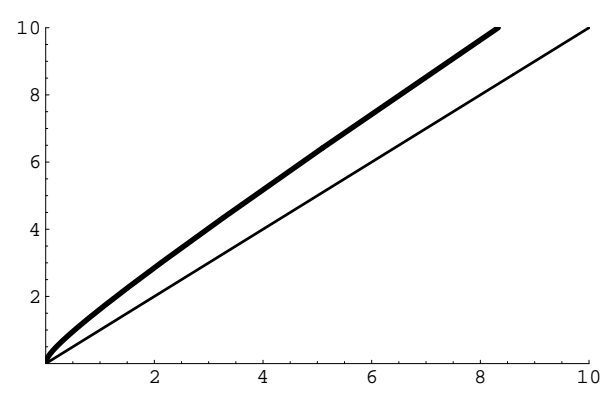

Figure 13: $G M / \ell$ against $G|J| / \ell^{2}$ for $d=4$ Kerr-AdS black holes. The thick curve corresponds to extremal black holes. Black-hole solutions lie on, or above, this curve (which was determined using results in [20]). The thin line is the BPS bound $M=|J| / \ell$.

of spherical topology. There is some confusion in the literature concerning the conserved charges carried by these solutions. A careful discussion can be found in [113]. The solutions are uniquely specified by their mass and angular momenta. For $d=5$, the region of $\left(M, J_{1}, J_{2}\right)$-space covered by the Myers-Perry-AdS solution is shown in Figure 14.

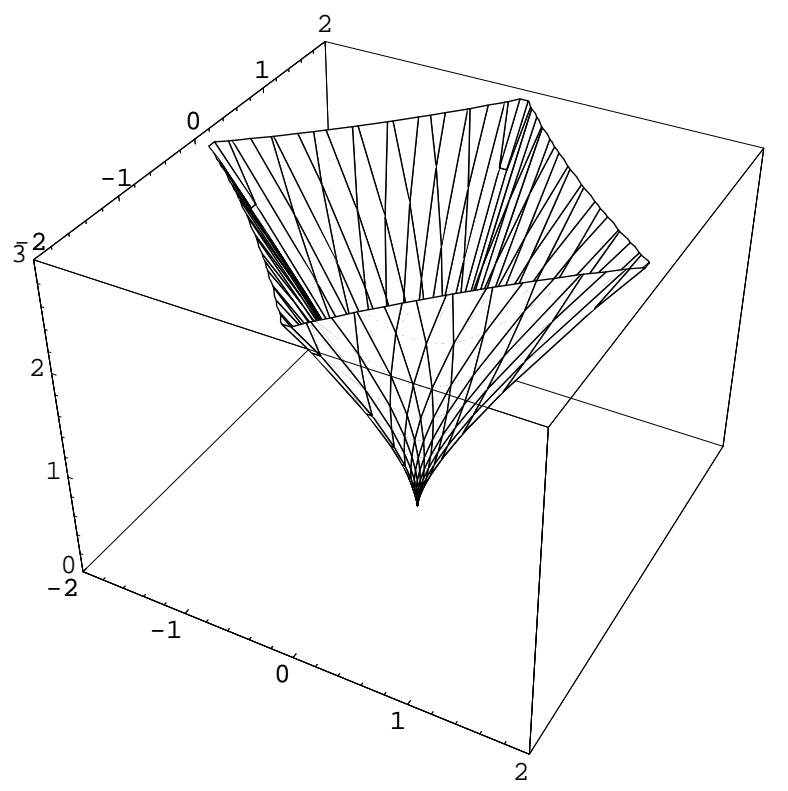

Figure 14: $G M / \ell^{2}$ (vertical) against $G\left|J_{i}\right| / \ell^{3}$ for $d=5$ Myers-Perry-AdS black holes. Nonextremal black holes fill the region above the surface. The surface corresponds to extremal black holes, except when one of the angular momenta vanishes (in which case there is not a regular horizon, just as in the asymptotically-flat case). This "extremal surface" lies inside the square-based pyramid (with vertex at the origin) defined by the BPS relation $M=\left|J_{1}\right| / \ell+\left|J_{2}\right| / \ell$, so none of the black holes are BPS.

Kerr-Myers-Perry-AdS solutions have the same symmetries as their asymptotically-flat cousins, and exhibit similar enhancement of symmetry in special cases. The integrability of the geodesic equation and separability of the Klein-Gordon equation also extends to this case [207, 173, 90].

These solutions reduce to the Schwarzschild-AdS solution in the limit of zero angular momentum. It has been shown that the only regular stationary perturbations of the Schwarzschild-AdS solution are those that correspond to taking infinitesimal angular momenta in these rotating so- 
lutions [162]. Hence, if other stationary vacuum black-hole solutions exist (e.g., black rings) then they are not continuously connected to the Schwarzschild-AdS solution.

These solutions exhibit an important qualitative difference from their asymptotically-flat cousins. Consider the Killing field tangent to the null-geodesic generators of the horizon:

$$
V=\frac{\partial}{\partial t}+\Omega_{i} \frac{\partial}{\partial \phi_{i}}
$$

In asymptotically-flat spacetime, this Killing field is spacelike far from the black hole, which implies that it is impossible for matter to co-rotate rigidly with the hole (i.e., to move on orbits of $V$ ). However, in AdS, if $\Omega_{i} \ell \leq 1$ then $V$ is timelike everywhere outside the horizon. This implies that rigid co-rotation is possible; the Killing field $V$ defines a co-rotating reference frame. Consequently, there exists a Hartle-Hawking state describing thermal equilibrium of the black hole with corotating thermal radiation [133].

The dual CFT interpretation is of CFT matter in thermal equilibrium rotating around the Einstein universe [133]. There is an interesting phase structure, generalizing that found for Schwarzschild-AdS [133, 13, 20, 135]. For sufficiently large black holes, one can study the dual CFT using a fluid mechanics approximation, which gives quantitative agreement with black-hole thermodynamics [14].

What happens if $\Omega_{i} \ell>1$ ? Such black holes are believed to be classically unstable. It was observed in [135] that rotating black holes in AdS may suffer from a super-radiant instability, in which energy and angular momentum are extracted from the black hole by super-radiant modes. However, it was proven that this cannot occur if $\Omega_{i} \ell \leq 1$. But if $\Omega_{i} \ell>1$ then an instability may be present. This makes sense from a dual CFT perspective; configurations with $\Omega_{i} \ell>1$ would correspond to CFT matter rotating faster than light in the Einstein universe [133]. The existence of an instability was first demonstrated for small $d=4$ Kerr-AdS black holes in [23]. A general analysis of odd-dimensional black holes with equal angular momenta reveals that the threshold of instability is at $\Omega_{i} \ell=1$ [177], i.e., precisely where the stability argument of [135] fails. The endpoint of this classical bulk instability is not known.

In $d=4$, Figure 13 reveals (using $\Omega=d M / d J$ ) that all extremal Kerr-AdS black holes have $\Omega \ell>1$ and are, therefore, expected to be unstable. We have checked that $d=5$ extremal MyersPerry-AdS black holes also have $\Omega_{i} \ell>1$ and so they too should be classically unstable. However, the instability should be very slow when the black-hole size is much smaller than the AdS radius $\ell$, and one expects it to disappear as $\ell \rightarrow \infty$ : it takes an increasingly long time for the super-radiant modes to bounce back off the AdS boundary.

Finally, we should mention a subtlety concerning the use of the term "stationary" in asymptotically AdS spacetimes [177]. Consider the $A d S_{5}$ metric

$$
d s^{2}=-\left(1+\frac{r^{2}}{\ell^{2}}\right) d t^{2}+\left(1+\frac{r^{2}}{\ell^{2}}\right)^{-1} d r^{2}+r^{2}\left(d \theta^{2}+\sin ^{2} \theta d \phi^{2}+\cos ^{2} \theta d \psi^{2}\right)
$$

This admits several types of globally-timelike Killing fields. For example, there is the "usual" generator of time translations $k=\partial / \partial t$, which has unbounded norm, but there is also the "rotating" Killing field $V=\partial / \partial t+\ell^{-1} \partial / \partial \phi+\ell^{-1} \partial / \partial \psi$, which has constant norm. On the conformal boundary, $k$ is timelike and $V$ is null. Hence, from a boundary perspective, particles following orbits of $V$ are rotating at the speed of light. These two different types of timelike Killing vector field allow one to define two distinct notions of stationarity for asymptotically-AdS spacetimes. So far, all known black hole solutions are stationary with respect to both definitions because they admit global Killing fields analogous to $\partial / \partial \phi, \partial / \partial \psi$. However, it is conceivable that there exist AdS black holes (with less symmetry than known solutions) that are stationary only with respect to the second definition, i.e., they admit a Killing field that behaves asymptotically like $V$ but not

Living Reviews in Relativity

http://www. livingreviews.org//rr-2008-6 
one behaving asymptotically like $k$. From a boundary CFT perspective, such black holes would rotate at the speed of light.

\subsection{Gauged supergravity theories}

In order to discuss charged anti-de Sitter black holes we will need to specify which gauged supergravity theories we are interested in. The best-understood examples arise from the dimensional reduction of $d=10$ or $d=11$ dimensional supergravity theories on spheres to give theories with maximal supersymmetry and non-Abelian gauge groups. However, most work on constructing explicit black hole solutions has dealt with consistent truncations of these theories, with reduced supersymmetry, in which the non-Abelian gauge group is replaced by its maximal Abelian subgroup. Indeed, there is no known black-hole solution with a nontrivial non-Abelian gauge field obeying normalizable boundary conditions.

There is a consistent dimensional reduction of $d=11$ supergravity on $S^{7}$ to give $d=4, \mathcal{N}=8$, $S O(8)$ gauged supergravity [67]. This non-Abelian theory can be consistently truncated to give $d=4, \mathcal{N}=2, U(1)^{4}$ gauged supergravity, whose bosonic sector is Einstein gravity coupled to four Maxwell fields and three complex scalars [55]. The scalar potential is negative at its global maximum. The $A d S_{4}$ ground state of the theory has the scalars taking constant values at this maximum. One can truncate this theory further by taking the scalars to sit at the top of their potential, and setting the Maxwell fields equal to each other. This gives minimal $d=4, \mathcal{N}=2$ gauged supergravity, whose bosonic sector is Einstein-Maxwell theory with a cosmological constant. The embedding of minimal $d=4, \mathcal{N}=2$ gauged supergravity theories into $d=11$ supergravity can be given explicitly [31], and is much simpler than the embedding of the non-Abelian $\mathcal{N}=8$ theory.

The $d=11$ supergravity theory can also be dimensionally reduced on $S^{4}$ to give $d=7, \mathcal{N}=2$, $S O(5)$ gauged supergravity [201, 202].

The $d=10$ massive IIA supergravity can be dimensionally reduced on $S^{4}$ to give $d=6 \mathcal{N}=2$ $S U(2)$ gauged supergravity [59]. This theory has half-maximal supersymmetry.

It is believed that the $d=10$ type IIB supergravity theory can be consistently reduced on $S^{5}$ to give $d=5, \mathcal{N}=4, S O(6)$ gauged supergravity, although this has been established only for a subsector of the full theory [62]. This theory can be truncated further to give $d=5, \mathcal{N}=1, U(1)^{3}$ gauged supergravity with three vectors and two scalars. Again, setting the scalars to constants and making the vectors equal gives the minimal $d=5$ gauged supergravity, whose bosonic sector is Einstein-Maxwell theory with a negative cosmological constant and a Chern-Simons coupling. The explicit embeddings of these Abelian theories into $d=10$ type IIB supergravity are known [31, 55].

It is sometimes possible to obtain a given lower-dimensional supergravity theory from several different compactifications of a higher-dimensional theory. For example, minimal $d=5$ gauged supergravity can be obtained by compactifying type IIB supergravity on any Sasaki-Einstein space $Y^{p, q}[18]$. More generally, if there is a supersymmetric solution of type IIB supergravity that is a warped product of $A d S_{5}$ with some compact manifold $X_{5}$, then type IIB supergravity can be dimensionally reduced on $X_{5}$ to give minimal $d=5$ gauged supergravity [104]. An analogous statement holds for compactifications of $d=11$ supergravity to give minimal $\mathcal{N}=2, d=4$ gauged supergravity or minimal $d=5$ gauged supergravity [103, 104].

\subsection{Static charged solutions}

The $d=4$ Reissner-Nordström-AdS black hole is a solution of minimal $\mathcal{N}=2$ gauged supergravity. It is parameterized by its mass $M$ and electric and magnetic charges $Q, P$. This solution is stable against linearized perturbations within this (Einstein-Maxwell) theory [164]. Compared with its asymptotically-flat counterpart, perhaps the most surprising feature of this solution is that it never 
saturates a BPS bound. If the mass of the black hole is lowered, it will eventually become extremal, but the extremal solution is not BPS. If one imposes the BPS condition on the solution, then one obtains a naked singularity rather than a black hole [221, 185].

Static, spherically-symmetric, charged, black-hole solutions of the $\mathcal{N}=2, d=4, U(1)^{4}$ gauged supergravity theory were obtained in [69]. The solutions carry only electric charges and are parameterized by their mass $M$ and electric charges $Q_{i}$. Alternatively they can be dualized to give purely magnetic solutions. Once again, they never saturate a BPS bound. One would expect the existence of dyonic solutions of this theory, but such solutions have not been constructed.

Static, spherically-symmetric, charged, black-hole solutions of $d=5, U(1)^{3}$ gauged supergravity were obtained in [9]. They are parameterized by their mass $M$ and electric charges $Q_{i}$. If the charges are set equal to each other then one recovers the $d=5$ Reissner-Nordström solution of minimal $d=5$ gauged supergravity. The solutions never saturate a BPS bound.

A static, spherically-symmetric, charged black-hole solution of $d=6, S U(2)$ gauged supergravity was given in [59]. Only a single Abelian component of the gauge field is excited, and the solution is parameterized by its charge and mass.

Static, spherically-symmetric, charged, black-hole solutions of $d=7, S O(5)$ gauged supergravity are known [55]. They can be embedded into a truncated version of the full theory in which there are two Abelian vectors and two scalars. They are parameterized by their mass and electric charges.

Electrically-charged, asymptotically-AdS, black-hole solutions exhibit a Hawking-Page like phase transition in the bulk, which entails a corresponding phase transition for the dual CFT at finite temperature in the presence of chemical potentials for the R-charge. This has been studied in $[31,56,57,32]$.

These black holes exhibit an interesting instability [121, 122]. This is best understood for a black hole so large (compared to the AdS radius) that the curvature of its horizon can be neglected, i.e., it can be approximated by a black brane. The dual CFT interpretation is as a finite temperature configuration in flat space with finite charge density. For certain regions of parameter space, it turns out that the entropy increases if the charge density becomes nonuniform (with the total charge and energy held fixed). Hence, the thermal CFT state exhibits an instability. Using the AdS/CFT dictionary, this maps to a classical instability in the bulk in which the horizon becomes translationally nonuniform, i.e., a Gregory-Laflamme instability. The remarkable feature of this argument is that it reveals that a classical Gregory-Laflamme instability should be present precisely when the black brane becomes locally thermodynamically unstable. Here, local thermodynamic stability means having an entropy, which is concave down as a function of the energy and other conserved charges (if the only conserved charge is the energy, then this is equivalent to positivity of the heat capacity). The Gubser-Mitra (or "correlated stability") conjecture asserts that this correspondence should apply to any black brane, not just asymptotically-AdS solutions. See [128] for more discussion of this correspondence.

For finite-radius black holes, the argument is not so clear cut because the dual CFT lives in the Einstein universe rather than flat spacetime, so finite size effects will affect the CFT argument and the Gubser-Mitra conjecture does not apply. Nevertheless, it should be a good approximation for sufficiently large black holes and hence there will be a certain range of parameters for which large charged black holes are classically unstable. ${ }^{13}$

\subsection{Stationary charged solutions}

The most general, known, stationary, black-hole solution of minimal $d=4, \mathcal{N}=2$ gauged supergravity is the Kerr-Newman-AdS solution, which is uniquely parameterized by its mass $M$, angular

\footnotetext{
${ }^{13}$ Note that this does not disagree with the stability result of [164] for $d=4$ Reissner-Nordström-AdS since the instability involves scalar fields and hence cannot be seen within minimal $\mathcal{N}=2$ gauged supergravity.
}

Living Reviews in Relativity

http://www . livingreviews . org//rr-2008-6 
momentum $J$ and electric and magnetic charges $(Q, P)$. The thermodynamic properties of this solution, and implications for the dual CFT were investigated in [20]. An important property of this solution is that it can preserve some supersymmetry. This occurs for a one-parameter subfamily specified by the electric charge: $M=M(Q), J(Q), P=0[171,21]$. Hence supersymmetric black holes can exist in AdS but they exhibit an important qualitative difference from the asymptotically flat case; they must rotate.

Charged rotating black-hole solutions of more general $d=4$ gauged supergravity theories, e.g., $\mathcal{N}=2, U(1)^{4}$ gauged supergravity, should also exist. Electrically charged, rotating solutions of the $U(1)^{4}$ theory, with the four charges set pairwise equal, were constructed in [36].

Charged, rotating black-hole solutions of $d=7, S O(5)$ gauged supergravity have been constructed by truncating to a $U(1)^{2}$ theory [40, 43]. In this theory, one expects the existence of a topologically-spherical black-hole solution parameterized by its mass, three angular momenta, and two electric charges. This general solution is not yet known. However, solutions with three equal angular momenta but unequal charges have been constructed [40], as have solutions with equal charges but unequal angular momenta [43]. Both types of solution admit BPS limits.

Charged, rotating black-hole solutions of $d=6$ gauged supergravity have not yet been constructed.

The construction of charged rotating black-hole solutions of $d=5$ gauged supergravity has attracted more attention $[125,124,60,61,37,38,178,39,191]$. The most general known blackhole solution of the minimal theory is that of [38]. This solution is parameterized by the conserved charges of the theory, i.e., the mass $M$, electric charge $Q$ and two angular momenta $J_{1}, J_{2}$. Intuition based on results proved for asymptotically-flat solutions suggests that, for this theory, this is the most general topologically-spherical stationary black hole with two rotational symmetries. In the BPS limit, these solutions reduce to a two-parameter family of supersymmetric black holes. In other words, one loses two parameters in the BPS limit (just as for nonstatic asymptotically-flat black holes in $d=5$, e.g., the BMPV black hole or BPS black rings).

Analogous solutions of $d=5, U(1)^{3}$ gauged supergravity are expected to be parameterized by the six conserved quantities $M, J_{1}, J_{2}, Q_{1}, Q_{2}, Q_{3}$. However, a six-parameter solution is not yet known. The most general known solutions are the four-parameter BPS solution of [178], and the five-parameter nonextremal solution of [191], which has two of the charges $Q_{i}$ equal. The former is expected to be the general BPS limit of the yet to be discovered six-parameter black-hole solution (as one expects to lose two parameters in the BPS limit). The latter solution should be obtained from the general six-parameter solution by setting two of the charges equal.

Supersymmetric AdS black holes have $\Omega_{i} \ell=1$, which implies that they rotate at the speed of light with respect to the conformal boundary [125]. More precisely, the co-rotating Killing field becomes null on the conformal boundary. Hence, the CFT interpretation of these black holes involves matter rotating at the speed of light in the Einstein universe. The main motivation for studying supersymmetric AdS black holes is the expectation that it should be possible to perform a microscopic CFT calculation of their entropy. The idea is to count states in weakly coupled CFT and then extrapolate to strong coupling. In doing this, one has to count only states in short BPS multiplets that do not combine into long multiplets as the coupling is increased. One way of trying to do this is to work with an index that receives vanishing contributions from states in multiplets that can combine into long multiplets. Unfortunately, such indices do not give agreement with black-hole entropy [160]. This is not a contradiction; although certain multiplets may have the right quantum numbers to combine into a long multiplet, the dynamics of the theory may prevent this from occurring, so the index undercounts BPS states.

The fact that these supersymmetric black holes have only four independent parameters is puzzling from the CFT perspective, since BPS states in the CFT carry five independent charges. Maybe there are more general black-hole solutions. It seems unlikely that one could generalize the solutions of [178] to include an extra parameter since then one would also have an extra parameter, 
in the corresponding non-BPS solutions, which would therefore form a seven parameter family in a theory with only six conserved charges. This seems unlikely for topologically-spherical black holes. But we know that black rings can carry nonconserved charges, so maybe this points to the existence of supersymmetric AdS black rings. However, it has been shown that such solutions do not exist in minimal $d=5$ gauged supergravity [179]. The proof involves classifying supersymmetric nearhorizon geometries (with two rotational symmetries), and showing that $S^{1} \times S^{2}$ topology horizons always suffer from a conical singularity, except in the limit in which the cosmological constant vanishes. Analogous results for the $U(1)^{3}$ theory have also been obtained [176]. So if AdS black rings exist then they cannot be "balanced" in the BPS limit.

Maybe the resolution of the puzzle involves $10 \mathrm{~d}$ black holes with no $5 \mathrm{D}$ interpretation, or 5D black holes involving non-Abelian gauge fields, or 5D black holes with only one rotational symmetry. Alternatively, perhaps we already know all the BPS black-hole solutions and the puzzle arises from a lack of understanding of the CFT. For example, maybe, at strong coupling, only a four charge subspace of BPS CFT states carries enough entropy to correspond to a macroscopic black hole. 


\section{Acknowledgments}

RE is supported in part by DURSI 2005 SGR 00082, CICYT FPA 2004-04582-C02-02 and FPA 2007-66665C02-02, and the European Community FP6 program MRTN-CT-2004-005104. HSR is a Royal Society University Research Fellow. 


\section{References}

[1] Aharony, O., Gubser, S.S., Maldacena, J.M., Ooguri, H., and Oz, Y., "Large N field theories, string theory and gravity", Phys. Rep., 323, 183-386, (2000). Related online version (cited on 14 February 2008):

http://arXiv.org/abs/hep-th/9905111. 1, 2.1, 9.1

[2] Arcioni, G., and Lozano-Tellechea, E., "Stability and critical phenomena of black holes and black rings", Phys. Rev. D, 72, 104021, (2005). Related online version (cited on 14 February 2008):

http://arXiv.org/abs/hep-th/0412118. 5.4

[3] Ashtekar, A., and Das, S., "Asymptotically anti-de Sitter space-times: Conserved quantities", Class. Quantum Grav., 17, L17, (2000). Related online version (cited on 14 February 2008): http://arXiv.org/abs/hep-th/9911230. 9.2

[4] Ashtekar, A., and Magnon, A., "Asymptotically anti-de Sitter space-times", Class. Quantum Grav., 1, L39-L44, (1984). 9.2, 9.3

[5] Ashtekar, A., Pawlowski, T., and Van Den Broeck, C., "Mechanics of higher-dimensional black holes in asymptotically anti-de Sitter space-times", Class. Quantum Grav., 24, 625, (2007). Related online version (cited on 14 February 2008):

http://arXiv.org/abs/gr-qc/0611049. 8.9

[6] Astefanesei, D., and Radu, E., "Quasilocal formalism and black ring thermodynamics", Phys. Rev. D, 73, 044014, (2006). Related online version (cited on 14 February 2008):

http://arXiv.org/abs/hep-th/0509144. 8.7

[7] Azuma, T., and Koikawa, T., "Infinite number of stationary soliton solutions to fivedimensional vacuum Einstein equation", Prog. Theor. Phys., 116, 319, (2006). Related online version (cited on 14 February 2008):

http://arXiv.org/abs/hep-th/0512350. 5.2.2.2

[8] Barrabes, C., Frolov, V.P., and Lesigne, E., "Geometric inequalities and trapped surfaces in higher dimensional spacetimes", Phys. Rev. D, 69, 101501, (2004). Related online version (cited on 14 February 2008):

http://arXiv.org/abs/gr-qc/0402081. 8.9

[9] Behrndt, K., Cvetič, M., and Sabra, W.A., "Non-extreme black holes of five dimensional $N=2$ AdS supergravity", Nucl. Phys. B, 553, 317-332, (1999). Related online version (cited on 14 February 2008):

http://arXiv.org/abs/hep-th/9810227. 9.5

[10] Belinski, V., and Verdaguer, E., Gravitational Solitons, Cambridge Monographs on Mathematical Physics, (Cambridge University Press, Cambridge, U.K.; New York, U.S.A., 2001). $5.2 .2 .2,5.2 .2 .3$

[11] Belinsky, V.A., and Zakharov, V.E., "Integration of the Einstein Equations by the Inverse Scattering Problem Technique and the Calculation of the Exact Soliton Solutions", Sov. Phys. JETP, 48, 985, (1978). also in Zh. Eksp. Teor. Fiz., 75, (1953). 5.2.2.2

[12] Bena, I., and Warner, N.P., "One ring to rule them all ... and in the darkness bind them?", Adv. Theor. Math. Phys., 9, 667, (2005). Related online version (cited on 14 February 2008): http://arXiv.org/abs/hep-th/0408106. 7.3.2 
[13] Berman, D.S., and Parikh, M.K., "Holography and rotating AdS black holes", Phys. Lett. $B, 463,168,(1999)$. Related online version (cited on 14 February 2008): http://arXiv.org/abs/hep-th/9907003. 9.3

[14] Bhattacharyya, S., Lahiri, S., Loganayagam, R., and Minwalla, S., "Large rotating AdS black holes from fluid mechanics", (2007). URL (cited on 14 February 2008): http://arXiv.org/abs/arXiv:0708.1770. 9.3

[15] Birmingham, D., "Topological black holes in anti-de Sitter space", Class. Quantum Grav., 16, 1197, (1999). Related online version (cited on 14 February 2008): http://arXiv.org/abs/hep-th/9808032. 11

[16] Bouchareb, A., Clement, G., Chen, C.M., Gal'tsov, D.V., Scherbluk, N.G., and Wolf, T., " $G_{2}$ generating technique for minimal $\mathrm{D}=5$ supergravity and black rings", Phys. Rev. D, $\mathbf{7 6}$, 104032, (2007). Related online version (cited on 14 February 2008): http://arXiv.org/abs/arXiv:0708.2361.7.4

[17] Breckenridge, J.C., Myers, R.C., Peet, A.W., and Vafa, C., "D-branes and spinning black holes", Phys. Lett. B, 391, 93, (1997). Related online version (cited on 14 February 2008): http://arXiv.org/abs/hep-th/9602065. 7.2.2

[18] Buchel, A., and Liu, J.T., "Gauged supergravity from type IIB string theory on $Y^{p, q}$ manifolds", Nucl. Phys. B, 771, 93, (2007). Related online version (cited on 14 February 2008): http://arXiv.org/abs/hep-th/0608002. 9.4

[19] Bunting, G.L., and Masood-ul Alam, A.K.M., "Nonexistence of multiple black holes is asymptotically Euclidean static vacuum space-times", Gen. Relativ. Gravit., 19, 147-154, (1987). 8.3

[20] Caldarelli, M.M., Cognola, G., and Klemm, D., "Thermodynamics of Kerr-Newman-AdS black holes and conformal field theories", Class. Quantum Grav., 17, 399, (2000). Related online version (cited on 14 February 2008):

http://arXiv.org/abs/hep-th/9908022. 13, 9.3, 9.6

[21] Caldarelli, M.M., and Klemm, D., "Supersymmetry of anti-de Sitter black holes", Nucl. Phys. $B, \mathbf{5 4 5}, 434,(1999)$. Related online version (cited on 14 February 2008): http://arXiv.org/abs/hep-th/9808097. 9.6

[22] Candlish, G.N., and Reall, H.S., "On the smoothness of static multi-black hole solutions of higher-dimensional Einstein-Maxwell theory", Class. Quantum Grav., 24, 6025, (2007). Related online version (cited on 14 February 2008):

http://arXiv.org/abs/arXiv:0707.4420. 7.5

[23] Cardoso, V., and Dias, Ó.J.C., "Small Kerr-anti-de Sitter black holes are unstable", Phys. Rev. D, 70, 084011, (2004). Related online version (cited on 14 February 2008): http://arXiv.org/abs/hep-th/0405006. 9.3

[24] Cardoso, V., Dias, Ó.J.C., and Lemos, J.P.S., "Gravitational radiation in D-dimensional spacetimes", Phys. Rev. D, 67, 064026, (2003). Related online version (cited on 14 February 2008):

http://arXiv.org/abs/hep-th/0212168. 8.8

[25] Cardoso, V., Dias, Ó.J.C., Lemos, J.P.S., and Yoshida, S., "The black hole bomb and superradiant instabilities", Phys. Rev. D, 70, 044039, (2004). Related online version (cited on 14 February 2008):

http://arXiv.org/abs/hep-th/0404096. 8.4 
[26] Cardoso, V., Siopsis, G., and Yoshida, S., "Scalar perturbations of higher dimensional rotating and ultra-spinning black holes", Phys. Rev. D, 71, 024019, (2005). Related online version (cited on 14 February 2008):

http://arXiv.org/abs/hep-th/0412138. 4.4

[27] Carter, B., "Hamilton-Jacobi and Schrodinger separable solutions of Einstein's equations", Commun. Math. Phys., 10, 280, (1968). 9.3

[28] Carter, B., "Essentials of classical brane dynamics", Int. J. Theor. Phys., 40, 2099, (2001). Related online version (cited on 14 February 2008):

http://arXiv.org/abs/gr-qc/0012036. 6.1

[29] Castejon-Amenedo, J., and Manko, V.S., "Superposition of the Kerr metric with the generalized Erez-Rosen solution", Phys. Rev. D, 41, 2018-2020, (1990). 5.2.2.4

[30] Cavaglià, M., "Black hole and brane production in $\mathrm{TeV}$ gravity: A review", Int. J. Mod. Phys. A, 18, 1843-1882, (2003). Related online version (cited on 14 February 2008):

http://arXiv.org/abs/hep-ph/0210296. 1, 2.1, 8.8

[31] Chamblin, A., Emparan, R., Johnson, C.V., and Myers, R.C., "Charged AdS black holes and catastrophic holography", Phys. Rev. D, 60, 064018, (1999). Related online version (cited on 14 February 2008):

http://arXiv.org/abs/hep-th/9902170. 9.4,9.5

[32] Chamblin, A., Emparan, R., Johnson, C.V., and Myers, R.C., "Holography, thermodynamics and fluctuations of charged AdS black holes", Phys. Rev. D, 60, 104026, (1999). Related online version (cited on 14 February 2008):

http://arXiv.org/abs/hep-th/9904197. 9.5

[33] Charmousis, C., "Higher order gravity theories and their black hole solutions", (2008). URL (cited on 15 July 2008):

http://arXiv.org/abs/0805.0568. 2.1

[34] Charmousis, C., and Gregory, R., "Axisymmetric metrics in arbitrary dimensions", Class. Quantum Grav., 21, 527, (2004). Related online version (cited on 14 February 2008): http://arXiv.org/abs/gr-qc/0306069. 1.2, 6

[35] Charmousis, C., Langlois, D., Steer, D., and Zegers, R., "Rotating spacetimes with a cosmological constant", J. High Energy Phys., 2007(02), 064, (2007). Related online version (cited on 14 February 2008):

http://arXiv.org/abs/gr-qc/0610091. 1.2, 6

[36] Chong, Z.W., Cvetič, M., Lü, H., and Pope, C.N., "Charged rotating black holes in fourdimensional gauged and ungauged supergravities", Nucl. Phys. B, 717, 246, (2005). Related online version (cited on 14 February 2008):

http://arXiv.org/abs/hep-th/0411045. 9.6

[37] Chong, Z.W., Cvetič, M., Lü, H., and Pope, C.N., "Five-dimensional gauged supergravity black holes with independent rotation parameters", Phys. Rev. D, 72, 041901, (2005). Related online version (cited on 14 February 2008):

http://arXiv.org/abs/hep-th/0505112. 9.6

[38] Chong, Z.W., Cvetič, M., Lü, H., and Pope, C.N., "General non-extremal rotating black holes in minimal five-dimensional gauged supergravity", Phys. Rev. Lett., 95, 161301, (2005). Related online version (cited on 14 February 2008):

http://arXiv.org/abs/hep-th/0506029. 9.6 
[39] Chong, Z.W., Cvetič, M., Lü, H., and Pope, C.N., "Non-extremal rotating black holes in fivedimensional gauged supergravity", Phys. Lett. B, 644, 192, (2007). Related online version (cited on 14 February 2008):

http://arXiv.org/abs/hep-th/0606213. 9.6

[40] Chong, Z.W.H., Cvetič, M., Lü, H., and Pope, C.N., "Non-extremal charged rotating black holes in seven-dimensional gauged supergravity", Phys. Lett. B, 626, 215, (2005). Related online version (cited on 14 February 2008):

http://arXiv.org/abs/hep-th/0412094. 9.6

[41] Choptuik, M.W., "Universality and scaling in gravitational collapse of a massless scalar field", Phys. Rev. Lett., 70, 9-12, (1993). 8.9

[42] Choptuik, M.W., Lehner, L., Olabarrieta, I., Petryk, R., Pretorius, F., and Villegas, H., "Towards the final fate of an unstable black string", Phys. Rev. D, 68, 044001, (2003). Related online version (cited on 14 February 2008):

http://arXiv.org/abs/gr-qc/0304085. 3.4

[43] Chow, D.D.K., "Equal charge black holes and seven dimensional gauged supergravity", (2007). URL (cited on 14 February 2008):

http://arXiv.org/abs/arXiv:0711.1975. 9.6

[44] Chruściel, P.T., "The classification of static vacuum space-times containing an asymptotically flat spacelike hypersurface with compact interior", Class. Quantum Grav., 16, 661, (1999). Related online version (cited on 14 February 2008):

http://arXiv.org/abs/gr-qc/9809088. 8.3

[45] Chruściel, P.T., Reall, H.S., and Tod, P., "On Israel-Wilson-Perjes black holes", Class. Quantum Grav., 23, 2519, (2006). Related online version (cited on 14 February 2008): http://arXiv.org/abs/gr-qc/0512116. 8.5

[46] Chruściel, P.T., Reall, H.S., and Tod, P., "On non-existence of static vacuum black holes with degenerate components of the event horizon", Class. Quantum Grav., 23, 549, (2006). Related online version (cited on 14 February 2008):

http://arXiv.org/abs/gr-qc/0512041. 8.3

[47] Chruściel, P.T., and Tod, P., "The classification of static electro-vacuum space-times containing an asymptotically flat spacelike hypersurface with compact interior", Commun. Math. Phys., 271, 577, (2007). Related online version (cited on 14 February 2008):

http://arXiv.org/abs/gr-qc/0512043. 8.3

[48] Chruściel, P.T., and Wald, R.M., "On the Topology of Stationary Black Holes", Class. Quantum Grav., 11, L147-L152, (1994). Related online version (cited on 14 February 2008): http://arXiv.org/abs/gr-qc/9410004. 8.2

[49] Chu, Y.Z., Goldberger, W.D., and Rothstein, I.Z., "Asymptotics of d-dimensional KaluzaKlein black holes: Beyond the newtonian approximation", J. High Energy Phys., 2006(03), 013, (2006). Related online version (cited on 14 February 2008):

http://arXiv.org/abs/hep-th/0602016. 7

[50] Coley, A., Milson, R., Pravda, V., and Pravdova, A., "Classification of the Weyl tensor in higher-dimensions", Class. Quantum Grav., 21, L35, (2004). Related online version (cited on 14 February 2008):

http://arXiv.org/abs/gr-qc/0401008. 8.6 
[51] Coley, A.A., "Classification of the Weyl Tensor in Higher Dimensions and Applications", (2007). URL (cited on 14 February 2008): http://arXiv.org/abs/arXiv:0710.1598. 1.2, 8.6

[52] Copsey, K., and Horowitz, G.T., "The role of dipole charges in black hole thermodynamics", Phys. Rev. D, 73, 024015, (2006). Related online version (cited on 14 February 2008): http://arXiv.org/abs/hep-th/0505278. 8.7

[53] Cosgrove, C.M., "Relationships between the group-theoretic and soliton-theoretic techniques for generating stationary axisymmetric gravitational solutions", J. Math. Phys., 21, 2417, (1980). 5.2.2.4

[54] Cosgrove, C.M., "Relationship between the inverse scattering techniques of BelinskiiZakharov and Hauser-Ernst in general relativity", J. Math. Phys., 23, 615, (1982). 5.2.2.4

[55] Cvetič, M., Duff, M.J., Hoxha, P., Liu, J.T., Lü, H., Lu, J.X., Martinez-Acosta, R., Pope, C.N., Sati, H., and Tran, T.A., "Embedding AdS black holes in ten and eleven dimensions", Nucl. Phys. B, 558, 96-126, (1999). Related online version (cited on 14 February 2008): http://arXiv.org/abs/hep-th/9903214. 7.2.1, 9.4, 9.5

[56] Cvetič, M., and Gubser, S.S., "Phases of R-charged black holes, spinning branes and strongly coupled gauge theories", J. High Energy Phys., 1999(04), 024, (1999). Related online version (cited on 14 February 2008): http://arXiv.org/abs/hep-th/9902195. 9.5

[57] Cvetič, M., and Gubser, S.S., "Thermodynamic stability and phases of general spinning branes", J. High Energy Phys., 1999(07), 010, (1999). Related online version (cited on 14 February 2008): http://arXiv.org/abs/hep-th/9903132. 9.5

[58] Cvetič, M., and Hull, C.M., "Black holes and U-duality", Nucl. Phys. B, 480, 296, (1996). Related online version (cited on 14 February 2008): http://arXiv.org/abs/hep-th/9606193. 7.1, 7.2.1

[59] Cvetič, M., Lü, H., and Pope, C.N., "Gauged six-dimensional supergravity from massive type IIA", Phys. Rev. Lett., 83, 5226, (1999). Related online version (cited on 14 February 2008): http://arXiv.org/abs/hep-th/9906221. 9.4, 9.5

[60] Cvetič, M., Lü, H., and Pope, C.N., "Charged Kerr-de Sitter black holes in five dimensions", Phys. Lett. B, 598, 273, (2004). Related online version (cited on 14 February 2008): http://arXiv.org/abs/hep-th/0406196. 9.6

[61] Cvetič, M., Lü, H., and Pope, C.N., "Charged rotating black holes in five dimensional U(1)**3 gauged N = 2 supergravity", Phys. Rev. D, 70, 081502, (2004). Related online version (cited on 14 February 2008):

http://arXiv.org/abs/hep-th/0407058. 9.6

[62] Cvetič, M., Lü, H., Pope, C.N., Sadrzadeh, A., and Tran, T.A., "Consistent SO(6) reduction of type IIB supergravity on $S^{5 "}$, Nucl. Phys. B, 586, 275, (2000). Related online version (cited on 14 February 2008): http://arXiv.org/abs/hep-th/0003103. 9.4

[63] Cvetič, M., and Youm, D., "General Rotating Five Dimensional Black Holes of Toroidally Compactified Heterotic String", Nucl. Phys. B, 476, 118, (1996). Related online version (cited on 14 February 2008): http://arXiv.org/abs/hep-th/9603100. 7.2.1, 7.2.2 
[64] Cvetič, M., and Youm, D., "Near-BPS-Saturated Rotating Electrically Charged Black Holes as String States", Nucl. Phys. B, 477, 449, (1996). Related online version (cited on 14 February 2008):

http://arXiv.org/abs/hep-th/9605051. 7.2.1

[65] De Smet, P.J., "Black holes on cylinders are not algebraically special", Class. Quantum Grav., 19, 4877, (2002). Related online version (cited on 14 February 2008):

http://arXiv.org/abs/hep-th/0206106. 8.6

[66] De Smet, P.J., "The Petrov type of the five-dimensional Myers-Perry metric", Gen. Relativ. Gravit., 36, 1501, (2004). Related online version (cited on 14 February 2008): http://arXiv.org/abs/gr-qc/0312021. 8.6

[67] de Wit, B., and Nicolai, H., "The Consistency of the $\mathrm{S}^{* *} 7$ Truncation in D=11 Supergravity", Nucl. Phys. B, 281, 211, (1987). 9.4

[68] Dias, Ó.J.C., "Superradiant instability of large radius doubly spinning black rings", Phys. Rev. D, 73, 124035, (2006). Related online version (cited on 14 February 2008): http://arXiv.org/abs/hep-th/0602064. 5.4

[69] Duff, M.J., and Liu, J.T., "Anti-de Sitter black holes in gauged $N=8$ supergravity", Nucl. Phys. B, 554, 237-253, (1999). Related online version (cited on 14 February 2008): http://arXiv.org/abs/hep-th/9901149. 9.5

[70] Elvang, H., "A charged rotating black ring", Phys. Rev. D, 68, 124016, (2003). Related online version (cited on 14 February 2008): http://arXiv.org/abs/hep-th/0305247. 7.3.2

[71] Elvang, H., and Emparan, R., "Black rings, supertubes, and a stringy resolution of black hole non-uniqueness", J. High Energy Phys., 2003(11), 035, (2003). Related online version (cited on 14 February 2008): http://arXiv.org/abs/hep-th/0310008. 5.4, 6.1, 7.3.2, 7.4

[72] Elvang, H., Emparan, R., and Figueras, P., "Non-supersymmetric black rings as thermally excited supertubes", J. High Energy Phys., 2005(02), 031, (2005). Related online version (cited on 14 February 2008): http://arXiv.org/abs/hep-th/0412130. 7.3.2, 7.4

[73] Elvang, H., Emparan, R., and Figueras, P., "Phases of Five-Dimensional Black Holes", J. High Energy Phys., 2007(05), 056, (2007). Related online version (cited on 14 February 2008):

http://arXiv.org/abs/hep-th/0702111. 5.3, 5.3, 8.7, 8.7

[74] Elvang, H., Emparan, R., Mateos, D., and Reall, H.S., "A supersymmetric black ring", Phys. Rev. Lett., 93, 211302, (2004). Related online version (cited on 14 February 2008): http://arXiv.org/abs/hep-th/0407065. 7.3.2, 8.5

[75] Elvang, H., Emparan, R., Mateos, D., and Reall, H.S., "Supersymmetric black rings and three-charge supertubes", Phys. Rev. D, 71, 024033, (2005). Related online version (cited on 14 February 2008):

http://arXiv.org/abs/hep-th/0408120. 7.3.2

[76] Elvang, H., Emparan, R., and Virmani, A., "Dynamics and stability of black rings", J. High Energy Phys., 2006(12), 074, (2006). Related online version (cited on 14 February 2008): http://arXiv.org/abs/hep-th/0608076. 5.1.1, 5.1.1, 5.4, 6, 8.8 
[77] Elvang, H., and Figueras, P., "Black Saturn", J. High Energy Phys., 2007(05), 050, (2007). Related online version (cited on 14 February 2008): http://arXiv .org/abs/hep-th/0701035. 5.2.2.3, 5.3, 5.4

[78] Elvang, H., and Rodriguez, M.J., "Bicycling Black Rings", (2007). URL (cited on 14 February 2008):

http://arXiv.org/abs/arXiv:0712.2425. 5.1.2, 5.1.2, 5.3

[79] Emparan, R., "Rotating circular strings, and infinite non-uniqueness of black rings", J. High Energy Phys., 2004(03), 064, (2004). Related online version (cited on 14 February 2008): http://arXiv.org/abs/hep-th/0402149. 5.1.1, 5.1.1, 7.3.1, 7.3.2, 7.4, 8.7

[80] Emparan, R., Harmark, T., Niarchos, V., Obers, N.A., and Rodríguez, M.J., "The Phase Structure of Higher-Dimensional Black Rings and Black Holes", J. High Energy Phys., 2007(10), 110, (2007). Related online version (cited on 14 February 2008): http://arXiv.org/abs/arXiv:0708.2181. 3.1, 5.1.1, 6, 6.1, 6.1, 6.2, 11, 6.2, 12, 8.4

[81] Emparan, R., and Myers, R.C., "Instability of ultra-spinning black holes", J. High Energy Phys., 2003(09), 025, (2003). Related online version (cited on 14 February 2008): http://arXiv.org/abs/hep-th/0308056. 1.1, 4.1, 4.2.1, 4.4, 6, 6.2, 8.4

[82] Emparan, R., and Reall, H.S., "Generalized Weyl solutions", Phys. Rev. D, 65, 084025, (2002). Related online version (cited on 14 February 2008): http://arXiv.org/abs/hep-th/0110258. 5.2, 5.2, 5.2.1, 5.2.1, 5.2.2.3, 5.2.2.3, 5.3

[83] Emparan, R., and Reall, H.S., "A rotating black ring in five dimensions", Phys. Rev. Lett., 88, 101101, (2002). Related online version (cited on 14 February 2008): http://arXiv.org/abs/hep-th/0110260. 1, 5.1.1, 5.4

[84] Emparan, R., and Reall, H.S., "Black rings", Class. Quantum Grav., 23, R169, (2006). Related online version (cited on 14 February 2008): http://arXiv.org/abs/hep-th/0608012. 2.1, 5.1.1, 5, 5.1.1, 7.3.2

[85] Evslin, J., and Krishnan, C., "The Black Di-Ring: An Inverse Scattering Construction", (2007). URL (cited on 14 February 2008): http://arXiv.org/abs/arXiv:0706.1231. 5.3

[86] Feinstein, A., and Vazquez-Mozo, M.A., "M-theory resolution of four-dimensional cosmological singularities", Nucl. Phys. B, 568, 405, (2000). Related online version (cited on 14 February 2008): http://arXiv.org/abs/hep-th/9906006. 6

[87] Figueras, P., "A black ring with a rotating 2-sphere", J. High Energy Phys., 2005(07), 039, (2005). Related online version (cited on 14 February 2008): http://arXiv.org/abs/hep-th/0505244. 5.1.2

[88] Frolov, V.P., "Hidden Symmetries of Higher-Dimensional Black Hole Spacetimes", (2007). URL (cited on 14 February 2008): http://arXiv.org/abs/arXiv:0712.4157. 4.3

[89] Frolov, V.P., and Goswami, R., "Surface geometry of 5D black holes and black rings", Phys. Rev. D, 75, 124001, (2007). Related online version (cited on 14 February 2008): http://arXiv.org/abs/gr-qc/0612033. 5.1.1 
[90] Frolov, V.P., Krtouš, P., and Kubizňák, D., "Separability of Hamilton-Jacobi and KleinGordon equations in general Kerr-NUT-AdS spacetimes", J. High Energy Phys., 2007(02), 005, (2007). Related online version (cited on 14 February 2008):

http://arXiv.org/abs/hep-th/0611245. 4.3, 9.3

[91] Frolov, V.P., and Kubizňák, D., "Hidden symmetries of higher-dimensional rotating black holes", Phys. Rev. Lett., 98, 011101, (2007). 4.3

[92] Frolov, V.P., Mazzitelli, F.D., and Paz, J.P., "Quantum effects near multidimensional black holes", Phys. Rev. D, 40, 948, (1989). 8.8

[93] Frolov, V.P., and Stojkovic, D., "Particle and light motion in a space-time of a fivedimensional rotating black hole", Phys. Rev. D, 68, 064011, (2003). Related online version (cited on 14 February 2008):

http://arXiv.org/abs/gr-qc/0301016. 4.3

[94] Frolov, V.P., and Stojkovic, D., "Quantum radiation from a 5-dimensional rotating black hole", Phys. Rev. D, 67, 084004, (2003). Related online version (cited on 14 February 2008): http://arXiv.org/abs/gr-qc/0211055. 4.3

[95] Galloway, G.J., "On the topology of the domain of outer communication", Class. Quantum Grav., 12, L99-L101, (1995). 8.2

[96] Galloway, G.J., "Rigidity of outer horizons and the topology of black holes", (2006). URL (cited on 14 February 2008):

http://arXiv.org/abs/gr-qc/0608118. 8.2

[97] Galloway, G.J., Schleich, K., Witt, D.M., and Woolgar, E., "Topological censorship and higher genus black holes", Phys. Rev. D, 60, 104039, (1999). Related online version (cited on 14 February 2008):

http://arXiv.org/abs/gr-qc/9902061. 12

[98] Galloway, G.J., and Schoen, R., "A generalization of Hawking's black hole topology theorem to higher dimensions", Commun. Math. Phys., 266, 571, (2006). Related online version (cited on 14 February 2008):

http://arXiv.org/abs/gr-qc/0509107. 1.2, 8.2

[99] Gauntlett, J.P., and Gutowski, J.B., "Concentric black rings", Phys. Rev. D, 71, 025013, (2005). Related online version (cited on 14 February 2008):

http://arXiv.org/abs/hep-th/0408010. 7.5

[100] Gauntlett, J.P., and Gutowski, J.B., "General concentric black rings", Phys. Rev. D, 71, 045002, (2005). Related online version (cited on 14 February 2008):

http://arXiv.org/abs/hep-th/0408122. 7.3.2, 7.5

[101] Gauntlett, J.P., Gutowski, J.B., Hull, C.M., Pakis, S., and Reall, H.S., "All supersymmetric solutions of minimal supergravity in five dimensions", Class. Quantum Grav., 20, 4587, (2003). Related online version (cited on 14 February 2008):

http://arXiv.org/abs/hep-th/0209114. 7.3.2, 8.5

[102] Gauntlett, J.P., Myers, R.C., and Townsend, P.K., "Black holes of D = 5 supergravity", Class. Quantum Grav., 16, 1, (1999). Related online version (cited on 14 February 2008):

http://arXiv.org/abs/hep-th/9810204. 7.2.2, 7.5 
[103] Gauntlett, J.P., Ó Colgáin, E., and Varela, O., "Properties of some conformal field theories with M-theory duals", J. High Energy Phys., 2007(02), 049, (2007). Related online version (cited on 14 February 2008):

http://arXiv.org/abs/hep-th/0611219. 9.4

[104] Gauntlett, J.P., and Varela, O., "Consistent Kaluza-Klein Reductions for General Supersymmetric AdS Solutions", (2007). URL (cited on 14 February 2008):

http://arXiv.org/abs/arXiv:0707.2315. 9.4

[105] Gibbons, G., and Hartnoll, S.A., "A gravitational instability in higher dimensions", Phys. Rev. D, 66, 064024, (2002). Related online version (cited on 14 February 2008):

http://arXiv.org/abs/hep-th/0206202. 3.3

[106] Gibbons, G.W., and Herdeiro, C.A.R., "Supersymmetric rotating black holes and causality violation", Class. Quantum Grav., 16, 3619, (1999). Related online version (cited on 14 February 2008):

http://arXiv.org/abs/hep-th/9906098. 7.2 .2

[107] Gibbons, G.W., and Hull, C.M., "A Bogomolny bound for general relativity and solitons in $N=2$ supergravity", Phys. Lett. B, 109, 190-194, (1982). 8.5

[108] Gibbons, G.W., Hull, C.M., and Warner, N.P., "The stability of gauged supergravity", Nucl. Phys. B, 218, 173-190, (1983). 9.3

[109] Gibbons, G.W., Ida, D., and Shiromizu, T., "Uniqueness and non-uniqueness of static black holes in higher dimensions", Phys. Rev. Lett., 89, 041101, (2002). Related online version (cited on 14 February 2008):

http://arXiv.org/abs/hep-th/0206049. 8.3

[110] Gibbons, G.W., Ida, D., and Shiromizu, T., "Uniqueness and non-uniqueness of static vacuum black holes in higher dimensions", Prog. Theor. Phys. Suppl., 148, 284, (2003). Related online version (cited on 14 February 2008):

http://arXiv.org/abs/gr-qc/0203004. 8.3

[111] Gibbons, G.W., Lü, H., Page, D.N., and Pope, C.N., "Rotating black holes in higher dimensions with a cosmological constant", Phys. Rev. Lett., 93, 171102, (2004). Related online version (cited on 14 February 2008):

http://arXiv.org/abs/hep-th/0409155. 4.3, 9.3

[112] Gibbons, G.W., Lü, H., Page, D.N., and Pope, C.N., "The general Kerr-de Sitter metrics in all dimensions", J. Geom. Phys., 53, 49, (2005). Related online version (cited on 14 February 2008):

http://arXiv.org/abs/hep-th/0404008. 4.3, 9.3

[113] Gibbons, G.W., Perry, M.J., and Pope, C.N., "The first law of thermodynamics for Kerranti-de Sitter black holes", Class. Quantum Grav., 22, 1503-1526, (2005). Related online version (cited on 14 February 2008):

http://arXiv.org/abs/hep-th/0408217. 9.3, 9.3

[114] Giusto, S., and Saxena, A., "Stationary axisymmetric solutions of five dimensional gravity", Class. Quantum Grav., 24, 4269, (2007). Related online version (cited on 14 February 2008): http://arXiv.org/abs/0705.4484. 5.2.2.4 
[115] Gorbonos, D., and Kol, B., "A dialogue of multipoles: Matched asymptotic expansion for caged black holes", J. High Energy Phys., 2004(06), 053, (2004). Related online version (cited on 14 February 2008):

http://arXiv.org/abs/hep-th/0406002. 6.1

[116] Gorbonos, D., and Kol, B., "Matched asymptotic expansion for caged black holes: Regularization of the post-Newtonian order", Class. Quantum Grav., 22, 3935, (2005). Related online version (cited on 14 February 2008):

http://arXiv.org/abs/hep-th/0505009. 6.1

[117] Gregory, R., and Laflamme, R., "Hypercylindrical black holes", Phys. Rev. D, 37, 305, (1988). 3.4, 6.2

[118] Gregory, R., and Laflamme, R., "Black strings and p-branes are unstable", Phys. Rev. Lett., 70, 2837, (1993). Related online version (cited on 14 February 2008):

http://arXiv.org/abs/hep-th/9301052. 1, 1.1, 3.4

[119] Gregory, R., and Laflamme, R., "The Instability of charged black strings and p-branes", Nucl. Phys. B, 428, 399, (1994). Related online version (cited on 14 February 2008):

http://arXiv.org/abs/arXiv:hep-th/9404071. 3.4

[120] Gubser, S.S., "On non-uniform black branes", Class. Quantum Grav., 19, 4825, (2002). Related online version (cited on 14 February 2008):

http://arXiv.org/abs/hep-th/0110193. 3.4, 6.2

[121] Gubser, S.S., and Mitra, I., "Instability of charged black holes in anti-de Sitter space", (2000). URL (cited on 14 February 2008):

http://arXiv.org/abs/hep-th/0009126. 9.5

[122] Gubser, S.S., and Mitra, I., "The evolution of unstable black holes in anti-de Sitter space", J. High Energy Phys., 2001(08), 018, (2001). Related online version (cited on 14 February 2008):

http://arXiv.org/abs/hep-th/0011127. 9.5

[123] Gutowski, J.B., "Uniqueness of five-dimensional supersymmetric black holes", J. High Energy Phys., 2004(08), 049, (2004). Related online version (cited on 14 February 2008):

http://arXiv.org/abs/hep-th/0404079. 8.5

[124] Gutowski, J.B., and Reall, H.S., "General supersymmetric $A d S_{5}$ black holes", J. High Energy Phys., 2004(04), 048, (2004). Related online version (cited on 14 February 2008):

http://arXiv.org/abs/hep-th/0401129. 9.6

[125] Gutowski, J.B., and Reall, H.S., "Supersymmetric $A d S_{5}$ black holes", J. High Energy Phys., 2004(02), 006, (2004). Related online version (cited on 14 February 2008):

http://arXiv.org/abs/hep-th/0401042. 9.6

[126] Harmark, T., "Small black holes on cylinders", Phys. Rev. D, 69, 104015, (2004). Related online version (cited on 14 February 2008):

http://arXiv.org/abs/hep-th/0310259. 6.1

[127] Harmark, T., "Stationary and axisymmetric solutions of higher-dimensional general relativity", Phys. Rev. D, 70, 124002, (2004). Related online version (cited on 14 February 2008): http://arXiv.org/abs/hep-th/0408141. 5.2, 5.2.2.1, 5.2.2.1 
[128] Harmark, T., Niarchos, V., and Obers, N.A., "Instabilities of black strings and branes", Class. Quantum Grav., 24, R1, (2007). Related online version (cited on 14 February 2008): http://arXiv .org/abs/hep-th/0701022. 3.4, 9.5

[129] Harmark, T., and Obers, N.A., "Phases of Kaluza-Klein black holes: A brief review", (2005). URL (cited on 14 February 2008): http://arXiv.org/abs/hep-th/0503020. 2.1

[130] Harmark, T., and Olesen, P., "On the structure of stationary and axisymmetric metrics", Phys. Rev. D, 72, 124017, (2005). Related online version (cited on 14 February 2008): http://arXiv.org/abs/hep-th/0508208. 5.2.2.1

[131] Hartle, J.B., and Hawking, S.W., "Solutions of the Einstein-Maxwell equations with many black holes", Commun. Math. Phys., 26, 87-101, (1972). 7.5, 8.5

[132] Hawking, S.W., and Ellis, G.F.R., The Large Scale Structure of Space-Time, Cambridge Monographs on Mathematical Physics, (Cambridge University Press, Cambridge, U.K., 1973). 1.1, 8.2, 8.4

[133] Hawking, S.W., Hunter, C.J., and Taylor-Robinson, M.M., "Rotation and the AdS/CFT correspondence", Phys. Rev. D, 59, 064005, (1999). Related online version (cited on 14 February 2008): http://arXiv.org/abs/hep-th/9811056. 9.3, 9.3

[134] Hawking, S.W., and Page, D.N., "Thermodynamics of Black Holes in Anti-de Sitter Space", Commun. Math. Phys., 87, 577-588, (1983). 9.2

[135] Hawking, S.W., and Reall, H.S., "Charged and rotating AdS black holes and their CFT duals", Phys. Rev. D, 61, 024014, (2000). Related online version (cited on 14 February 2008): http://arXiv.org/abs/hep-th/9908109. 9.3

[136] Helfgott, C., Oz, Y., and Yanay, Y., "On the topology of black hole event horizons in higher dimensions", J. High Energy Phys., 2006(02), 025, (2006). Related online version (cited on 14 February 2008): http://arXiv.org/abs/hep-th/0509013. 8.2

[137] Hoenselaers, C., Kinnersley, W., and Xanthopoulos, B.C., "Symmetries of the stationary Einstein-Maxwell equations. VI. Transformations which generate asymptotically flat spacetimes with arbitrary multipole moments", J. Math. Phys., 20, 2530, (1979). 5.2.2.4

[138] Hollands, S., Ishibashi, A., and Wald, R.M., "A higher dimensional stationary rotating black hole must be axisymmetric", Commun. Math. Phys., 271, 699, (2007). Related online version (cited on 14 February 2008):

http://arXiv.org/abs/gr-qc/0605106. 1.1, 8.4

[139] Hollands, S., and Yazadjiev, S., "Uniqueness theorem for 5-dimensional black holes with two axial Killing fields", (2007). URL (cited on 14 February 2008):

http://arXiv.org/abs/arXiv:0707.2775. 5.2, 8.4

[140] Hong, K., and Teo, E., "A new form of the C-metric", Class. Quantum Grav., 20, 3269, (2003). Related online version (cited on 14 February 2008): http://arXiv.org/abs/gr-qc/0305089. 5 
[141] Horowitz, G.T., and Hubeny, V.E., "Note on small black holes in $A d S_{p} \times S^{q}$ ", J. High Energy Phys., 0006, 031, (2000). Related online version (cited on 14 February 2008):

http://arXiv.org/abs/hep-th/0005288. 9.2

[142] Horowitz, G.T., and Maeda, K., "Fate of the black string instability", Phys. Rev. Lett., 87, 131301, (2001). Related online version (cited on 14 February 2008):

http://arXiv.org/abs/hep-th/0105111. 3

[143] Hoskisson, J., "Particle motion in the rotating black ring metric", (2007). URL (cited on 14 February 2008):

http://arXiv.org/abs/arXiv:0705.0117. 5.1.1, 5.4

[144] Hovdebo, J.L., and Myers, R.C., "Black rings, boosted strings and Gregory-Laflamme", Phys. Rev. D, 73, 084013, (2006). Related online version (cited on 14 February 2008): http://arXiv.org/abs/hep-th/0601079. 5.4, 6

[145] Ida, D., and Nakao, K.-I., "Isoperimetric inequality for higher-dimensional black holes", Phys. Rev. D, 66, 064026, (2002). Related online version (cited on 14 February 2008): http://arXiv.org/abs/gr-qc/0204082. 8.9

[146] Ida, D., and Uchida, Y., "Stationary Einstein-Maxwell fields in arbitrary dimensions", Phys. Rev. D, 68, 104014, (2003). Related online version (cited on 14 February 2008): http://arXiv.org/abs/gr-qc/0307095. 7.4

[147] Ida, D., Uchida, Y., and Morisawa, Y., "The scalar perturbation of the higher-dimensional rotating black holes", Phys. Rev. D, 67, 084019, (2003). Related online version (cited on 14 February 2008): http://arXiv.org/abs/gr-qc/0212035. 4.4

[148] Iguchi, H., and Mishima, T., "Solitonic generation of five-dimensional black ring solution", Phys. Rev. D, 73, 121501, (2006). Related online version (cited on 14 February 2008): http://arXiv .org/abs/hep-th/0604050. 5.2.2.3, 5.2.2.4

[149] Iguchi, H., and Mishima, T., "Solitonic generation of vacuum solutions in five-dimensional general relativity", Phys. Rev. D, 74, 024029, (2006). Related online version (cited on 14 February 2008):

http://arXiv.org/abs/hep-th/0605090. 5.2.2.4

[150] Iguchi, H., and Mishima, T., "Black di-ring and infinite nonuniqueness", Phys. Rev. D, 75, 064018, (2007). Related online version (cited on 14 February 2008):

http://arXiv.org/abs/hep-th/0701043. 5.3

[151] Ishibashi, A., and Kodama, H., "Stability of higher-dimensional Schwarzschild black holes", Prog. Theor. Phys., 110, 901, (2003). Related online version (cited on 14 February 2008): http://arXiv.org/abs/hep-th/0305185. 3.3, 8.4, 9.2

[152] Izumi, K., "Orthogonal black di-ring solution", (2007). URL (cited on 14 February 2008): http://arXiv.org/abs/arXiv:0712.0902. 5.3

[153] Jacobson, T., and Venkataramani, S., "Topology Of Event Horizons And Topological Censorship", Class. Quantum Grav., 12, 1055, (1995). Related online version (cited on 14 February 2008): http://arXiv.org/abs/gr-qc/9410023. 8.2 
[154] Jamsin, E., "A Note on Conserved Charges of Asymptotically Flat and Anti-de Sitter Spaces in Arbitrary Dimensions", (2007). URL (cited on 14 February 2008):

http://arXiv.org/abs/arXiv:0705.0484. 3.1

[155] Kanti, P., "Black holes in theories with large extra dimensions: A review", Int. J. Mod. Phys. A, 19, 4899, (2004). Related online version (cited on 14 February 2008):

http://arXiv.org/abs/hep-ph/0402168. 1, 2.1, 8.8

[156] Karasik, D., Sahabandu, C., Suranyi, P., and Wijewardhana, L.C.R., "Analytic approximation to 5 dimensional black holes with one compact dimension", Phys. Rev. D, 71, 024024, (2005). Related online version (cited on 14 February 2008):

http://arXiv.org/abs/hep-th/0410078. 6.1

[157] Kay, B.S., and Wald, R.M., "Theorems on the Uniqueness and Thermal Properties of Stationary, Nonsingular, Quasifree States on Space-Times with a Bifurcate Killing Horizon", Phys. Rep., 207, 49-136, (1991). 5.3

[158] Kimura, M., Murata, K., Ishihara, H., and Soda, J., "On the Stability of Squashed KaluzaKlein Black Holes", (2007). URL (cited on 14 February 2008):

http://arXiv.org/abs/arXiv:0712.4202. 4.4

[159] Kinnersley, W., "Type D vacuum metrics", J. Math. Phys., 10, 1195, (1969). 8.6

[160] Kinney, J., Maldacena, J.M., Minwalla, S., and Raju, S., "An index for 4 dimensional super conformal theories", Commun. Math. Phys., 275, 209, (2007). Related online version (cited on 14 February 2008):

http://arXiv.org/abs/hep-th/0510251. 9.6

[161] Kleihaus, B., Kunz, J., and Navarro-Lerida, F., "Rotating Black Holes in Higher Dimensions", (2007). URL (cited on 14 February 2008):

http://arXiv.org/abs/arXiv:0710.2291. 2.1

[162] Kodama, H., "Perturbative uniqueness of black holes near the static limit in arbitrary dimensions", Prog. Theor. Phys., 112, 249, (2004). Related online version (cited on 14 February 2008):

http://arXiv.org/abs/hep-th/0403239. 8.4, 9.3

[163] Kodama, H., and Ishibashi, A., "A master equation for gravitational perturbations of maximally symmetric black holes in higher dimensions", Prog. Theor. Phys., 110, 701, (2003). Related online version (cited on 14 February 2008):

http://arXiv.org/abs/hep-th/0305147. 3.3, 9.2

[164] Kodama, H., and Ishibashi, A., "Master equations for perturbations of generalized static black holes with charge in higher dimensions", Prog. Theor. Phys., 111, 29, (2004). Related online version (cited on 14 February 2008):

http://arXiv.org/abs/hep-th/0308128. 7.2.1, 9.5, 13

[165] Koikawa, T., "Infinite number of soliton solutions to 5-dimensional vacuum Einstein equation", Prog. Theor. Phys., 114, 793, (2005). Related online version (cited on 14 February 2008):

http://arXiv.org/abs/hep-th/0501248. 5.2.1, 5.2.2.2

[166] Kol, B., "Speculative generalization of black hole uniqueness to higher dimensions", (2002). URL (cited on 14 February 2008):

http://arXiv.org/abs/arXiv:hep-th/0208056. 8.4 
[167] Kol, B., "The phase transition between caged black holes and black strings: A review", Phys. Rep., 422, 119, (2006). Related online version (cited on 14 February 2008):

http://arXiv.org/abs/hep-th/0411240. 3.4

[168] Kol, B., and Smolkin, M., "Classical Effective Field Theory and Caged Black Holes", (2007). URL (cited on 14 February 2008):

http://arXiv.org/abs/arXiv:0712.2822. 7

[169] Konoplya, R.A., and Zhidenko, A., "Stability of multidimensional black holes: Complete numerical analysis", Nucl. Phys. B, 777, 182, (2007). Related online version (cited on 14 February 2008):

http://arXiv.org/abs/hep-th/0703231. 7.2.1

[170] Korzynski, M., Lewandowski, J., and Pawlowski, T., "Mechanics of multidimensional isolated horizons", Class. Quantum Grav., 22, 2001, (2005). Related online version (cited on 14 February 2008):

http://arXiv.org/abs/gr-qc/0412108. 8.9

[171] Kostelecky, V.A., and Perry, M.J., "Solitonic Black Holes in Gauged N=2 Supergravity", Phys. Lett. B, 371, 191, (1996). Related online version (cited on 14 February 2008):

http://arXiv.org/abs/hep-th/9512222. 9.6

[172] Kottler, F., "The physical basis of Einstein's theory of gravitation", Ann. Phys. (Leipzig), 56, 401, (1918). 9.2

[173] Krtouš, P., Kubizňák, D., Page, D.N., and Vasudevan, M., "Constants of geodesic motion in higher-dimensional black-hole spacetimes", Phys. Rev. D, 76, 084034, (2007). Related online version (cited on 14 February 2008):

http://arXiv.org/abs/arXiv:0707.0001. 4.3, 9.3

[174] Kubizňák, D., and Frolov, V.P., "Hidden symmetry of higher dimensional Kerr-NUT-AdS spacetimes", Class. Quantum Grav., 24, F1-F6, (2007). Related online version (cited on 14 February 2008):

http://arXiv.org/abs/gr-qc/0610144. 4.3

[175] Kunduri, H.K., and Lucietti, J., "Electrically charged dilatonic black rings", Phys. Lett. B, 609, 143, (2005). Related online version (cited on 14 February 2008):

http://arXiv.org/abs/hep-th/0412153. 7.4

[176] Kunduri, H.K., and Lucietti, J., "Near-horizon geometries of supersymmetric $A d S_{5}$ black holes", (2007). URL (cited on 14 February 2008):

http://arXiv.org/abs/arXiv:0708.3695. 9.6

[177] Kunduri, H.K., Lucietti, J., and Reall, H.S., "Gravitational perturbations of higher dimensional rotating black holes: Tensor Perturbations", Phys. Rev. D, 74, 084021, (2006). Related online version (cited on 14 February 2008):

http://arXiv.org/abs/hep-th/0606076. 4.4, 9.3

[178] Kunduri, H.K., Lucietti, J., and Reall, H.S., "Supersymmetric multi-charge $A d S_{5}$ black holes", J. High Energy Phys., 2006(04), 036, (2006). Related online version (cited on 14 February 2008):

http://arXiv.org/abs/hep-th/0601156. 9.6 
[179] Kunduri, H.K., Lucietti, J., and Reall, H.S., "Do supersymmetric anti-de Sitter black rings exist?", J. High Energy Phys., 2007(02), 026, (2007). Related online version (cited on 14 February 2008):

http://arXiv.org/abs/hep-th/0611351. 9.6

[180] Lahiri, S., and Minwalla, S., "Plasmarings as dual black rings", (2007). URL (cited on 14 February 2008):

http: //arXiv.org/abs/arXiv:0705.3404. 6.2

[181] Larsen, F., "Entropy of thermally excited black rings", J. High Energy Phys., 2005(10), 100, (2005). Related online version (cited on 14 February 2008):

http://arXiv.org/abs/hep-th/0505152. 7.3.2

[182] Lewandowski, J., and Pawlowski, T., "Quasi-local rotating black holes in higher dimension: Geometry", Class. Quantum Grav., 22, 1573, (2005). Related online version (cited on 14 February 2008):

http://arXiv.org/abs/gr-qc/0410146. 8.9

[183] Liko, T., and Booth, I., "Supersymmetric isolated horizons", (2007). URL (cited on 14 February 2008):

http://arXiv.org/abs/arXiv:0712.3308. 8.9

[184] Llatas, P.M., "Electrically Charged Black-holes for the Heterotic String Compactified on a (10-D)-torus", Phys. Lett. B, 397, 63, (1997). Related online version (cited on 14 February 2008): http://arXiv.org/abs/hep-th/9605058. 7.2.1

[185] London, L.A.J., "Arbitrary dimensional cosmological multi-black holes", Nucl. Phys. B, 434, 709-735, (1995). 9.5

[186] Maartens, R., "Brane-World Gravity", Living Rev. Relativity, 7, lrr-2004-7, (2004). URL (cited on 18 February 2008):

http://www. livingreviews .org/lrr-2004-7. 2.1

[187] Maldacena, J.M., "The large N limit of superconformal field theories and supergravity", Adv. Theor. Math. Phys., 2, 231-252, (1998). Related online version (cited on 4 July 2008): http://arXiv.org/abs/hep-th/9711200. 1

[188] Maldacena, J.M., and Strominger, A., "Black hole greybody factors and D-brane spectroscopy", Phys. Rev. D, 55, 861-870, (1997). Related online version (cited on 14 February 2008):

http://arXiv.org/abs/hep-th/9609026. 8.8

[189] Manko, V.S., and Sibgatullin, N.R., "Construction of exact solutions of the Einstein-Maxwell equations corresponding to a given behaviour of the Ernst potentials on the symmetry axis", Class. Quantum Grav., 10, 1383, (1993). 5.2.2.4

[190] Marolf, D., "On the fate of black string instabilities: An observation", Phys. Rev. D, 71, 127504, (2005). Related online version (cited on 14 February 2008): http://arXiv.org/abs/hep-th/0504045. 3

[191] Mei, J., and Pope, C.N., "New Rotating Non-Extremal Black Holes in D=5 Maximal Gauged Supergravity", (2007). URL (cited on 14 February 2008): http://arXiv.org/abs/arXiv:0709.0559. 9.6 
[192] Mishima, T., and Iguchi, H., "New axisymmetric stationary solutions of five-dimensional vacuum Einstein equations with asymptotic flatness", Phys. Rev. D, 73, 044030, (2006). Related online version (cited on 14 February 2008):

http://arXiv.org/abs/hep-th/0504018. 5.1.2, 5.2.2.4

[193] Miyamoto, U., and Murata, K., "On Hawking radiation from black rings", (2007). URL (cited on 14 February 2008):

http://arXiv.org/abs/arXiv:0705.3150. 8.8

[194] Morisawa, Y., and Ida, D., "A boundary value problem for the five-dimensional stationary rotating black holes", Phys. Rev. D, 69, 124005, (2004). Related online version (cited on 14 February 2008):

http://arXiv.org/abs/gr-qc/0401100. 8.4

[195] Morisawa, Y., and Ida, D., "Scalar field perturbation on six-dimensional ultra-spinning black holes", Phys. Rev. D, 71, 044022, (2005). Related online version (cited on 14 February 2008): http://arXiv.org/abs/gr-qc/0412070. 4.4

[196] Morisawa, Y., Tomizawa, S., and Yasui, Y., "Boundary Value Problem for Black Rings", (2007). URL (cited on 14 February 2008):

http://arXiv.org/abs/arXiv:0710.4600. 5.1.2, 5.1.2

[197] Murata, K., and Soda, J., "A Note on Separability of Field Equations in Myers-Perry Spacetimes", Class. Quantum Grav., 25, 035006, (2008). Related online version (cited on 14 February 2008):

http://arXiv.org/abs/arXiv:0710.0221. 4.4

[198] Myers, R.C., "Higher dimensional black holes in compactified space-times", Phys. Rev. D, 35, 455, (1987). 7.5

[199] Myers, R.C., "Black holes in higher curvature gravity", (1998). URL (cited on 14 February 2008):

http://arXiv.org/abs/gr-qc/9811042. 2.1

[200] Myers, R.C., and Perry, M.J., "Black Holes In Higher Dimensional Space-Times", Ann. Phys. (N.Y.), 172, 304, (1986). 1.1, 4, 4, 4.2, 4.2.1, 4.2.2

[201] Nastase, H., Vaman, D., and van Nieuwenhuizen, P., "Consistent nonlinear K K reduction of 11d supergravity on $A d S_{7} \times S_{4}$ ", Phys. Lett. B, 469, 96, (1999). Related online version (cited on 14 February 2008):

http://arXiv.org/abs/hep-th/9905075. 9.4

[202] Nastase, H., Vaman, D., and van Nieuwenhuizen, P., "Consistency of the $A d S_{7} \times S_{4}$ ", Nucl. Phys. B, 581, 179, (2000). Related online version (cited on 14 February 2008):

http://arXiv.org/abs/hep-th/9911238. 9.4

[203] Neugebauer, G., "Bäcklund transformations of axially symmetric stationary gravitational fields", J. Phys. A, 12, L67, (1979). 5.2.2.4

[204] Nomura, H., Yoshida, S., Tanabe, M., and Maeda, K.-I., "The fate of a five-dimensional rotating black hole via Hawking radiation", Prog. Theor. Phys., 114, 707, (2005). Related online version (cited on 14 February 2008):

http://arXiv.org/abs/hep-th/0502179. 8.8 
[205] Nozawa, M., and Maeda, K.-I., "Energy extraction from higher dimensional black holes and black rings", Phys. Rev. D, 71, 084028, (2005). Related online version (cited on 14 February 2008):

http://arXiv.org/abs/hep-th/0502166. 5.1.1

[206] Ooguri, H., "Spectrum of Hawking Radiation and Huygens' Principle", Phys. Rev. D, 33, 3573, (1986). 8.8

[207] Page, D.N., Kubizñák, D., Vasudevan, M., and Krtouš, P., "Complete Integrability of Geodesic Motion in General Higher-Dimensional Rotating Black-Hole Spacetimes", Phys. Rev. Lett., 98, 061102, (2007). Related online version (cited on 14 February 2008):

http://arXiv.org/abs/hep-th/0611083. 4.3,9.3

[208] Penrose, R., "Naked singularities", Ann. N.Y. Acad. Sci., 224, 125-134, (1973). 8.9

[209] Podolsky, J., and Ortaggio, M., "Robinson-Trautman spacetimes in higher dimensions", Class. Quantum Grav., 23, 5785, (2006). Related online version (cited on 14 February 2008): http://arXiv.org/abs/gr-qc/0605136. 8.6

[210] Polchinski, J., String Theory. Vol. 2: Superstring theory and beyond, Cambridge Monographs on Mathematical Physics, (Cambridge University Press, Cambridge, U.K.; New York, U.S.A., 1998). 7.1

[211] Pomeransky, A.A., "Complete integrability of higher-dimensional Einstein equations with additional symmetry, and rotating black holes", Phys. Rev. D, 73, 044004, (2006). Related online version (cited on 14 February 2008):

http://arXiv.org/abs/hep-th/0507250. 5.2.2.2, 5.2.2.3, 5.2.2.3

[212] Pomeransky, A.A., and Sen'kov, R.A., "Black ring with two angular momenta", (2006). URL (cited on 14 February 2008):

http://arXiv.org/abs/hep-th/0612005. 2.1, 5.1.2, 5.1.2, 5.1.2, 5.2.2.2, 5.2.2.3

[213] Pravda, V., and Pravdova, A., "WANDs of the black ring", Gen. Relativ. Gravit., 37, 1277, (2005). Related online version (cited on 14 February 2008):

http://arXiv.org/abs/gr-qc/0501003. 8.6

[214] Pravda, V., Pravdova, A., Coley, A., and Milson, R., "Bianchi identities in higher dimensions", Class. Quantum Grav., 21, 1691, (2004). Related online version (cited on 14 February 2008):

http://arXiv.org/abs/gr-qc/0401013. Erratum Class. Quantum Grav., 24, (2007. 8.6

[215] Pravda, V., Pravdova, A., and Ortaggio, M., "Type D Einstein spacetimes in higher dimensions", (2007). URL (cited on 14 February 2008):

http: //arXiv.org/abs/arXiv:0704.0435. 8.6

[216] Reall, H.S., "Higher dimensional black holes and supersymmetry", Phys. Rev. D, 68, 024024, (2003). Related online version (cited on 14 February 2008):

http://arXiv.org/abs/hep-th/0211290. Erratum Phys. Rev. D, 70,089902 (2004). 8.4, 8.5

[217] Reall, H.S., "Counting the microstates of a vacuum black ring", (2007). URL (cited on 14 February 2008):

http://arXiv.org/abs/arXiv:0712.3226. 1 
[218] Rogatko, M., "Uniqueness theorem of static degenerate and non-degenerate charged black holes in higher dimensions", Phys. Rev. D, 67, 084025, (2003). Related online version (cited on 14 February 2008):

http://arXiv.org/abs/hep-th/0302091. 8.3

[219] Rogatko, M., "Staticity theorem for higher dimensional generalized Einstein-Maxwell system", Phys. Rev. D, 71, 024031, (2005). Related online version (cited on 14 February 2008): http://arXiv.org/abs/hep-th/0501216. 8.4

[220] Rogatko, M., "Classification of static charged black holes in higher dimensions", Phys. Rev. D, 73, 124027, (2006). Related online version (cited on 14 February 2008): http://arXiv.org/abs/hep-th/0606116. 8.3

[221] Romans, L.J., "Supersymmetric, cold and lukewarm black holes in cosmological EinsteinMaxwell theory", Nucl. Phys. B, 383, 395, (1992). Related online version (cited on 14 February 2008): http://arXiv.org/abs/hep-th/9203018. 9.5

[222] Schwartz, F., "Existence of outermost apparent horizons with product of spheres topology", (2007). URL (cited on 14 February 2008): http://arXiv.org/abs/arXiv:0704.2403. 8.9

[223] Senovilla, J.M.M., "Trapped surfaces, horizons, and exact solutions in higher dimensions", Class. Quantum Grav., 19, L113, (2002). Related online version (cited on 14 February 2008): http://arXiv.org/abs/hep-th/0204005. 8.9

[224] Senovilla, J.M.M., "A Reformulation of the Hoop Conjecture", (2007). URL (cited on 14 February 2008): http://arXiv.org/abs/arXiv:0709.0695. 8.9

[225] Shiromizu, T., and Tomizawa, S., "Comment on spatial infinity in higher dimensional spacetimes", Phys. Rev. D, 69, 104012, (2004). Related online version (cited on 14 February 2008): http://arXiv.org/abs/gr-qc/0401006. 8.4

[226] Sibgatullin, N.R., Oscillations and Waves in Strong Gravitational and Electromagnetic Fields, (Springer, Berlin, Germany; New York, U.S.A., 1991). English translation of 1984 edition. 5.2.2.4

[227] Sorkin, E., and Oren, Y., "On Choptuik's scaling in higher dimensions", Phys. Rev. D, 71, 124005, (2005). Related online version (cited on 14 February 2008):

http://arXiv.org/abs/hep-th/0502034. 8.9

[228] Stephani, H., Kramer, D., MacCallum, M., Hoenselaers, C., and Herlt, E., Exact Solutions of Einstein's Field Equations, Cambridge Monographs on Mathematical Physics, (Cambridge University Press, Cambridge, U.K.; New York, U.S.A., 2003), 2nd edition. 8.5

[229] Strominger, A., and Vafa, C., "Microscopic Origin of the Bekenstein-Hawking Entropy", Phys. Lett. B, 379, 99-104, (1996). Related online version (cited on 14 February 2008): http://arXiv .org/abs/hep-th/9601029. 1, 7.2.2

[230] Sudarsky, D., and Wald, R.M., "Extrema of mass, stationarity, and staticity, and solutions to the Einstein-Yang-Mills equations", Phys. Rev. D, 46, 1453-1474, (1992). 8.4

[231] Tan, H.S., and Teo, E., "Multi-black hole solutions in five dimensions", Phys. Rev. D, 68, 044021, (2003). Related online version (cited on 14 February 2008):

http://arXiv.org/abs/hep-th/0306044. 5.3 
[232] Tangherlini, F.R., "Schwarzschild field in $\mathrm{n}$ dimensions and the dimensionality of space problem", Nuovo Cimento, 27, 636, (1963). 3.2

[233] Teo, E., "Black diholes in five dimensions", Phys. Rev. D, 68, 084003, (2003). Related online version (cited on 14 February 2008): http://arXiv.org/abs/hep-th/0307188. 7.4

[234] Teukolsky, S.A., "Rotating black holes: Separable wave equations for gravitational and electromagnetic perturbations", Phys. Rev. Lett., 29, 1114-1118, (1972). 4.4

[235] Teukolsky, S.A., "Perturbations of a rotating black hole. I. Fundamental equations for gravitational electromagnetic, and neutrino field perturbations", Astrophys. J., 185, 635-648, (1973). 4.4

[236] Thorne, K.S., "Non-spherical gravitational collapse - A short review", in Klauder, J., ed., Magic without Magic: John Archibald Wheeler. A Collection of Essays in Honor of his Sixtieth Birthday, pp. 231-258, (W.H. Freeman, San Francisco, U.S.A., 1972). 8.9

[237] Tod, K.P., "All metrics admitting super-covariantly constant spinors", Phys. Lett. B, 121, 241-244, (1983). 8.5

[238] Tomizawa, S., Iguchi, H., and Mishima, T., "Relationship between solitonic solutions of fivedimensional Einstein equations", Phys. Rev. D, 74, 104004, (2006). Related online version (cited on 14 February 2008):

http://arXiv.org/abs/hep-th/0608169. 5.2.2.4

[239] Tomizawa, S., Morisawa, Y., and Yasui, Y., "Vacuum solutions of five dimensional Einstein equations generated by inverse scattering method", Phys. Rev. D, 73, 064009, (2006). Related online version (cited on 14 February 2008):

http://arXiv.org/abs/hep-th/0512252. 5.2.2.3

[240] Tomizawa, S., and Nozawa, M., "Vaccum solutions of five-dimensional Einstein equations generated by inverse scattering method. II: Production of black ring solution", Phys. Rev. $D, \mathbf{7 3}, 124034,(2006)$. Related online version (cited on 14 February 2008):

http://arXiv.org/abs/hep-th/0604067. 5.2.2.3

[241] Tomizawa, S., Uchida, Y., and Shiromizu, T., "Twist of stationary black hole / ring in five dimensions", Phys. Rev. D, 70, 064020, (2004). Related online version (cited on 14 February 2008):

http://arXiv.org/abs/gr-qc/0405134. 8.4

[242] Tseytlin, A.A., "Extreme dyonic black holes in string theory", Mod. Phys. Lett. A, 11, 689, (1996). Related online version (cited on 14 February 2008):

http://arXiv.org/abs/hep-th/9601177. 7.2.2

[243] Vasudevan, M., Stevens, K.A., and Page, D.N., "Particle motion and scalar field propagation in Myers-Perry black hole spacetimes in all dimensions", Class. Quantum Grav., 22, 1469, (2005). Related online version (cited on 14 February 2008):

http://arXiv.org/abs/gr-qc/0407030. 4.3

[244] Wald, R.M., General Relativity, (University of Chicago Press, Chicago, U.S.A., 1984). 8.6

[245] Walker, M., and Penrose, R., "On Quadratic First Integrals of the Geodesic Equations for Type \{22\} Spacetimes", Commun. Math. Phys., 18, 265-274, (1970). Related online version (cited on 7 July 2008):

http://projecteuclid.org/euclid.cmp/1103842577. 4.3

Living Reviews in Relativity

http: //www . livingreviews . org/lrr-2008-6 
[246] Welch, D.L., "On the smoothness of the horizons of multi - black hole solutions", Phys. Rev. $D, \mathbf{5 2}, 985,(1995)$. Related online version (cited on 14 February 2008): http://arXiv.org/abs/hep-th/9502146. 7.5

[247] Weyl, H., "Zur Gravitationstheorie", Ann. Phys. (Berlin), 54, 117-145, (1917). 5.2.1

[248] Wiseman, T., "Static axisymmetric vacuum solutions and non-uniform black strings", Class. Quantum Grav., 20, 1137, (2003). Related online version (cited on 14 February 2008): http://arXiv.org/abs/hep-th/0209051. 3.4, 6.2

[249] Witten, E., "Anti-de Sitter space and holography", Adv. Theor. Math. Phys., 2, 253, (1998). Related online version (cited on 14 February 2008): http://arXiv.org/abs/hep-th/9802150. 9.2

[250] Witten, E., "Anti-de Sitter space, thermal phase transition, and confinement in gauge theories", Adv. Theor. Math. Phys., 2, 505, (1998). Related online version (cited on 14 February 2008):

http://arXiv.org/abs/hep-th/9803131. 9.2, 9.2, 9.2

[251] Yazadjiev, S.S., "Rotating non-asymptotically flat black rings in charged dilaton gravity", Phys. Rev. D, 72, 104014, (2005). Related online version (cited on 14 February 2008): http://arXiv.org/abs/hep-th/0511016. 7.4

[252] Yazadjiev, S.S., "Completely integrable sector in 5D Einstein-Maxwell gravity and derivation of the dipole black ring solutions", Phys. Rev. D, 73, 104007, (2006). Related online version (cited on 14 February 2008):

http://arXiv.org/abs/hep-th/0602116. 7.4

[253] Yazadjiev, S.S., "Generating dyonic solutions in 5D low-energy string theory and dyonic black rings", Phys. Rev. D, 73, 124032, (2006). Related online version (cited on 14 February 2008): http://arXiv.org/abs/hep-th/0512229. 7.4

[254] Yazadjiev, S.S., "Solution generating in 5D Einstein-Maxwell-dilaton gravity and derivation of dipole black ring solutions", J. High Energy Phys., 2006(07), 036, (2006). Related online version (cited on 14 February 2008):

http://arXiv.org/abs/hep-th/0604140. 7.4

[255] Yazadjiev, S.S., "Black Saturn with dipole ring", Phys. Rev. D, 76, 064011, (2007). Related online version (cited on 14 February 2008):

http://arXiv.org/abs/arXiv:0705.1840. 7.5

[256] Yoo, C.M., Nakao, K.-I., and Ida, D., "Hoop conjecture in five-dimensions: Violation of cosmic censorship", Phys. Rev. D, 71, 104014, (2005). Related online version (cited on 14 February 2008):

http://arXiv.org/abs/gr-qc/0503008. 8.9

[257] Yoshino, H., and Nambu, Y., "High-energy head-on collisions of particles and hoop conjecture", Phys. Rev. D, 66, 065004, (2002). Related online version (cited on 14 February 2008): http://arXiv.org/abs/gr-qc/0204060. 8.9 